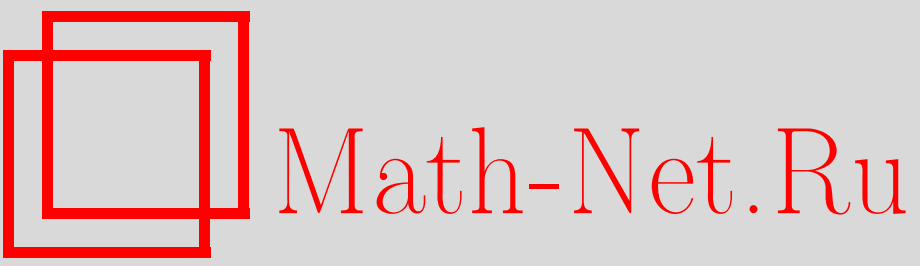

Д. О. Орлов, Производные категории когерентных пучков и эквивалентности между ними, УМН, 2003, том 58, выпуск 3, 89-172

DOI: https://doi.org/10.4213/rm629

Использование Общероссийского математического портала Math-Net.Ru подразумевает, что вы прочитали и согласны с пользовательским соглашением

http://www . mathnet.ru/rus/agreement

Параметры загрузки:

IP : 54.80 .73 .141

26 апреля 2023 г., 05:55:29 


\title{
ПРОИЗВОДНЫЕ КАТЕГОРИИ КОГЕРЕНТНЫХ ПУЧКОВ И ЭКВИВАЛЕНТНОСТИ МЕЖДУ НИМИ
}

\author{
Д. О. Орлов
}

В статье изучаются производные категории когерентных пучков на гладких полных алгебраических многообразиях и эквивалентности между ними. Доказьвается, что каждая эквивалентность представляется объектом на произведении многообразий. Этот резултат применяется для описания абелевых многообразий и К3 поверхностей, имеющих эквивалентные производные категории когерентных пучков.

Библиография: 44 названия.

\section{СОДЕРЖАНИЕ}

Введение

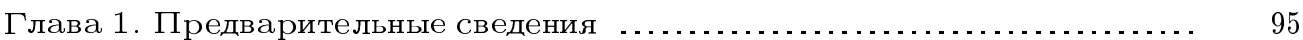

1.1. Триангулированные категории и точные функторы ............... 95

1.2. Производные категории и производные функторы . . . . . . . . . . . . . . . . 101

1.3. Производные категории пучков на схемах ......................... 104

Глава 2. Категории когерентных пучков и функторы между ними ....... 109

2.1. Основные свойства категории когерентных пучков ................. 109

2.2. Примеры эквивалентностей: бирациональные преобразования типа флоп 118

Глава 3. Вполне строгие функторы между производными категориями .. 124

3.1. Диаграммы Постникова и их свертки ............................. 124

3.2. Вполне строгие функторы между производными категориями когерентных пучков . ..................................................... 127

3.3. Построение объекта, представляющего вполне строгий функтор . ..... 129

3.4. Доказателшство основной теоремы ............................ 135

3.5. Приложение: $n$-кошулевость однородной координатной алгебры ....... 144

Глава 4. Производные категории когерентных пучков на К3 поверхнос-

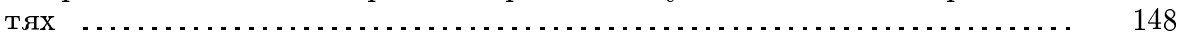

4.1. К3 поверхности и решетка Мукаи . . . . . . . . . . . . . . . . . . . . . . . . . . . 148

4.2. Критерий эквивалентности для производных категорий когерентных пуч-

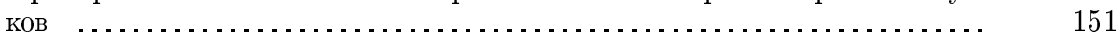

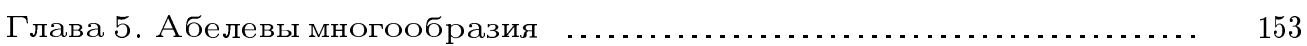

5.1. Эквивалентности между категориями когерентных пучков на абелевых

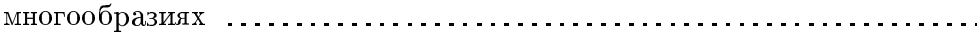

5.2. Объекты, представляющие эквивалентности, и группы автоэквивалент-

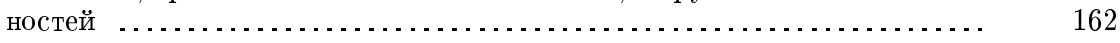

5.3. Полуоднородные векторные расслоения . . . . . . . . . . . . . . . . . . . . . . . 164

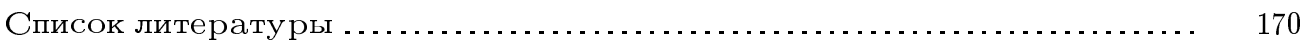




\section{Введение}

Основньми объектами изучения алгебраической геометрии являются алгебраические многообразия (или схемы) и морфизмы между ними. Каждое алгебраическое многообразие $X$ - это окольцованное топологическое пространство и, таким образом, оснашено топологией (чаше всего Зариского) и пучком колец регулярных функций $\mathscr{O}_{X}$.

Изучение алгебраического многообразия - это в большой степени изучение пучков на этом многообразии. И так как пространство окольцовано, то естественными пучками являются пучки $\mathscr{O}_{X}$-модулей, среди которых своей алгебраической природой выделяются квазикогерентные и когерентные пучки. Напомним, что пучок $\mathscr{O}_{X}$-модулей называется квазикогерентньм, если он локально представляется как коядро отображения свободных пучков и назьвается когерентньм, если эти свободные пучки имеют конечный ранг. (Локально свободные пучки на многообразии однозначно соответствуют векторным расслоениям, поэтому в дальнейшем эти термины употребляются как эквивалентные).

Таким образом, с каждым алгебраическим многообразием $X$ связаны абелевы категории (квази)когерентных пучков на нем $\operatorname{coh}(X)$ (и $\mathrm{Q} \operatorname{coh}(X)$ ). Морфизмы между многообразиями индуцируют функторы обратного и прямого образа между этими абелевыми категориями. Однако эти функторы не являются точньми, т.е. не переводят точные последовательности в точные. Данное обстоятельство создает немалые трудности при работе с абелевьми категориями и неточными функторами между ними. Чтобы сохранить функториальность, Картан и Эйленберг [11] ввели понятие производных функторов, которые дают необходимые поправки к неточным функторам. Эта техника была развита Гротендиком в работе [15] и в дальнейшем привела к введению новых понятий: производной категории и производных функторов между ними.

В производных категориях, в отличие от абелевых, нет коротких точных последовательностей и не могут быть определены ядра и коядра морфизмов, но, тем не менее, производные категории обладают некоторой внутренней структурой, которая была оформлена Вердье в понятие триангулированной категории [44].

Переход от абелевых категорий к производным от них позволяет решить многие проблемы, связанные с трудностями при изучении естественных функторов. В качестве одного из первых примеров нужно отметить создание глобальной теории пересечения и доказательство теоремы Римана-Роха. Это было сделано Гротендиком и соавторами в [41] и стало возможным с введением триангулированной категории совершенных комплексов.

Другой пример связан с введением превратных пучков и установлением соответствия Римана-Гильберта между голономными модулями с регулярными особенностями и конструктивньми пучками, которое стало возможным только с привлечением понятия и техники триангулированных категорий (см. [3], [23]).

Многие вопросы, связанные с изучением многообразий, требуют исследования и описания производных категорий когерентных пучков на них. В простейших случаях, когда многообразие - это точка или гладкая кривая, любой объект в производной

Работа вьполнена при частичной поддержке РФФИ (грант № 02-01-00468), Фонда поддержки ведущих научных школ (грант № 00-15-96085) и гранта INTAS-OPEN-2000-269. Исследования, описанные в данной работе, были сделаны при частичной поддержке Американского фонда гражданских исследований (CRDF № RM1-2405-MO-02). Автору также приятно выразить свою благодарность Фонду содействия отечественной науке. 
категории когерентных пучков изоморфен прямой сумме некоторого набора когерентных пучков, взятых с подходяшим сдвигом, то есть каждый $A \in \mathbf{D}^{b}(\operatorname{coh} X)$ изоморфен $\bigoplus_{i=1}^{k} \mathscr{F}_{i}\left[n_{i}\right]$, где $\mathscr{F}_{i}-$ когерентные пучки. Данные примеры отражают тот факт, что гомологическая размерность абелевой категории в этих случаях не превосходит 1. Но для многообразий большей размерности существуют комплексы, которые не изоморфны в производной категории сумме своих когомологий. Поэтому описание производной категории для многообразий размерности больше 1 является трудной и интересной задачей. Первые шаги в этом направлении были сделаны в работах [4] и [2], в которых была описана производная категория когерентных пучков на проективных пространствах, что позволило в дальнейшем применить данную технику к исследованию многообразия модулей векторных расслоений на $\mathbb{P}^{2}$ и $\mathbb{P}^{3}$. В частности, в этих работах было показано, что производная категория когерентных пучков $\mathbf{D}^{b}\left(\operatorname{coh} \mathbb{P}^{n}\right)$ на проективном пространстве эквивалентна производной категории конечномерных модулей над конечномерной алгеброй $A=\operatorname{End}\left(\bigoplus_{i=0}^{i=n} \mathscr{O}(i)\right)$. Этот подход был позднее усовершенствован, и были получены описания производных категорий когерентных пучков на квадриках и на фолаговых многообразиях [20]-[22]).

Введение понятий исключительного набора и полуортогонального разложения позволило сформулировать новые принципы для описания производных категорий когерентных пучков [5], [6]. Оказалось, что наличие полного исключительного набора всегда осуществляет эквивалентность производной категории когерентных пучков с производной категорией конечномерных модулей над конечномерной алгеброй эндоморфизмов данного исключительного набора [5]. Понятие полуортогонального разложения позволило дать описание производной категории раздутия в терминах производных категорий раздуваемого многообразия и подмногообразия, в котором это раздутие происходит [34].

Однако для многих типов многообразий описать производную категорию не представляется возможньм. Тем не менее, естественный вопрос, который возникает при переходе от многообразий к производным категориям когерентных пучков, можно грубо сформулировать так: как много информации сохраняется при данном переходе? На самом деле, выясняется, что это сопоставление сохраняет “почти” всю информацию. Во многих случаях можно даже восстановить само многообразие по его производной категории когерентных пучков - например, если канонический (или антиканонический) пучок многообразия является обильньм [8].

Тем не менее, для некоторых типов многообразий существуют примеры того, когда два разных многообразия имеют эквивалентные производные категории когерентных пучков. Первый пример двух разных многообразий с эквивалентными производными категориями когерентных пучков был найден Мукаи [29]. Он показал, что таковыми являются любое абелево многообразие и двойственное к нему. Эта конструкция была обобщена в работе [38], где для каждого абелева многообразия был введен целый класс абелевых многообразий, имеюших ту же самую производную категорию когерентных пучков.

Данные примеры показывают, что, с одной стороны, существуют многообразия с эквивалентными производными категориями когерентных пучков, а с другой, каждый класс многообразий с эквивалентными производньми категориями когерентных пучков "мал" (во всех примерах он конечен).

Чтобы получить полную классификацию многообразий с эквивалентными произ- 
водньми категориями когерентных пучков, необходимо иметь описание функторов и эквивалентностей между ними. Оказьвается, что эквивалентности всякий раз имеют геометрическую природу, т.е. представляются некоторыми комплексами пучков на произведении.

Поясним, что имеется в виду. Через $\mathbf{D}^{b}(X)$ будем в дальнейшем обозначать ограниченную производную категорию когерентных пучков на $X$. Любой морфизм $f: X \rightarrow Y$ между гладкими полнњми алгебраическими многообразиями индуцирует два точных функтора между ограниченными производными категориями когерентных пучков: функтор прямого образа $\mathbf{R} f_{*}: \mathbf{D}^{b}(X) \longrightarrow \mathbf{D}^{b}(Y)$ и функтор обратного образа $\mathbf{L} f^{*}: \mathbf{D}^{b}(Y) \longrightarrow \mathbf{D}^{b}(X)$, который сопряжен к $\mathbf{R} f_{*}$ слева. Кроме того, каждьй объект $\mathscr{E} \in \mathbf{D}^{b}(X)$ задает точньй функтор тензорного умножения $\stackrel{\mathbf{L}}{\otimes} \mathscr{E}: \mathbf{D}^{b}(X) \longrightarrow \mathbf{D}^{b}(X)$. Используя эти стандартные производные функторы, можно ввести новый большой класс точных функторов между производными категориями $\mathbf{D}^{b}(X)$ и $\mathbf{D}^{b}(Y)$.

Пусть $X$ и $Y$ - два гладких полных многообразия над полем $k$. Рассмотрим декартово произведение $X \times Y$ и обозначим через $p$ и $q$ проекции $X \times Y$ на $X$ и, соответственно, на $Y$

$$
X \stackrel{p}{\longleftarrow} X \times Y \stackrel{q}{\longrightarrow} Y .
$$

Каждый объект $\mathscr{E} \in \mathbf{D}^{b}(X \times Y)$ задает точный функтор $\Phi_{\mathscr{E}}$ из производной категории $\mathbf{D}^{b}(X)$ в производную категорию $\mathbf{D}^{b}(Y)$, которьй определяется по формуле

$$
\Phi_{\mathscr{E}}(\cdot):=\mathbf{R} \cdot q_{*}\left(\mathscr{E} \stackrel{\mathbf{L}}{\otimes} p^{*}(\cdot)\right) .
$$

Каждьй функтор такого вида имеет левый и правьй сопряженные функторы.

Таким образом, каждому гладкому полному алгебраическому многообразию можно сопоставить его производную категорию когерентных пучков, а с каждым объектом $\mathscr{E} \in \mathbf{D}^{b}(X \times Y)$ на произведении двух таких многообразий связать точньй функтор $\Phi_{\mathscr{E}}$ из триангулированной категории $\mathbf{D}^{b}(X)$ в триангулированную категорию $\mathbf{D}^{b}(Y)$. Данная работа посвящена исследованию этого соответствия.

Один из первых вопросов, возникающих при изучении производных категорий когерентных пучков, такой: любой ли функтор между этими категориями представляется объектом на произведении, т.е. имеет вид (1)? В третьей главе дается утвердительньй ответ на этот вопрос в случае, когда функтор является эквивалентностью.

Другими центральными вопросами здесь являются следующие два:

1) Когда производные категории когерентных пучков двух разных гладких полных многообразий эквивалентны как триангулированные категории?

2) Каковагруппа точных автоэквивалентностей производной категории когерентных пучков для данного фиксированного многообразия $X$ ?

Некоторые результаты в этом направлении были уже известны. Существует исчерпывающий ответ на данные вопросы в случае, когда канонический или антиканонический пучок многообразия является обильным. В работе [8] доказано, что гладкое проективное многообразие, канонический (или антиканонический) пучок которого обилен, восстанавливается по своей производной категории когерентных пучков $\mathbf{D}^{b}(X)$. Более того, в этой работе приведена явная конструкция восстановления. Для многообразий данного типа можно также описать и групу точных автоэквивалентностей. 
Теперь опишем содержание и структуру статьи. В статье собраны результаты, большинство из которых в том или ином виде могут быть найдены в работах [7], [8], $[34],[35],[37]$.

В первой главе собраны сведения предварительного характера. Сначала дается определение триангулированной категории и напоминаются понятия точного функтора между триангулированными категориями, локализации триангулированной категории по полной подкатегории и общее определение производного функтора для локализованных триангулированных категорий. Затем вводится определение гомотопической и производной категорий от абелевой категории, а также обсуждаются свойства производных категорий когерентных и квазикогерентных пучков на схемах и функторы между ними.

Во второй главе вводится класс функторов между ограниченными производными категориями когерентных пучков на гладких полных алгебраических многообразиях, которые представляются объектами на произведении, и описьваются их основные свойства. Используя результаты из третьей главы, показывается, что если два гладких проективных многообразия $X$ и $Y$ имеют эквивалентные производные категории, то тогда существует изоморфизм между биградуированными алгебрами $\mathrm{HA}(X)$ и НА $(Y)$, которые определяются по следуюшей формуле:

$$
\mathrm{HA}(X)=\bigoplus_{i, k} \mathrm{HA}_{i, k}(X)=\bigoplus_{i, k} \bigoplus_{p+q=i} \mathrm{H}^{p}\left(X, \Lambda^{q} T_{X} \otimes \omega_{X}^{k}\right)
$$

где $T_{X}$ - касательное, а $\omega_{X}$ - каноническое расслоение на $X$ (теорема 2.1.8 и следствие 2.1.10).

Во втором разделе второй главы приводится цельй класс примеров пары многообразий, имеюших эквивалентные производные категории когерентных пучков. Данные примеры интересны тем, что возникаюшие многообразия бирационально изоморфны (но в общем случае не изоморфны) и связаны преобразованием типа флоп. В частности, данные примеры показьвают, что условие обильности канонического (или антиканонического) класса в теореме о восстановлении не может быть ослаблено.

Пусть $Y$ - гладко вложенное замкнутое подмногообразие в гладком полном алгебраическом многообразии $X$ такое, что $Y \cong \mathbb{P}^{k}$ с нормальным расслоением $N_{X / Y} \cong$ $\mathscr{O}_{Y}(-1)^{\oplus(l+1)}$. Будем предполагать, что $l \leqslant k$. Обозначим через $\widetilde{X}$ раздутие $X$ с центром вдоль $Y$. В этом случае исключительньй дивизор $\widetilde{Y}$ изоморфен произведению проективных пространств $\mathbb{P}^{k} \times \mathbb{P}^{l}$. Сушествует сдутие $\widetilde{X}$ такое, что $\widetilde{Y}$ проектируется на второй сомножитель $\mathbb{P}^{l}$. Рассмотрим диаграмму проекций:

$$
X \stackrel{\pi}{\longleftarrow} \widetilde{\pi^{+}} \underset{\longrightarrow}{\longrightarrow} X^{+}
$$

Бирациональное отображение $f l: X--X^{+}$является простейшим примером преобразования типа фллип-флоп и есть флип при $l<k$ и флоп при $l=k$.

Основная теорема этого раздела связьвает производные категории когерентных пучков на многообразиях $X$ и $X^{+}$. Она говорит, что для любого линейного расслоения $\mathscr{L}$ на $\widetilde{X}$ функтор

$$
\mathbf{R} \pi_{*}\left(\mathbf{L} \pi^{+*}(\cdot) \otimes \mathscr{L}\right): \mathbf{D}^{b}\left(X^{+}\right) \longrightarrow \mathbf{D}^{b}(X)
$$


является вполне строгим, а при $k=l$ этот функтор является эквивалентностью.

Третья глава является центральной. Она посвящена доказательству того факта, что любая эквивалентность между производными категориями когерентных пучков на гладких проективных многообразиях представляется объектом на произведении. Данное утверждение позволяет описьвать эквивалентности между производными категориями когерентных пучков и отвечать на вопрос, когда два разных многообразия имеют эквивалентные производные категории когерентных пучков.

На самом деле в этой главе доказьвается более общий факт: а именно, что любой функтор между ограниченными производньми категориями когерентных пучков нагладких проективных многообразиях, который является вполне строгим и имеет сопряженньй, может быть представлен объектом на произведении этих многообразий, т.е. изоморфен функтору $\Phi_{\mathscr{E}}$, определенному по правилу (1). Более того, представляющий объект однозначно определен с точностью до изоморфизма (теорема 3.2.1).

В четвертой главе изучаются производные категории когерентных пучков на К3 поверхностях. Для любой К3 поверхности $S$ на решетке когомологий $\mathrm{H}^{*}(S, \mathbb{Z})$ можно определить симметрическую билинейную форму по правилу

$$
\left(u, u^{\prime}\right)=r \cdot s^{\prime}+s \cdot r^{\prime}-\alpha \cdot \alpha^{\prime} \in \mathrm{H}^{4}(S, \mathbb{Z}) \cong \mathbb{Z}
$$

для каждой пары $u=(r, \alpha, s), u^{\prime}=\left(r^{\prime}, \alpha^{\prime}, s^{\prime}\right) \in \mathrm{H}^{0}(S, \mathbb{Z}) \oplus \mathrm{H}^{2}(S, \mathbb{Z}) \oplus \mathrm{H}^{4}(S, \mathbb{Z})$. Решетка когомологий $\mathrm{H}^{*}(S, \mathbb{Z})$ с этой билинейной формой $(\cdot, \cdot)$ называется решеткой Мукаи и обозначается через $\widetilde{\mathrm{H}}(S, \mathbb{Z})$.

На решетке $\widetilde{\mathrm{H}}(S, \mathbb{Z})$ имеется естественная структура Ходжа. В данном случае, говоря о структуре Ходжа, мы имеем в виду то, что в пространстве $\widetilde{\mathrm{H}}(S, \mathbb{C})$ зафиксировано одномерное подпространство $\mathrm{H}^{2,0}(S)$. Назовем решетки Мукаи двух К3 поверхностей ходжево изометричными, если сушествует изометрия между ними, которая переводит одномерное подпространство $\mathrm{H}^{2,0}\left(S_{1}\right)$ в $\mathrm{H}^{2,0}\left(S_{2}\right)$.

Главная теорема этой главы (теорема 4.2.1) говорит, что производные категории когерентных пучков $\mathbf{D}^{b}\left(S_{1}\right)$ и $\mathbf{D}^{b}\left(S_{2}\right)$ двух К3 поверхностей над полем $\mathbb{C}$ эквивалентны как триангулированные категории тогда и только тогда, когда сушествует ходжева изометрия $f: \widetilde{\mathrm{H}}\left(S_{1}, \mathbb{Z}\right) \stackrel{\sim}{\longrightarrow} \widetilde{\mathrm{H}}\left(S_{2}, \mathbb{Z}\right)$ между их решетками Мукаи. Эта теорема имеет и другой вариант в терминах решеток трансцедентных циклов (теорема 4.2.4).

Учитывая теорему типа Торелли для К3 поверхностей [39], [27], которая говорит, что К3 поверхность восстанавливается по своей структуре Ходжа на вторых когомологиях, получаем ответ в терминах структур Ходжа на вопрос, когда производные категории когерентных пучков двух К3 поверхностей эквивалентны.

В пятой главе изучаются производные категории когерентных пучков на абелевых многообразиях и их группы автоэквивалентностей.

Пусть $A$ - абелево многообразие и $\widehat{A}$ - двойственное абелево многообразие. В работе [29] было доказано, что производные категории когерентных пучков $\mathbf{D}^{b}(A)$ и $\mathbf{D}^{b}(\widehat{A})$ эквивалентны, и эквивалентность, которая назьвается преобразованием Фурье-Мукаи, может быть задана с помошью линейного расслоения Пуанкаре $\mathscr{P}_{A}$ на произведении $A \times \widehat{A}$ по правилу (1): $F \mapsto \mathbf{R}^{*} p_{2 *}\left(\mathscr{P}_{A} \otimes p_{1}^{*}(F)\right)$.

Эта конструкция Мукаи была обобщена в статье [38] следующим образом. Рассмотрим два абелевых многообразия $A$ и $B$ и изоморфизм $f$ между абелевыми многообразиями $A \times \widehat{A}$ и $B \times \widehat{B}$. Запишем $f$ в матричном виде

$$
f=\left(\begin{array}{cc}
x & y \\
z & w
\end{array}\right)
$$


где $x$ - гомоморфизм из $A$ в $B, y$ - из $\widehat{A}$ в $B$, и так далее. Назовем его изометричным, если обратньй к $f$ имеет следующий вид:

$$
f^{-1}=\left(\begin{array}{cc}
\widehat{w} & -\widehat{y} \\
-\widehat{z} & \widehat{x}
\end{array}\right) .
$$

Определим множество $U(A \times \widehat{A}, B \times \widehat{B})$ как множество изометричных изоморфизмов $f$. Если $B=A$, то это множество будем обозначать $U(A \times \widehat{A})$. Отметим, что $U(A \times \widehat{A})$ является подгруппой в $\operatorname{Aut}(A \times \widehat{A})$.

В статье [38] доказьвается, что если для двух абелевых многообразий над алгебраически замкнутым полем $A$ и $B$ сушествует изометричный изоморфизм между $A \times \widehat{A}$ и $B \times \widehat{B}$, то производные категории когерентных пучков $\mathbf{D}^{b}(A)$ и $\mathbf{D}^{b}(B)$ эквивалентны.

В этой главе доказьвается равносильность этих условий над алгебраически замкнутьм полем характеристики 0 , т.е. производные категории $\mathbf{D}^{b}(A)$ и $\mathbf{D}^{b}(B)$ эквивалентны тогда и только тогда, когда сушествует изометричньй изоморфизм из $A \times \widehat{A}$ в $B \times \widehat{B}$. На самом деле, в одну сторону это утверждение верно над произвольным полем (теорема 5.1.16). Как следствие получаем, что существует только конечное число неизоморфных абелевых многообразий, производные категории которых эквивалентны $\mathbf{D}^{b}(A)$ для заданного абелева многообразия $A$ (следствие 5.1.17).

Представляя эквивалентности объектами на произведении, мы строим отображение из множества всех точных эквивалентностей между $\mathbf{D}^{b}(A)$ и $\mathbf{D}^{b}(B)$ в множество изометричных изоморфизмов из $A \times \widehat{A}$ в $B \times \widehat{B}$. Далее показьвается, что это отображение функториально (предложение 5.1.12). В частности, получается гомоморфизм из группы точных автоэквивалентностей категории $\mathbf{D}^{b}(A)$ в группу $U(A \times \widehat{A})$ изометричных автоморфизмов многообразия $A \times \widehat{A}$.

В разделе 5.2 описьвается ядро этого гомоморфизма, которое оказьвается изоморфно прямой сумме $\mathbb{Z}$ и групшы $k$-точек многообразия $A \times \widehat{A}$ (предложение 5.2 .3 ). Технически это описание опирается на тот факт, что объект на произведении абелевых многообразий, задаюший эквивалентность, на самом деле является пучком с точностью до сдвига в производной категории (предложение 5.2.2).

В последнем разделе 5.3 в предположении, что основное поле алгебраически замкнуто и $\operatorname{char}(k)=0$, приводится другое доказательство утверждения из [38], основанное на фактах из статьи [30], в которой описьваются полуоднородные расслоения на абелевых многообразиях. В частности, получается описание для групшы автоэквивалентностей в виде точной последовательности

$$
0 \longrightarrow \mathbb{Z} \oplus(A \times \widehat{A})_{k} \longrightarrow \text { Auteq } \mathbf{D}^{b}(A) \longrightarrow U(A \times \widehat{A}) \longrightarrow 1 .
$$

\section{Глава 1. Предварительные сведения}

1.1. Триангулированные категории и точные функторы. Подробное изложение фактов, собранных в этой главе, может быть найдено в работах [14], [24], [25], [44]. Понятие триангулированной категории было впервые введено Верде в работе [44]. Пусть $\mathscr{D}$ - некоторая аддитивная категория. Структура триангулированной

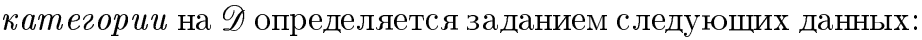

а) аддитивный функтор сдвига $[1]: \mathscr{D} \longrightarrow \mathscr{D}$, который является автоэквивалентностью; 
b) некоторьй класс выделенных треугольников:

$$
X \stackrel{u}{\longrightarrow} Y \stackrel{v}{\longrightarrow} Z \stackrel{w}{\longrightarrow} X[1]
$$

которые должны удовлетворять набору аксиом Т1-Т4.

T1. а) Для каждого объекта $X$ треугольник $X \stackrel{\text { id }}{\longrightarrow} X \longrightarrow 0 \longrightarrow X[1]$ выделен.

b) Если треугольник выделен, то любой изоморфный ему также выделен.

c) Любой морфизм $X \stackrel{u}{\longrightarrow} Y$ можно дополнить до выделенного треугольника $X \stackrel{u}{\longrightarrow} Y \stackrel{v}{\longrightarrow} Z \stackrel{w}{\longrightarrow} X[1]$.

Т2. Треугольник $X \stackrel{u}{\longrightarrow} Y \stackrel{v}{\longrightarrow} Z \stackrel{w}{\longrightarrow} X[1]$ выделен тогда и только тогда, когда выделен треугольник $Y \stackrel{v}{\longrightarrow} Z \stackrel{w}{\longrightarrow} X[1] \stackrel{-u[1]}{\longrightarrow} Y[1]$.

Т3. Если даны два выделенных треугольника и два морфизма между их началами, образующие коммутативный квадрат, тогда эта диаграмма дополняется до морфизма треугольников:

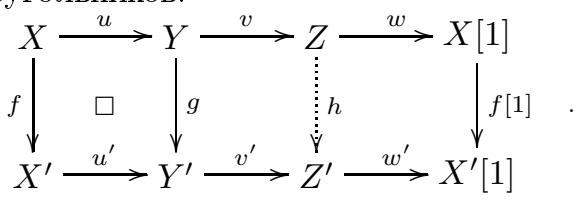

Т4. Для каждой пары морфизмов $X \stackrel{u}{\longrightarrow} Y \stackrel{v}{\longrightarrow} Z$ существует коммутативная диаграмма

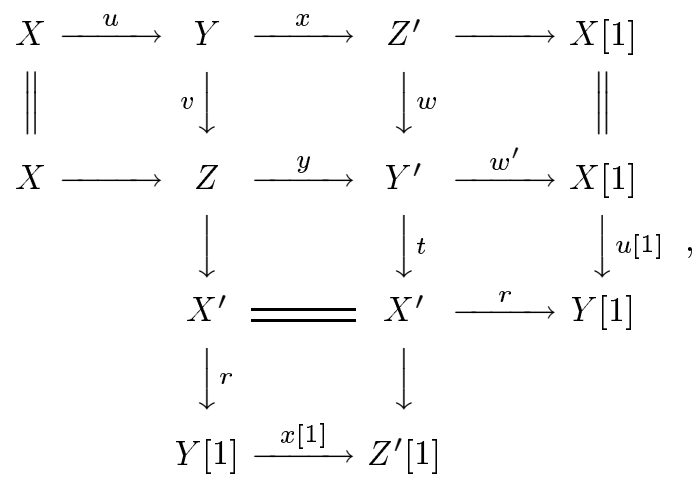

где первые две строчки и два центральных столбца - выделенные треугольники.

Пусть $\mathscr{D}$-триангулированная категория. Полная аддитивная подкатегория $\mathscr{N} \subset \mathscr{D}$ называется триангулированной подкатегорией, если она замкнута относительно функтора сдвига и взятия конуса морфизма, т.е. если два объекта какого-нибудь треугольника

$$
X \longrightarrow Y \longrightarrow Z \longrightarrow X[1]
$$

принадлежат подкатегории $\mathcal{N}$, то и третий также принадлежит $\mathscr{N}$. Теперь опишем функторы между триангулированными категориями, которые имеет смысл рассматривать. 
ОПРЕДЕЛЕНИЕ 1.1.1. Аддитивньй функтор $F: \mathscr{D} \longrightarrow \mathscr{D}^{\prime}$ между двумя триангулированными категориями $\mathscr{D}$ и $\mathscr{D}^{\prime}$ называется точнылм, если он

а) коммутирует с функторами сдвига, т.е. зафиксирован изоморфизм функторов:

$$
t_{F}: F \circ[1] \stackrel{\sim}{\longrightarrow}[1] \circ F
$$

b) переводит каждый выделенньй треугольник из $\mathscr{D}$ в выделенньй треугольник в $\mathscr{D}^{\prime}$ (используя изоморфизм $t_{F}$, мы $F(X[1])$ заменяем на $\left.F(X)[1]\right)$.

Из определения сразу следует, что композиция двух точных функторов также является точным функтором. Другое свойство, которое будет нам необходимо, касается сопряженных функторов.

Лемма 1.1.2 [6], [8]. Если функтор $G: \mathscr{D}^{\prime} \longrightarrow \mathscr{D}$ - левый (или правый) сопряженный к точному функтору $F: \mathscr{D} \longrightarrow \mathscr{D}^{\prime}$, тогда функтор $G$ также является точныц.

Дадим определение и опишем основные свойства функтора Серра, абстрактное определение которого было дано в статье [6] (см. также [8]).

ОПРЕДЕЛЕНИЕ 1.1.3. Пусть $\mathscr{D}-k$-линейная категория с конечномерными пространствами Hom между объектами. Ковариантный функтор $S: \mathscr{D} \rightarrow \mathscr{D}$ назьвается функтором Серра, если он является эквивалентностью и существует бифункториальньй изоморфизм

$$
\varphi_{A, B}: \operatorname{Hom}_{\mathscr{D}}(A, B) \stackrel{\sim}{\longrightarrow} \operatorname{Hom}_{\mathscr{D}}(B, S A)^{*}
$$

для любых объектов $A, B \in \mathscr{D}$.

ЛЕмма 1.1.4 [8]. Всякая әквивалентность $\Phi: \mathscr{D} \longrightarrow \mathscr{D}^{\prime}$ коммутирует с функторами Серра, т.е. существует естественный изоморфизм функторов $\Phi$ ○ $\stackrel{\sim}{\rightarrow}$ $S^{\prime} \circ \Phi$, где $S$ и $S^{\prime}-\oint у н к т о р ы ~ С е р р а ~ в ~ к а т е г о р и я х ~ \mathscr{D ~ и ~} \mathscr{D}^{\prime}$ соответственно.

Если у нас имеются два функтора Серра в одной категории, то они изоморфны, и этот изоморфизм коммутирует с бифункториальными изоморфизмами $\varphi A, B$ в определении функтора Серра. Действительно, пусть $S$ и $S^{\prime}-$ два функтора Серра в категории $\mathscr{D}$. Тогда для любого объекта $A$ из $\mathscr{D}$ имеется естественньй изоморфизм:

$$
\operatorname{Hom}(A, A) \cong \operatorname{Hom}(A, S A)^{*} \cong \operatorname{Hom}\left(S A, S^{\prime} A\right)
$$

Рассматривая образ единичного морфизма $\mathrm{id}_{A}$ относительно этого отождествления, получаем морфизм $S A \longrightarrow S^{\prime} A$, которьй и дает изоморфизм $S \stackrel{\sim}{\rightarrow} S^{\prime}$.

Таким образом, функтор Серра в категории $\mathscr{D}$, если он существует, однозначно определен (с точностью до изоморфизма). Нас в дальнейшем будет интересовать функтор Серра в триангулированных категориях. 
Лемма 1.1.5 [6]. Функтор Серра в триангулированной категории является точныц.м.

Напомним определение локализации категории и, в частности, локализации триангулированной категории по полной триангулированной подкатегории (см. [13]).

Пусть $\mathscr{C}$ - категория и $\Sigma$ - некоторый класс морфизмов в $\mathscr{C}$. Локализация категории по классу морфизмов $\Sigma$ имеет хорошее описание, если $\Sigma$ допускает исчисление левых частных, т.е. выполняются следуюшие свойства:

L1. Bсе тождественные морфизмы категории принадлежат $\Sigma$.

L2. Композиция любых двух морфизмов из $\Sigma$ также принадлежит $\Sigma$.

L3. Любую диаграмму вида $X^{\prime} \stackrel{s}{\longleftarrow} X \stackrel{u}{\longleftrightarrow} Y$, где $s \in \Sigma$, можно дополнить до коммутативного квадрата

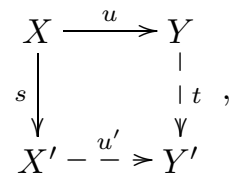

где $t \in \Sigma$.

L4. Если морфизмы $f, g$ и морфизм $s \in \Sigma$ обладают тем свойством, что $f s=g s$, то существует $t \in \Sigma$ такой, что $t f=t g$.

Если $\Sigma$ допускает исчисление частных, то категорию $\mathscr{C}\left[\Sigma^{-1}\right]$ можно описать следуюшим способом. Объекты $\mathscr{C}\left[\Sigma^{-1}\right]$ те же, что и объекты $\mathscr{C}$. Морфизмы из $X$ в $Y-$ это классы эквивалентности диаграмм $(s, f)$ в $\mathscr{C}$ вида

$$
X \stackrel{f}{\longrightarrow} Y^{\prime} \stackrel{s}{\longleftarrow} Y, \quad s \in \Sigma,
$$

причем две диаграммы $(f, s)$ и $(g, t)$ эквивалентны, если их можно включить в коммутативную диаграмму

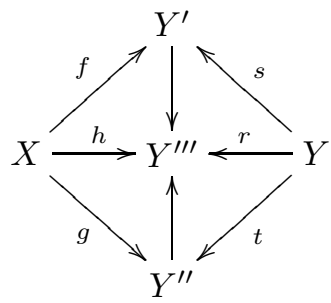

такую, что $r \in \Sigma$.

Композиция морфизмов $(f, s)$ и $(g, t)$ есть морфизм $\left(g^{\prime} f, s^{\prime} t\right)$, которьй строится с помощью квадрата из L3:

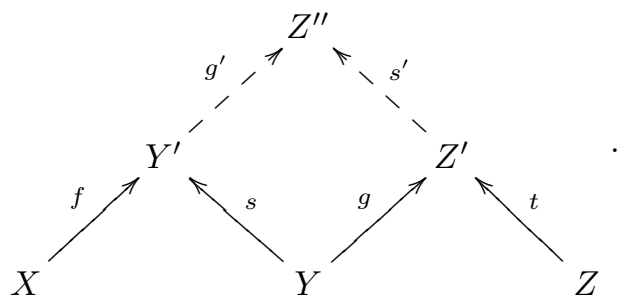

Нетрудно видеть, что $\mathscr{C}\left[\Sigma^{-1}\right]$, построенная таким образом, действительно является категорией (ее объекты образуют множество), а канонический функтор

$$
Q: \mathscr{C} \longrightarrow \mathscr{C}\left[\Sigma^{-1}\right], \quad X \mapsto X, \quad f \mapsto(f, 1),
$$


обрашает все морфизмы из $\Sigma$ и является универсальньм в этом смысле (см. [13]).

Рассмотрим полную подкатегорию $\mathscr{B} \in \mathscr{C}$ и обозначим через $\Sigma \cap \mathscr{B}$ класс морфизмов в $\mathscr{B}$, также принадлежаших $\Sigma$. Будем говорить, что $\mathscr{B}$ кофинальна справа в $\mathscr{C}$ относительно $\Sigma$, если для каждого $s: X \longrightarrow X^{\prime}$ в $\Sigma$ с $X \in \mathscr{B}$ найдется морфизм $f: X^{\prime} \longrightarrow Y$ такой, что $f s \in \Sigma \cap \mathscr{B}$.

Лемма 1.1.6 [17], [25]. Класс $\Sigma \cap \mathscr{B}$ также допускает исчисление левых частных, и если $\mathscr{B}$ является кофинальной справа в $\mathscr{C}$ относительно $\Sigma$, то канонический функтор

$$
\mathscr{B}\left[(\Sigma \cap \mathscr{B})^{-1}\right] \longrightarrow \mathscr{C}\left[\Sigma^{-1}\right]
$$

является вполне строгим.

Напомним определение вполне строгого функтора.

ОПРЕДЕЛЕнИЕ 1.3. Функтор $F: \mathscr{C} \longrightarrow \mathscr{D}$ назьвается вполне строгим, если для любых двух объектов $X, Y \in \mathscr{C}$ естественное отображение

$$
\operatorname{Hom}(X, Y) \longrightarrow \operatorname{Hom}(F X, F Y)
$$

является биекцией.

Пусть теперь $\mathscr{D}$ - триангулированная категория и $\mathscr{N}$ - полная триангулированная подкатегория. Обозначим через $\Sigma$ класс морфизмов $s$ в $\mathscr{D}$, которые включаются в точньй треугольник

$$
N \longrightarrow X \stackrel{s}{\longrightarrow} X^{\prime} \longrightarrow N[1]
$$

где $N \in \mathcal{N}$, и назовем $\Sigma$ мультипликативной системой, ассоциированной с подкатегорий $\mathscr{N}$. Из общей теории локализации следует сушествование аддитивной категории $\mathscr{D}\left[\Sigma^{-1}\right]$ и аддитивного функтора локализации $Q: \mathscr{D} \longrightarrow \mathscr{D}\left[\Sigma^{-1}\right]$. Можно оснастить категорию $\mathscr{D}\left[\Sigma^{-1}\right]$ функтором сдвига, индуцированным функтором $[1]: \mathscr{D} \longrightarrow \mathscr{D}$. Кроме того, определим выделенные треугольники в $\mathscr{D}\left[\Sigma^{-1}\right]$ как треугольники, изоморфные образу выделенных треугольников из $\mathscr{D}$ при локализации. Положим

$$
\mathscr{D} / \mathscr{N}:=\mathscr{D}\left[\Sigma^{-1}\right]
$$

ПРЕДЛОЖЕНИЕ 1.1.8. Категория $\mathscr{D} / \mathscr{N}$, оснащенная описанной выще структурой, становится триангулированной категорией, а функтор $Q: \mathscr{D} \longrightarrow \mathscr{D} / \mathscr{N}$ становится триангулированным функтором.

Отметим, что в данной ситуации система $\Sigma$ допускает исчисление левых (и правых) частных, поэтому у категории $\mathscr{D} / \mathscr{N}$ сушествует хорошее описание, которое было приведено вьше. Теперь, следуя Делиню [12] (см. также [25]), опишем общую конструкцию производных функторов для локализации триангулированных категорий. Пусть $\mathscr{C}$ и $\mathscr{D}$ - триангулированные категории и $F: \mathscr{C} \longrightarrow \mathscr{D}$ - точный функтор. Пусть $\mathscr{M} \subset \mathscr{C}$ и $\mathscr{N} \subset \mathscr{D}$ - полные триангулированные категории. Так как не предполагается, что $F \mathscr{M} \subset \mathscr{N}$, то функтор $F$ не индуцирует никакого функтора из $\mathscr{C} / \mathscr{M}$ в $\mathscr{D} / \mathscr{N}$. Однако может существовать некоторое каноническое приближение к индуцированному функтору, а именно точньй функтор $\mathbf{R} F: \mathscr{C} / \mathscr{M} \longrightarrow \mathscr{D} / \mathscr{N}$ и морфизм точных функторов сап: $Q F \longrightarrow(\mathbf{R} F) Q$. Построение происходит следуюшим образом. Обозначим 
через $\Sigma$ мультипликативную систему, ассоциированную с подкатегорией $\mathscr{M}$. Пусть $Y$ - объект $\mathscr{C} / \mathscr{M}$. Определим контравариантный функтор $\mathbf{r} F Y$ из $\mathscr{D} / \mathscr{N}$ в категорию абелевых групп по следуюшему правилу: значение $\mathbf{r} F Y(X)$ на объекте $X \in \mathscr{D} / \mathscr{N}$ это классы эквивалентностей пар $(s, f)$,

$$
Y \stackrel{s}{\longrightarrow} Y^{\prime}, \quad X \stackrel{f}{\longrightarrow} F Y^{\prime}
$$

где $s \in \Sigma$ и $f$-морфизм в $\mathscr{D} / \mathscr{N}$. Две такие пары $(s, f)$ и $(t, g)$ эквивалентны, если сушествуют коммутативные диаграммы в $\mathscr{C}$ и $\mathscr{D} / \mathscr{N}$
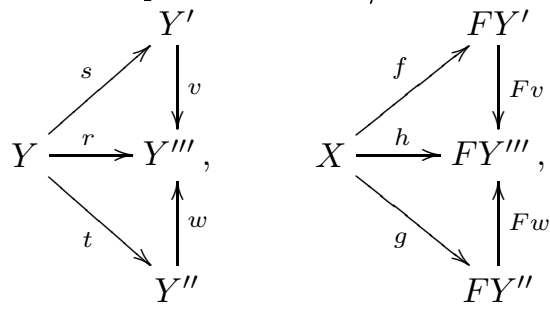

где $r \in \Sigma$. Если функтор $\mathbf{r} F Y$ представим, то определим $\mathbf{R} F Y$ как объект, представляюший этот функтор, и будем говорить, что правый производньй функтор $\mathbf{R} F$ определен на $Y$. В этом случае имеем изоморфизм

$$
\operatorname{Hom}(X, \mathbf{R} F Y) \cong \mathbf{r} F Y(X)
$$

Нетрудно проверить, что для любого морфизма $\alpha: Y \longrightarrow Z$ в $\mathscr{C} / \mathscr{M}$ определен морфизм функторов $\mathbf{r} F \alpha: \mathbf{r} F Y \longrightarrow \mathbf{r} F Z$. Если теперь производньй функтор $\mathbf{R} F$ определен на обоих объектах $Y$ и $Z$, то определен и морфизм $\mathbf{R} F \alpha$. Таким образом, $\mathbf{R} F$ становится функтором $\mathscr{W} \longrightarrow \mathscr{D} / \mathscr{N}$ на некоторой полной подкатегории $\mathscr{W} \subset \mathscr{C} / \mathscr{M}$, состоящей из объектов, на которых $\mathbf{R} F$ определен.

ПРЕДЛОЖЕНИЕ $1.1 .9[12]$. Если

$$
X \longrightarrow Y \longrightarrow Z \longrightarrow X[1]
$$

- выделенный треугольник в $\mathscr{C} / \mathscr{M}$ и $\mathbf{R} F$ определен на $X$ и $Z$, то он определен также на $Y$ и переводит данный треугольник в выделенный треугольник в $\mathscr{D} / \mathscr{N}$. Таким образом, W - триангулированная подкатегория в $\mathscr{C} / \mathscr{M}$ и $\mathbf{R} F: \mathscr{W} \longrightarrow \mathscr{D} / \mathscr{N}-$ точный функтор.

Из построения производного функтора для любого объекта $Y \in \mathscr{C}$ непосредственно следует существование канонического морфизма сап: $Q F Y \longrightarrow(\mathbf{R} F) Q Y$, если, конечно, $\mathbf{R} F$ определен на объекте $Q Y \in \mathscr{C} / \mathscr{M}$. Все такие морфизмы задают естественное преобразование триангулированных функторов сап: $\left.\left.Q F\right|_{\mathscr{W}} \longrightarrow(\mathbf{R} F) Q\right|_{\mathscr{W}}$.

Левьй производньй функтор $\mathbf{L} F$ определяется двойственньм образом: для $Y \in$ $\mathscr{C} / \mathscr{M}$ определяем ковариантньй функтор $1 F Y$, значение которого на $X \in \mathscr{D} / \mathscr{N}$ есть классы эквивалентностей пар $(s, f)$,

$$
Y^{\prime} \stackrel{s}{\longrightarrow} Y, \quad F X^{\prime} \stackrel{f}{\longrightarrow} Y
$$


где $s \in \Sigma$ и $f$-морфизм в $\mathscr{D} / \mathscr{N}$. Тогда объект $\mathbf{L} F Y$, если он сушествует, представляет функтор $\mathbf{l} F Y$, т.е. $\operatorname{Hom}(\mathbf{L} F Y, X) \cong 1 F Y(X)$. Существует канонический морфизм can: $\mathbf{L} F Q Y \longrightarrow Q F Y$.

Предположим, что функтор $F: \mathscr{C} \longrightarrow \mathscr{D}$ такой, что он переводит подкатегорию $\mathscr{M}$ в $\mathscr{N}$. В этом случае производные функторы $\mathbf{R} F$ и $\mathbf{L} F$ оба изоморфны каноническому функтору $\mathscr{C} / \mathscr{M} \longrightarrow \mathscr{D} / \mathscr{N}$, которьй индуцирован функтором $F$.

Пусть $j: \mathscr{V} \hookrightarrow \mathscr{C}$ - вложение полной триангулированной подкатегории, которая кофинальна справа относительно $\Sigma$. По лемме 1.1.6 индуцированньй функтор $\mathscr{V} /(\mathscr{V} \cap \mathscr{M}) \longrightarrow \mathscr{C} / \mathscr{M}$, которьй обозначим через $\mathbf{R} j$, является вполне строгим.

Лемма 1.1.10. Для каждого обвекта $V \in \mathscr{V}$ функтор $\mathbf{R} F$ определен на $V$ тогда и только тогда, когда $\mathbf{R}(F j)$ определен на $V$, и имеется изоморфизм функторов $\mathbf{R}(F j) V \stackrel{\sim}{\longrightarrow} \mathbf{R} F \mathbf{R} j V$.

Теперь опишем условия, при которых правьй производный функтор определен на всей категории $\mathscr{C}$.

ОПРЕДЕЛЕНИЕ 1.1.11. Объект $Y \in \mathscr{C}$ назьвается $F$-расщепимым (справа) относительно $\mathscr{M}$ и $\mathscr{N}$, если $\mathbf{R} F$ определен на $Y$ и канонический морфизм $Q F Y \longrightarrow$ $(\mathbf{R} F) Q Y$ является изоморфизмом.

Следующая лемма дает характеризацию $F$-расщепимых объектов.

Лемма 1.1.12. Облект $Y \in \mathscr{C}$ является F-расщепимым тогда и только тогда, когда для каждого морфизма $s: Y \longrightarrow Y^{\prime}$ из $\Sigma$, морфизм $Q F s$ допускает ретракцию, т.е. существует $p: Q F Y^{\prime} \longrightarrow Q F Y$ такой, что $p \circ Q F s=\mathrm{id}$.

Будем говорить, что $\mathscr{C}$ имеет достаточно много F-расщепимых обвектов (относительно $\mathscr{M}$ и $\mathscr{N})$, если для каждого $Y \in \mathscr{C}$ сушествует морфизм $s: Y \longrightarrow Y_{0}$ из $\Sigma$ такой, что $Y_{0}$ является $F$-расщепимым. В этом случае $\mathbf{R} F$ определен на всей категории $\mathscr{C} / \mathscr{M}$ и имеются изоморфизмы

$$
\mathbf{R} F Y \stackrel{\sim}{\longrightarrow} \mathbf{R} F Y_{0} \stackrel{\sim}{\sim} F Y_{0} .
$$

В заключение этого раздела скажем несколько слов о сопряженных функторах. Предположим, что сушествует левый сопряженный к $F$ функтор $G: \mathscr{D} \longrightarrow \mathscr{C}$. Допустим, существуют (т.е. везде определены) производные функторы $\mathbf{R} F$ и $\mathbf{L} G$. Тогда функтор $\mathbf{L} G$ также является левым сопряженным к $\mathbf{R} F$, и, значит, имеется функториальный изоморфизм

$$
\operatorname{Hom}(\mathbf{L} G X, Y) \cong \operatorname{Hom}(X, \mathbf{R} F Y),
$$

где $X \in \mathscr{D} / \mathscr{N}$ и $Y \in \mathscr{C} / \mathscr{M}$.

1.2. Производные категории и производные функторы. Пусть $\mathscr{A}$ - аддитивная категория. Обозначим через $\mathbf{C}(\mathscr{A})$ категорию дифференциальных комплексов. Объекты этой категории - это комплексы

$$
M^{\cdot}=\left(\cdots \longrightarrow M^{p} \stackrel{d^{p}}{\longrightarrow} M^{p+1} \longrightarrow \cdots\right), \quad M^{p} \in \mathscr{A}, \quad p \in \mathbb{Z}, \quad d^{2}=0,
$$

а морфизмы $f: M^{\cdot} \longrightarrow N^{\cdot}-$ это наборы морфизмов $f^{p}: M^{p} \longrightarrow N^{p}$ в категории $\mathscr{A}$, которые перестановочны с дифференциалами, т.е.

$$
d_{N} f^{p}-f^{p+1} d_{M}=0 \text { для всех } p .
$$


Будем обозначать через $\mathbf{C}^{+}(\mathscr{A}), \mathbf{C}^{-}(\mathscr{A}), \mathbf{C}^{b}(\mathscr{A})$ полные подкатегории в $\mathbf{C}(\mathscr{A})$, образованные комплексами $M^{\cdot}$, для которых $M^{p}=0$, соответственно, для всех $p \ll 0$, для всех $p \gg 0$, для всех $p \gg 0$ и всех $p \ll 0$.

Морфизм комплексов $f: M^{\cdot} \longrightarrow N^{*}$ будем назьвать гомотопным нулю, если $f^{p}=$ $d_{N} h^{p}+h^{p+1} d_{M}$ для всех $p \in \mathbb{Z}$ для некоторого семейства морфизмов $h^{p}: M^{p+1} \longrightarrow$ $N^{p}$. Определим гомотопическую категорию $\mathbf{H}(\mathscr{A})$ как категорию, которая имеет те же самые объекты, что и $\mathbf{C}(\mathscr{A})$, а морфизмы в $\mathbf{H}(\mathscr{A})$ - это классы $\bar{f}$ морфизмов между комплексами $f$ по модулю морфизмов, гомотопных нулю.

Зададим функтор сдвига $[1]: \mathbf{H}(\mathscr{A}) \longrightarrow \mathbf{H}(\mathscr{A})$ по правилу

$$
(M[1])^{p}=M^{p+1}, \quad d_{M[1]}=-d_{M} .
$$

Определим стандартньй треугольник в $\mathbf{H}(\mathscr{A})$ как последовательность

$$
L \stackrel{\bar{f}}{\longrightarrow} M \stackrel{\bar{g}}{\longrightarrow} C f \stackrel{\bar{h}}{\longrightarrow} L[1]
$$

где $f: L \longrightarrow M-$ некоторьй морфизм комплексов, $C f=M \oplus L[1]-$ градуированное векторное пространство с дифференциалом

$$
d_{C f}=\left(\begin{array}{cc}
d_{M} & f \\
0 & -d_{L}
\end{array}\right),
$$

морфизм $g$ - каноническое вложение $M \longrightarrow C f$ и $-h$ - каноническая проекция. Как обычно, $C f$ назьвается конусом морфизма $f$.

ЛЕмма 1.2.1. Категория $\mathbf{H}(\mathscr{A})$, снабжсенная функтором сдвига [1] и классом треугольников, изоморфных стандартньм, является триангулированной категорией.

Обозначим через $\mathbf{H}^{+}(\mathscr{A}), \mathbf{H}^{-}(\mathscr{A})$ и $\mathbf{H}^{b}(\mathscr{A})$ образы категорий $\mathbf{C}^{+}(\mathscr{A}), \mathbf{C}^{-}(\mathscr{A})$ и $\mathbf{C}^{b}(\mathscr{A})$ соответственно в категории $\mathbf{H}(\mathscr{A})$. Эти категории также являются триангулированными. Пусть теперь $\mathscr{A}$ - это абелева категория. Для определения производной категории от абелевой категории необходимо напомнить понятия ацикличного комплекса и квазиизоморфизма. Для любого комплекса $N^{*}$ и каждого $p \in \mathbb{Z}$ определены его когомологии $H^{p}\left(N^{*}\right) \in \mathscr{A}$, которые суть $\operatorname{Ker} d^{p} / \operatorname{Im} d^{p-1}$. Таким образом, для каждого целого $p$ у нас имеется аддитивный функтор $H^{p}: \mathbf{C}(\mathscr{A}) \longrightarrow \mathscr{A}$, которьй сопоставляет комплексу $N^{\cdot}$ его $p$-ю когомологию $H^{p}\left(N^{\bullet}\right) \in \mathscr{A}$.

Комплекс $N^{*} \in \mathbf{C}(\mathscr{A})$ называется ацикличным в $n$-м члене, если $H^{n}\left(N^{*}\right)=0$, и называется просто ацикличным, если все его когомологии $H^{n}\left(N^{*}\right), n \in \mathbb{Z}$ нулевые.

Обозначим через $\mathcal{N}$ полную подкатегорию в $\mathbf{H}(\mathscr{A})$, состоящую из всех ацикличных комплексов. Следующая лемма практически очевидна.

ЛЕмма 1.2.2. Подкатегория $\mathscr{N}$ является полной триангулированной подкатегорией в $\mathbf{H}(\mathscr{A})$.

Морфизм $f: X \longrightarrow Y$ в категории $\mathbf{H}(\mathscr{A})$ назовем квазиизоморфизмом, если его конус является ацикличным комплексом. По-другому можно сказать, что $f$ - квазиизоморфизм, если отображение на когомологиях, которое он индуцирует, является изоморфизмом. Пусть Quis - это мультипликативная система, ассоциированная с $\mathscr{N}$, т.е. система, состояшая из всех квазиизоморфизмов. 
ОПРЕДЕЛЕНИЕ 1.2.3. Производная категория $\mathbf{D}(\mathscr{A})$ от абелевой категории $\mathscr{A}$ есть по определению локализация гомотопической категории $\mathbf{H}(\mathscr{A})$ по подкатегории всех ацикличных комплексов, т.е.

$$
\mathbf{D}(\mathscr{A}):=\mathbf{H}(\mathscr{A}) / \mathscr{N}=\mathbf{H}(\mathscr{A})\left[\mathrm{Quis}^{-1}\right] .
$$

Для $* \in\{+,-, b\}$ аналогично определим соответствующую производную категорию $\mathbf{D}^{*}(\mathscr{A})$ как локализацию $\mathbf{H}^{*}(\mathscr{A}) /\left(\mathbf{H}^{*}(\mathscr{A}) \cap \mathscr{N}\right)$.

ЛЕмма 1.2.4. Канонические функторы

$$
\mathbf{D}^{*}(\mathscr{A}) \longrightarrow \mathbf{D}(\mathscr{A}), \quad * \in\{+,-, b\}
$$

задают эквивалентности с полными подкатегориями в $\mathbf{D}(\mathscr{A})$, которые образованы комплексами ацикличными соответственно при $n \ll 0$, при $n \gg 0$, при $n \ll 0$ и $n \gg 0$. Подкатегория $\mathbf{H}^{+}(\mathscr{A})\left(\right.$ соответственно $\left.\mathbf{H}^{-}(\mathscr{A})\right)$ является кофинальной справа (соответственно слева) в $\mathbf{H}(\mathscr{A})$ относительно класса квазиизоморфизмов.

Предположим, что абелева категория $\mathscr{A}$ имеет достаточно много инъективных, т.е. любой объект допускает вложение в инъективньй. Обозначим через $\mathscr{I}$ полную подкатегорию в $\mathscr{A}$, состояшую из инъективных объектов. В этом случае можно показать, что сквозной функтор

$$
\mathbf{H}^{+}(\mathscr{I}) \longrightarrow \mathbf{H}^{+}(\mathscr{A}) \stackrel{Q}{\longrightarrow} \mathbf{D}^{+}(\mathscr{A})
$$

является эквивалентностью категорий (см. [17]). Аналогично, если абелева категория $\mathscr{A}$ имеет достаточно много проективных, то сквозной функтор

$$
\mathbf{H}^{-}(\mathscr{P}) \longrightarrow \mathbf{H}^{-}(\mathscr{A}) \stackrel{Q}{\longrightarrow} \mathbf{D}^{-}(\mathscr{A})
$$

где $\mathscr{P}$ в $\mathscr{A}$ - полная подкатегория проективных, является эквивалентностью.

Пусть $F: \mathscr{A} \longrightarrow \mathscr{B}$ - некоторьй аддитивньй функтор между абелевыми категориями (точность не требуется). Тогда он очевидным образом индуцирует точный функтор $\mathbf{H}(\mathscr{A}) \longrightarrow \mathbf{H}(\mathscr{B})$, которьй будем обозначать тем же символом $F$. Общая конструкция (правого) производного функтора, описанная в предыдущем разделе, дает нам функтор $\mathbf{R} F$, которьй определен на некоторой полной триангулированной подкатегории в $\mathbf{D}(\mathscr{A})$, со значениями в $\mathbf{D}(\mathscr{B})$. Те же самые слова можно сказать и про левьй производньй функтор. Определим $n$-й правьй (соответственно левый) производный функтор от $F$ как когомологии:

$$
\left.\mathbf{R}^{n} F X=H^{n}(\mathbf{R} F X) \quad \text { (соответственно } \mathbf{L}_{n} F X=H^{-n}(\mathbf{L} F X)\right), \quad n \in \mathbb{Z} .
$$

Обычно в приложениях правьй сопряженный функтор бывает хорошо определен на подкатегории $\mathbf{D}^{+}(\mathscr{A})$. Используя леммы 1.1.10 и 1.2.4, можно утверждать, что ограничение функтора $\mathbf{R} F$ на $\mathbf{D}^{+}(\mathscr{A})$ совпадает с функтором, производным от ограничения $F$ на $\mathbf{H}^{+}(\mathscr{A}) \subset \mathbf{H}(\mathscr{A})$.

Сейчас опишем условия, при которых правьй производньй функтор $\mathbf{R} F$ определен на всей категории $\mathbf{D}^{+}(\mathscr{A})$. Назовем полную аддитивную подкатегорию $\mathscr{R} \subset \mathscr{A} n p u-$ способленной справа к функтору $F$, если

a) функтор $F$ переводит ацикличные комплексы из $\mathbf{C}^{+}(\mathscr{R})$ в ацикличные;

b) любой объект из $\mathscr{A}$ вкладьвается в некоторьй объект из $\mathscr{R}$. 
Объекты категории $\mathscr{R}$ назьваются $F$-ацикличными справа. И если существует такая приспособленная справа к функтору $F$ категория $\mathscr{R}$, то часто говорят, что категория $\mathscr{A}$ имеет достаточно много $F$-ацикличных (справа) объектов.

Предположим, что сушествует приспособленная справа к функтору $F: \mathscr{A} \longrightarrow \mathscr{B}$ подкатегория $\mathscr{R} \subset \mathscr{A}$. Применяя лемму 1.1 .12$, нетрудно проверить, что любой ограниченньй справа комплекс объектов из $\mathscr{R}$ является $F$-расшепимым справа. Из условия b) легко вьвести, что для любого объекта $X \in \mathbf{H}^{+}(\mathscr{A})$ сушествует квазиизоморфизм $X \longrightarrow X^{\prime}$, где $X^{\prime} \in \mathbf{H}^{+}(\mathscr{R})$. Как следствие получаем, что канонический функтор

$$
\mathbf{H}^{+}(\mathscr{R})\left[\text { Quis }^{-1}\right] \longrightarrow \mathbf{D}^{+}(\mathscr{A})
$$

является эквивалентностью триангулированных категорий.

Лемма 1.2.5. Пусть существует приспособленная справа к функтору $F$ подкатегория $\mathscr{R} \subset \mathscr{A}$. Тогда функтор $\mathbf{R} F$ определен на $\mathbf{D}^{+}(\mathscr{A})$, для любого ограниченного слева комплекса $X$ имеется изоморфизм $\mathbf{R} F X \stackrel{\sim}{\longrightarrow} X^{\prime}$, әде $X \longrightarrow X^{\prime}-$ квазиизоморфизм с $X^{\prime} \in \mathbf{H}^{+}(\mathscr{A})$.

Если категория $\mathscr{A}$ имеет достаточно много инъективных, то полная подкатегория $\mathscr{I} \subset \mathscr{A}$, состоящая из всех инъективных, является приспособленной справа к любому аддитивному функтору. И в этом случае правый производный функтор $\mathbf{R} F X$ можно вычислять, применяя функтор $F$ к инъективной резольвенте $X^{\prime}$ комплекса $X$.

Двойственным образом можно определить понятие подкатегории, приспособленной слева к функтору $F$. И если такая подкатегория существует, то определен левый производньй функтор $\mathbf{L} F: \mathbf{D}^{-}(\mathscr{A}) \longrightarrow \mathbf{D}(\mathscr{B})$.

1.3. Производные категории пучков на схемах. Пусть $X$ - некоторая схема над полем $k$ со структурньм пучком $\mathscr{O}_{X}$. С каждой схемой можно связать несколько абелевых категорий пучков на ней. Обозначим через $\mathscr{O}_{X}$-Mod абелеву категорию всех пучков $\mathscr{O}_{X}$-модулей (в топологии Зариского). Категория $\mathscr{O}_{X}$-Mod имеет все пределы и все копределы и имеет множество порождающих. Прямые копределы являются точньми. По этой причине категория $\mathscr{O}_{X}$-Mod является абелевой категорией Гротендика и имеет достаточно много инъективных (см. [15], [42; Exp. IV]).

С этого момента ограничимся рассмотрением нётеровых схем (хотя многие факты, изложенные ниже вьполнены и в более общей ситуашии). Обозначим через $\mathrm{Qcoh}(X)$ полную абелеву подкатегорию в $\mathscr{O}_{X}$-Mod, состоящую из квазикогерентных пучков. Для нётеровой схемы $X$ всякий квазикогерентньй пучок является прямым копределом своих подпучков конечного типа (см. [16; EGA1, 9.4]). В этом случае категория $\mathrm{Q} \operatorname{coh}(X)$ имеет множество порождающих и является абелевой категорией Гротендика, поэтому имеет достаточно много инъективных.

Третья категория, которую можно сопоставить схеме $X,-$ это категория когерентных пучков $\operatorname{coh}(X)$. Категория $\operatorname{coh}(X)$ является полной абелевой подкатегорией в $\mathrm{Q} \operatorname{coh}(X)$.

Несмотря на то, что определение (квази)когерентных пучков локально, они на самом деле не зависят от топологии. К примеру, можно рассмотреть не только топологию Зариского, но и, скажем, этальную или плоскую топологии. В этом случае, в то время как понятие пучка $\mathscr{O}_{X}$-модулей зависит от выбора топологии, (квази)когерентные пучки не зависят (см. [36]). В частности, для аффиинной схемы категория $Q \operatorname{coh}(X)$ эквивалентна категории модулей над соответствуюшей алгеброй. 
В дальнейшем наше внимание будет сосредоточено на изучении категории когерентных пучков, а более точно, на производной категории когерентных пучков. Однако, так как категория $\operatorname{coh}(X)$ не имеет достаточно много инъективных, то при построении производных функторов нам будут полезны категории $Q \operatorname{coh}(X)$ и $\mathscr{O}_{X}$-Mod.

Для нётеровой схемы $X$ полное вложение абелевых категорий $\mathrm{Qcoh}(X) \hookrightarrow \mathscr{O}_{X}$-Mod переводит инъективные в инъективные. Отсюда несложной процедурой (см. [17; I.4.6], [43; приложение B]) можно вьвести, что триангулированная подкатегория $\mathbf{H}^{+}(\mathrm{Qcoh})$ является кофинальной справа в триангулированной категории $\mathbf{H}^{+}\left(\mathscr{O}_{X}\right.$-Mod). Поэтому, применяя лемму 1.1.6, получаем следующее утверждение.

ПРЕДЛОЖЕНИЕ 1.3.1 [17], [41; Ехр. II]. Если $X$ - нётерова схема, то канонический функтор

$$
\mathbf{D}^{+}(\mathrm{Q} \operatorname{coh}(X)) \longrightarrow \mathbf{D}^{+}\left(\mathscr{O}_{X}-\mathrm{Mod}\right)
$$

является вполне строгим и задает әквивалентность с полной подкатегорией

$$
\mathbf{D}^{+}\left(\mathscr{O}_{X}-\operatorname{Mod}\right)_{\mathrm{Qcoh}} \subset \mathbf{D}^{+}\left(\mathscr{O}_{X}-\operatorname{Mod}\right)
$$

состоящей из комплексов с квазикогерентными когомологиями.

При дополнительных ограничениях на схему можно доказать аналогичное утверждение и про неограниченные производные категории.

ПРЕДЛОЖЕНИЕ 1.3 .2 [41; Ехр. II]. Если X - нётерова схема конечной размерности, то канонический функтор

$$
\mathbf{D}(\mathrm{Q} \operatorname{coh}(X)) \longrightarrow \mathbf{D}\left(\mathscr{O}_{X}-\mathrm{Mod}\right)
$$

является вполне строгим и задает эквивалентность с полной подкатегорией

$$
\mathbf{D}\left(\mathscr{O}_{X}-\mathrm{Mod}\right)_{\mathrm{Qcoh}} \subset \mathbf{D}\left(\mathscr{O}_{X}-\mathrm{Mod}\right)
$$

состоящей из комплексов с квазикогерентными когомологиями.

Доказательство использует наличие функтора $Q: \mathscr{O}_{X}-\operatorname{Mod} \longrightarrow \mathrm{Q} \operatorname{coh}(X)$, coпряженного справа к функтору вложения, и тот факт, что для схемы конечной размерности этот функтор имеет конечную когомологическую размерность (см. [41; II.3.7]).

Теперь рассмотрим вложение абелевых категорий $\operatorname{coh}(X) \subset \mathrm{Q} \operatorname{coh}(X)$. Для канонического функтора между производными категориями также известны утверждения, аналогичные описанньм выше. Однако они касаются только ограниченных справа производных категорий.

ПРЕДЛОЖЕНИЕ 1.3 .3 [41; Ехр. II]. Для нётеровой схемы X канонический функmop

$$
\mathbf{D}^{-}(\operatorname{coh}(X)) \longrightarrow \mathbf{D}^{-}(\mathrm{Q} \operatorname{coh}(X))
$$

является вполне строгим и задает эквивалентность с полной подкатегорией

$$
\mathbf{D}^{-}(\mathrm{Q} \operatorname{coh}(X))_{\operatorname{coh}}
$$

Комбинируя это предложение с предложениями 1.3 .1 и 1.3.2, получаем следствие. 
СлЕДСТВИЕ 1.3 .4 [41; Ехр. II]. Пусть X - нётерова схема (соответственно нётерова конечной размерности). Тогда канонический функтор

$\mathbf{D}^{b}(\operatorname{coh}(X)) \longrightarrow \mathbf{D}^{b}\left(\mathscr{O}_{X}-\mathrm{Mod}\right) \quad\left(\right.$ соответственно $\left.\mathbf{D}^{-}(\operatorname{coh}(X)) \longrightarrow \mathbf{D}^{-}\left(\mathscr{O}_{X}-\mathrm{Mod}\right)\right)$

является вполне строгим и задает әквивалентность с полной подкатегорией

$$
\mathbf{D}^{b}\left(\mathscr{O}_{X}-\mathrm{Mod}\right)_{\mathrm{coh}} \quad\left(\text { соответственно } \mathbf{D}^{-}\left(\mathscr{O}_{X}-\mathrm{Mod}\right)_{\mathrm{coh}}\right)
$$

Теперь опишем основные производные функторы между производными категориями пучков на схемах. Пусть $f: X \longrightarrow Y$-морфизм нётеровых схем. Сушествует функтор обратного образа

$$
f^{*}: \mathscr{O}_{Y}-\operatorname{Mod} \longrightarrow \mathscr{O}_{X}-\operatorname{Mod},
$$

которьй точен справа. Так как категория $\mathscr{O}_{Y}$-Mod имеет достаточно много плоских

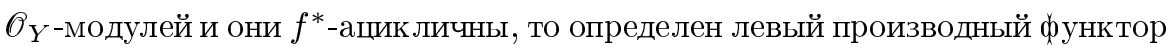

$$
\mathbf{L} f^{*}: \mathbf{D}^{-}\left(\mathscr{O}_{Y}-\mathrm{Mod}\right) \longrightarrow \mathbf{D}^{-}\left(\mathscr{O}_{X^{-}} \text {Mod }\right)
$$

Нетрудно показать, что функтор $\mathbf{L} f^{*}$ переводит категории $\mathbf{D}^{-}\left(\mathscr{O}_{Y}-\mathbf{M o d}\right)_{\mathrm{Qcoh}}$ и $\mathbf{D}^{-}\left(\mathscr{O}_{Y}-\mathrm{Mod}\right)_{\text {coh в категории }} \mathbf{D}^{-}\left(\mathscr{O}_{X}-\mathrm{Mod}\right)_{\mathrm{Qcoh}}$ и $\mathbf{D}^{-}\left(\mathscr{O}_{X}-\mathrm{Mod}\right)_{\text {coh }}$ соответственно. Таким образом, для нётеровых схем конечной размерности получается производньй функтор обратного образа на ограниченных справа производных категориях квазикогерентных и когерентных пучков.

Если функтор $f^{*}$ имеет конечную когомологическую размерность (в этом случае будем говорить, что $f$ имеет конечную Tor-размерность) можно продолжить производный функтор $\mathbf{L} f^{*}$ на неограниченные производные категории. Более того, если морфизм $f$ имеет конечную Tor-размерность, то производный функтор обратного образа переводит ограниченную производную категорию в ограниченную. И, в частности, имеем функтор

$$
\mathbf{L} f^{*}: \mathbf{D}^{b}\left(\mathscr{O}_{Y}-\operatorname{Mod}\right)_{\mathrm{coh}} \longrightarrow \mathbf{D}^{b}\left(\mathscr{O}_{X}-\operatorname{Mod}\right)_{\mathrm{coh}} .
$$

Пусть $\mathscr{E}, \mathscr{F} \in \mathbf{C}\left(\mathscr{O}_{X}\right.$-Mod $)$ - два комплекса $\mathscr{O}_{X}$-модулей. Определим тензорное произведение $\mathscr{E} \otimes \mathscr{F}$ как комплекс, ассоциированньй с двойным комплексом $\mathscr{E} p \otimes \mathscr{F}$, т.е.

$$
(\mathscr{E} \otimes \mathscr{F})^{n}=\sum_{p+q=n} \mathscr{E}^{p} \otimes \mathscr{F}^{q}
$$

с дифференциалом $d=d_{\mathscr{E}}+(-1)^{n} d \mathscr{F}$. Гомотопии между морфизмами комплексов переносятся на тензорное произведение, и получается функтор

$$
\mathscr{E} \otimes: \mathbf{H}\left(\mathscr{O}_{X}-\mathrm{Mod}\right) \longrightarrow \mathbf{H}\left(\mathscr{O}_{X}-\mathrm{Mod}\right)
$$

Предположим теперь, что $\mathscr{E} \in \mathbf{C}^{-}\left(\mathscr{O}_{X}-\mathrm{Mod}\right)$. Категория $\mathbf{H}^{-}\left(\mathscr{O}_{X}-\mathrm{Mod}\right)$ имеет достаточно много объектов, расшепимых слева для функтора $\mathscr{E} \otimes$. Таковьми являются 
ограниченные справа комплексы плоских $\mathscr{O}_{X}$-модулей. Поэтому существует левьй производньй функтор

$$
\stackrel{\mathscr{L}}{\otimes}: \mathbf{D}^{-}\left(\mathscr{O}_{X}-\mathrm{Mod}\right) \longrightarrow \mathbf{D}^{-}\left(\mathscr{O}_{X}-\text { Mod }\right)
$$

Если объекты $\mathscr{E}_{1}$ и $\mathscr{E}_{2}$ квазиизоморфны, то функторы $\mathscr{E}_{1}^{\stackrel{\mathrm{L}}{\otimes}}$ и $\mathscr{E}_{2} \stackrel{\text { L }}{\otimes}$ являются изоморфными. На самом деле, получается функтор от двух переменных

$$
\stackrel{\mathbf{L}}{\otimes}: \mathbf{D}^{-}\left(\mathscr{O}_{X}-\mathrm{Mod}\right) \times \mathbf{D}^{-}\left(\mathscr{O}_{X}-\operatorname{Mod}\right) \longrightarrow \mathbf{D}^{-}\left(\mathscr{O}_{X}-\mathrm{Mod}\right),
$$

которьй точен по обоим аргументам. Производньй функтор тензорного произведения очевидно симметричный и ассоциативный.

Предположим, что объект $\mathscr{E}$ имеет конечную Tor-размерность, то есть квазиизоморфен ограниченному комплексу плоских $\mathscr{O}_{X}$-модулей, тогда функтор $\mathscr{E} \otimes$ можно, с одной стороны, продолжить на неограниченную производную категорию, а с другой, путем ограничения получить функтор из ограниченной производной категории в себя. Получаем функторы

$$
\mathscr{E} \otimes: \mathbf{D}\left(\mathscr{O}_{X}-\text { Mod }\right) \longrightarrow \mathbf{D}\left(\mathscr{O}_{X}-\operatorname{Mod}\right), \quad \mathscr{E} \otimes: \mathbf{D}^{b}\left(\mathscr{O}_{X}-\operatorname{Mod}\right) \longrightarrow \mathbf{D}^{b}\left(\mathscr{O}_{X}-\operatorname{Mod}\right)
$$

Отметим, что если объект $\mathscr{E}$ принадлежит категории $\mathbf{D}^{-}\left(\mathscr{O}_{X}-\mathrm{Mod}\right)_{\mathrm{coh}}(\mathrm{coot-}$ ветственно $\left.\mathbf{D}^{-}\left(\mathscr{O}_{X}-\mathrm{Mod}\right)_{\mathrm{Qcoh}}\right)$, то функтор $\mathscr{E} \otimes$ 离 переводит объекты с (квази)когерентными когомологиями в объекты с (квази)когерентньми когомологиями. Пусть $f: X \longrightarrow Y$-некоторый морфизм нётеровых схем. Функтор прямого образа

$$
f_{*}: \mathscr{O}_{X}-\operatorname{Mod} \longrightarrow \mathscr{O}_{Y}-\operatorname{Mod}
$$

является точньм слева. Так как категория $\mathscr{O}_{X}$-модулей имеет достаточно много инъективных, то существует правьй производньй функтор

$$
\mathbf{R} f_{*}: \mathbf{D}^{+}\left(\mathscr{O}_{X}-\mathrm{Mod}\right) \longrightarrow \mathbf{D}^{+}\left(\mathscr{O}_{X}-\operatorname{Mod}\right) .
$$

Более того, в этом случае функтор $\mathbf{R} f_{*}$ переводит подкатегорию $\mathbf{D}^{+}\left(\mathscr{O}_{X}-\mathrm{Mod}\right)_{\mathrm{Qcoh}}$ в подкатегорию $\mathbf{D}^{+}\left(\mathscr{O}_{Y}-\mathrm{Mod}\right)_{\mathrm{Qcoh}}$.

Если дополнительно функтор $f_{*}$ имеет конечную когомологическую размерность, то функтор $\mathbf{R} f_{*}$ можно продолжить на категорию неограниченных комплексов. Это вьполняется, например, в случае, когда $X$ - нётерова схема конечной размерности. С другой стороны, в этом случае, т.е. когда $f_{*}$ имеет конечную когомологическую размерность, определен правый производный функтор между ограниченными производньми категориями

$$
\mathbf{R} f_{*}: \mathbf{D}^{b}\left(\mathscr{O}_{X}-\mathrm{Mod}\right) \longrightarrow \mathbf{D}^{b}\left(\mathscr{O}_{X}-\operatorname{Mod}\right)
$$

Для того чтобы правый производньй функтор был определен между производными категориями когерентных пучков, необходимы дополнительные ограничения на морфизм. 
ПРЕДЛОЖЕНИЕ 1.3.5 [16; III, 3.2.1], [17]. Предположим, что $f: X \longrightarrow Y$ является собственным морфизмом нётеровых схем. Тогда функтор $\mathbf{R} f_{*}$ переводит подкатегорию $\mathbf{D}^{+}\left(\mathscr{O}_{X}-\mathrm{Mod}\right)_{\mathrm{coh}}$ в подкатегорию $\mathbf{D}^{+}\left(\mathscr{O}_{Y}-\mathrm{Mod}\right)_{\mathrm{coh}}$. Eсли, кроме того, $X$ имеет конечную размерность, то аналогичное утверэсдение выполняется для ограниченной и неограниченной производных категорий.

Пусть $\mathscr{E}, \mathscr{F} \in \mathbf{C}\left(\mathscr{O}_{X}\right.$-Mod $)$ - два комплекса $\mathscr{O}_{X}$-модулей. Определим комплекс $\underline{\mathscr{H} o m} \cdot(\mathscr{E}, \mathscr{F})$ по правилу:

$$
\underline{\mathscr{H} o m}^{n}(\mathscr{E}, \mathscr{F})=\prod_{p} \underline{\mathscr{H} o m}\left(\mathscr{E} p, \mathscr{F}^{p+n}\right)
$$

с дифференциалом $d=d_{\mathscr{E}}+(-1)^{n+1} d_{\mathscr{F}}$. Гомотопии между морфизмами комплексов переносятся на локальньй $\underline{\mathscr{H} о \mathrm{~m}}$, и получается бифунктор

$$
\underline{\mathscr{H} o m}: \mathbf{H}\left(\mathscr{O}_{X}-\mathrm{Mod}\right)^{o p} \times \mathbf{H}\left(\mathscr{O}_{X}-\mathrm{Mod}\right) \longrightarrow \mathbf{H}\left(\mathscr{O}_{X}-\mathrm{Mod}\right)
$$

Так как любой ограниченный слева комплекс имеет инъективную резольвенту, то получаем производный бифунктор

$$
\mathbf{R} \underline{\mathscr{H} o m}: \mathbf{D}\left(\mathscr{O}_{X}-\mathrm{Mod}\right)^{o p} \times \mathbf{D}^{+}\left(\mathscr{O}_{X}-\mathrm{Mod}\right) \longrightarrow \mathbf{D}\left(\mathscr{O}_{X}-\mathrm{Mod}\right) .
$$

В данной ситуации определим локальньй гипер-Ext

$$
\underline{\mathscr{E} x t}{ }^{i}(\mathscr{E}, \mathscr{F}):=H^{i}(\mathbf{R} \underline{\mathscr{H} o m}(\mathscr{E}, \mathscr{F})) .
$$

Для нётеровой схемы $X$ если $\mathscr{E}$ и $\mathscr{F}$ являются (квази)когерентными $\mathscr{O}_{X}$-модулями, тогда для всех $i \geqslant 0$ пучки $\underline{\mathscr{E} x t} t^{i}(\mathscr{E}, \mathscr{F})$ также являются (квази)когерентными.

Если теперь $\mathscr{E} \in \mathbf{D}^{-}\left(\mathscr{O}_{X}-\mathrm{Mod}\right)_{\mathrm{coh}}$, a $\mathscr{F} \in \mathbf{D}^{+}\left(\mathscr{O}_{X}-\mathrm{Mod}\right)_{\mathrm{coh}}$, тогда $\mathbf{R} \underline{\mathscr{H} о \mathrm{~m}}(\mathscr{E}, \mathscr{F})$ принадлежит $\mathbf{D}\left(\mathscr{O}_{X}-\mathrm{Mod}\right)_{\mathrm{coh}}$.

Опишем основные свойства и соотношения между производньми функторами, введенньми в этом разделе. Рассмотрим два морфизма $f: X \longrightarrow Y$ и $g: Y \longrightarrow Z$. В данной ситуации имеем два функтора $\mathbf{L}(g f)^{*}$ и $\mathbf{L} f^{*} \mathbf{L} g^{*}$ из категории $\mathbf{D}^{-}\left(\mathscr{O}_{Z}-\mathrm{Mod}\right)$ в $\mathbf{D}^{-}\left(\mathscr{O}_{X}\right.$-Mod). Тогда естественное преобразование

$$
\mathbf{L}(g f)^{*} \stackrel{\sim}{\longrightarrow} \mathbf{L} f^{*} \mathbf{L} g^{*}
$$

является изоморфизмом. Доказательство этого факта следует из того, что функтор $g^{*}$ переводит плоские $\mathscr{O}_{Z}$-модули в плоские $\mathscr{O}_{Y}$-модули (см., например, [17]).

Аналогично, имеем изоморфизм

$$
\mathbf{R}(g f)_{*} \longrightarrow \mathbf{R} g_{*} \mathbf{R} f_{*}
$$

функторов из $\mathbf{D}^{+}\left(\mathscr{O}_{X}-\mathrm{Mod}\right)$ в $\mathbf{D}^{+}\left(\mathscr{O}_{Z}-\mathrm{Mod}\right)$. Данное утверждение следует из того факта, что $f_{*}$ переводит инъективные пучки в вялые пучки на $Y$, которые, в свою очередь, являются $g_{*}$-ацикличными (см. [17]).

Другие соотношения, которые будут довольно часто использоваться, назьваются формулой проекции и плоской заменой базы. 
ПРЕДЛОЖЕНИЕ 1.3.6 [17; II.5.6]. Пусть $f: X \longrightarrow Y-м$ морфизм нётеровьх схем конечной размерности. Тогда для любых облектов $\mathscr{E} \in \mathbf{D}^{-}\left(\mathscr{O}_{Z}\right.$-Mod $)$ и $\mathscr{F} \in$ $\mathbf{D}^{-}\left(\mathscr{O}_{X}-\mathrm{Mod}\right)_{\mathrm{Qcoh}}$ существует естественный изоморфизм функторов

$$
\mathbf{R} f_{*} \mathscr{E} \otimes \mathscr{L} \mathscr{F} \stackrel{\sim}{\longrightarrow} \mathbf{R} f_{*}\left(\mathscr{E} \otimes \mathbf{L} \otimes \mathbf{L} f^{*} \mathscr{F}\right)
$$

ПРЕДЛОЖЕНИЕ 1.3 .7 [17; II.5.12]. Пусть $f: X \longrightarrow Y-$ морфизм конечного типа межсду нётеровыми схемами конечной размерности и $g: Y^{\prime} \longrightarrow Y-$ плоский морфизм. Рассмотрим декартов квадрат:

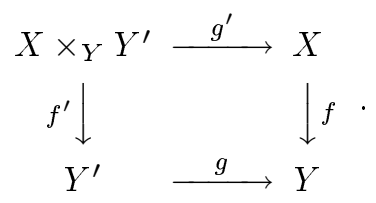

В данной ситуации существует естественный изоморфизм функторов

$$
\mathbf{L} g^{*} \mathbf{R} f_{*} \mathscr{E} \stackrel{\sim}{\longrightarrow} f_{*}^{\prime} \mathbf{L} g^{\prime} \mathscr{E}^{\prime}
$$

для любого $\mathscr{E} \in \mathbf{D}\left(\mathscr{O}_{X}-\mathrm{Mod}\right)_{\mathrm{Qcoh}}$.

Сформулируем еше одно соотношение, которое нам понадобится.

ПРЕДЛОЖЕНИЕ 1.3 .8 [17; II.5.16]. Пусть $\mathscr{E}$ - ограниченный комплекс локально свободных пучков конечного ранга на нётеровой схеме $X$. Тогда существуют естественные изоморфизмы функторов

$$
\mathbf{R} \underline{\mathscr{H} o m}(\mathscr{F}, \mathscr{G}) \stackrel{\mathrm{L}}{\otimes} \mathscr{E} \stackrel{\sim}{\longrightarrow} \underline{\mathbf{H} o m}(\mathscr{F}, \mathscr{G} \stackrel{\mathrm{L}}{\otimes} \mathscr{E}) \stackrel{\sim}{\longrightarrow} \mathbf{R} \underline{\mathscr{H} o m}(\mathscr{F} \stackrel{\mathbf{L}}{\otimes} \mathscr{E} \vee, \mathscr{G})
$$

для всех $\mathscr{F} \in \mathbf{D}^{-}\left(\mathscr{O}_{X}-\mathrm{Mod}\right)$ u $\mathscr{G} \in \mathbf{D}^{+}\left(\mathscr{O}_{X}-\mathrm{Mod}\right)$, дде $\mathscr{E} \vee:=\mathbf{R} \underline{\mathscr{H} o m}\left(\mathscr{E}, \mathscr{O}_{X}\right)$.

\section{Глава 2. Категории когерентных пучков и функторы между ними}

2.1. Основные свойства категории когерентных пучков. Начиная с этого раздела, будем рассматривать только ограниченные производные категории когерентных пучков на гладких полных алгебраических многообразиях. Для краткости вместо $\mathbf{D}^{b}(\operatorname{coh}(X))$ далее везде будем писать просто $\mathbf{D}^{b}(X)$. Кроме того, значок производного функтора будет опускаться в тех случаях, когда функтор точен, например, для обратного образа при плоском морфизме или для тензорного умножения на локально свободньй пучок.

Для гладкого полного многообразия $X$ размерности $n$ ограниченная производная категория когерентных пучков обладает функтором Серра (см. определение 1.1.3), и этот функтор совпадает с функтором $(\cdot) \otimes \omega_{X}[n]$, где $\omega_{X}-$ канонический пучок (см. [6]). Таким образом, имеется изоморфизм

$$
\operatorname{Hom}(\mathscr{E}, \mathscr{F})=\operatorname{Hom}\left(\mathscr{F}, \mathscr{E} \otimes \omega_{X}[n]\right)^{*}
$$

для любой пары объектов $\mathscr{E}, \mathscr{F} \in \mathbf{D}^{b}(X)$. 
Как было показано в предыдущем разделе, любой морфизм $f: X \rightarrow Y$ между гладкими полньми алгебраическими многообразиями индуцирует два точных функтора: функтор прямого образа $\mathbf{R} f_{*}: \mathbf{D}^{b}(X) \longrightarrow \mathbf{D}^{b}(Y)$ и функтор обратного образа $\mathbf{L} f^{*}: \mathbf{D}^{b}(Y) \longrightarrow \mathbf{D}^{b}(X)$, и эти функторы сопряжены. Кроме того, каждьй объект $\mathscr{E} \in \mathbf{D}^{b}(X)$ задает точный функтор тензорного умножения $\stackrel{\mathbf{L}}{\otimes} \mathscr{E}: \mathbf{D}^{b}(X) \longrightarrow \mathbf{D}^{b}(X)$.

Используя эти стандартные производные функторы, можно ввести новый большой класс точных функторов между производньми категориями $\mathbf{D}^{b}(X)$ и $\mathbf{D}^{b}(Y)$.

Пусть $X$ и $Y$ - два гладких полных многообразия над полем $k$ размерности $n$ и $m$ соответственно. Рассмотрим декартово произведение $X \times Y$ и обозначим через $p$ и $q$ проекции $X \times Y$ на $X$ и, соответственно, на $Y$ :

$$
X \stackrel{p}{\stackrel{1}{\leftrightarrows}} X \times Y \stackrel{q}{\longrightarrow} Y .
$$

Каждый объект $\mathscr{E} \in \mathbf{D}^{b}(X \times Y)$ задает точный функтор $\Phi_{\mathscr{E}}$ из производной категории $\mathbf{D}^{b}(X)$ в производную категорию $\mathbf{D}^{b}(Y)$, которьй определяется следующей формулой:

$$
\Phi_{\mathscr{E}}(\cdot):=\mathbf{R}^{\cdot} q_{*}\left(\mathscr{E} \stackrel{\mathbf{L}}{\otimes} p^{*}(\cdot)\right) .
$$

Кроме того, с тем же самым объектом $\mathscr{E} \in \mathbf{D}^{b}(X \times Y)$ можно связать еше и другой функтор $\Psi_{\mathscr{E}}$ из производной категории $\mathbf{D}^{b}(Y)$ в производную категорию $\mathbf{D}^{b}(X)$, определенньй по правилу, аналогичному (7):

$$
\Psi_{\mathscr{E}}(\cdot):=\mathbf{R} p_{*}\left(\mathscr{E} \stackrel{\mathbf{L}}{\otimes} q^{*}(\cdot)\right)
$$

Легко проверить, что функтор $\Phi_{\mathscr{E}}$ имеет как левьй, так и правьй сопряженные функторы.

Лемма 2.1.1. Функтор $\Phi_{\mathscr{E}}$ имеет сопряженные слева и справа функторы $\Phi_{\mathscr{E}}^{*}$ и $\Phi_{\mathscr{E}}^{!}$соответственно, которые задаются по формуле:

$$
\begin{aligned}
& \Phi_{\mathscr{E}}^{*} \cong \Psi_{\mathscr{E} \vee} \otimes q^{*} \omega_{Y}[m] \\
& \Phi_{\mathscr{E}}^{!} \cong \Psi_{\mathscr{E} \vee} \vee p^{*} \omega_{X}[n]
\end{aligned}
$$

Здесь $\omega_{X}$ и $\omega_{Y}$ канонические пучки на $X$ и $Y$, и $\mathscr{E}^{\vee}-$ удобное обозначение для $\mathbf{R} \underline{\mathscr{H} O m}\left(\mathscr{E}, \mathscr{O}_{X \times Y}\right)$.

ДокАЗАТЕЛЬство. Приведем доказательство для сопряженного слева функтора. Оно получается из следуюшей последовательности изоморфизмов:

$$
\begin{aligned}
\operatorname{Hom}\left(A, \mathbf{R} q_{*}\left(\mathscr{E} \otimes p^{*} B\right)\right) & \cong \operatorname{Hom}\left(q^{*} A, \mathscr{E} \stackrel{\mathbf{L}}{\otimes} p^{*} B\right) \\
& \cong \operatorname{Hom}\left(p^{*} B, \mathscr{E}^{\vee} \stackrel{\mathbf{L}}{\otimes} q^{*} A \otimes \omega_{X \times Y}[n+m]\right)^{*} \\
& \cong \operatorname{Hom}\left(B, \mathbf{R} p_{*}\left(\mathscr{E} \vee \stackrel{\mathbf{L}}{\otimes} q^{*}\left(A \otimes \omega_{Y}[m]\right)\right) \otimes \omega_{X}[n]\right)^{*} \\
& \cong \operatorname{Hom}\left(\mathbf{R} p_{*}\left(\mathscr{E}^{\vee} \stackrel{\mathbf{L}}{\otimes} q^{*}\left(A \otimes \omega_{Y}[m]\right)\right), B\right) .
\end{aligned}
$$

Здесь используется сопряженность функторов прямого и обратного образа, два раза используется двойственность Серра (6), а также формула (5). 
Стоит отметить, что, конечно, с любой диаграммой вида

$$
X \stackrel{p}{\longleftarrow} Z \stackrel{q}{\longrightarrow} Y
$$

и любым объектом $\mathscr{E} \in \mathbf{D}^{b}(Z)$ можно связать функтор из производной категории когерентных пучков на $X$ в производную категорию когерентных пучков на $Y$, задаюшийся по формуле аналогичной (7). Но каждьй такой функтор изоморфен функтору вида (7) с объектом $\mathbf{R}(p, q) * \mathscr{E}$ на $X \times Y$, где $(p, q)$ - канонический морфизм из $Z$ в прямое произведение $X \times Y$.

Пусть теперь $X, Y, Z$ - три гладких полных многообразия и $\mathscr{E}, \mathscr{F}, \mathscr{G}$ - объекты производных категорий $\mathbf{D}^{b}(X \times Y), \mathbf{D}^{b}(Y \times Z)$ и $\mathbf{D}^{b}(X \times Z)$ соответственно. Рассмотрим следующую диаграмму проекций:

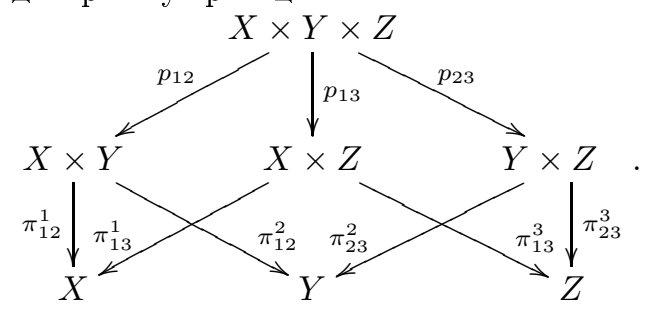

Объекты $\mathscr{E}, \mathscr{F}, \mathscr{G}$ задают три функтора:

$$
\Phi_{\mathscr{E}}: \mathbf{D}^{b}(X) \longrightarrow \mathbf{D}^{b}(Y), \quad \Phi_{\mathscr{F}}: \mathbf{D}^{b}(Y) \longrightarrow \mathbf{D}^{b}(Z), \quad \Phi_{\mathscr{G}}: \mathbf{D}^{b}(X) \longrightarrow \mathbf{D}^{b}(Z),
$$

определенные формулой (7), т.е.

$\Phi_{\mathscr{E}}:=\mathbf{R} \pi_{12 *}^{2}\left(\stackrel{\mathscr{E}}{\otimes} \otimes \pi_{12}^{1}{ }^{*}(\cdot)\right), \quad \Phi_{\mathscr{F}}:=\mathbf{R} \pi_{23 *}^{3}\left(\stackrel{\mathscr{F}}{\mathbf{L}} \pi_{23}^{2}{ }^{*}(\cdot)\right), \quad \Phi_{\mathscr{G}}:=\mathbf{R} \pi_{13 *}^{3}\left(\stackrel{\mathscr{G}}{\otimes} \otimes \pi_{13}^{1}{ }^{*}(\cdot)\right)$.

Рассмотрим объект $p_{12}^{*} \mathscr{E} \stackrel{\mathbf{L}}{\otimes} p_{23}^{*} \mathscr{F} \in \mathbf{D}^{b}(X \times Y \times Z)$, которьй далее всегда будем обозначать $\mathscr{E} \underset{Y}{\bigotimes} \mathscr{F}$. Следуюшее утверждение дает правило для композиции тех точных функторов между производными категориями, которые представлены объектами на произведении.

ПРЕДЛОЖЕНИЕ 2.1.2. Композииия функторов $\Phi_{\mathscr{F}} \circ \Phi_{\mathscr{E}}$ изоморфна функтору $\Phi_{\mathscr{G}}$, представленному обгектом

$$
\mathscr{G}=\mathbf{R} p_{13 *}(\underset{Y}{\mathbb{Q}} \underset{\mathcal{F}}{\mathscr{T}})
$$

Доказательство - это прямая проверка.

Таким образом, каждому гладкому полному алгебраическому многообразию сопоставляется производная категория когерентных пучков на нем, а с каждым объектом $\mathscr{E} \in \mathbf{D}^{b}(X \times Y)$ на произведении двух таких многообразий ассоциируется точный функтор $\Phi_{\mathscr{E}}$ из триангулированной категории $\mathbf{D}^{b}(X)$ в триангулированную категорию $\mathbf{D}^{b}(Y)$ с законом композиции, описанным вьше.

Следующие вопросы являются фундаментальными для понимания данного сопоставления:

1) Когда производные категории когерентных пучков двух разных гладких полных алгебраических многообразий эквивалентны как триангулированные категории? 
2) Каковагруппа точных автоэквивалентностей производной категории когерентных пучков для данного фиксированного многообразия $X$ ? (Под групой автоэквивалентностей подразумевается группа образованная классами изоморфизмов точных автоэквивалентностей.)

3) Всякий ли точньй функтор между производными категориями когерентных пучков представляется объектом на произведении, то есть имеет вид (7)?

Некоторые результаты в этом направлении уже известны. $\mathrm{K}$ примеру, можно дать исчерпывающий ответ на первые два вопроса в случае, когда канонический или антиканонический пучок многообразия обилен.

Теорема 2.1.3 [8]. Пусть $X$ - гладкое проективное многообразие, канонический (или антиканонический) пучок которого обилен. Предположсим, что категория $\mathbf{D}^{b}(X)$ эквивалентна как триангулированная категория производной категории $\mathbf{D}^{b}\left(X^{\prime}\right)$ для некоторого гладкого алгебраического многообразия $X^{\prime}$. Тогда многообразие $X^{\prime}$ изоморфно $X$.

Доказательство этой теоремы, которое приведено в [8], является конструктивным и дает метод восстановления многообразия по производной категории когерентных пучков. Более того, в условии теоремы можно предполагать, что производные категории эквивалентны не как триангулированные категории, а только как градуированные категории (см. [8]).

В этой ситуации можно также описать и группу точных автоэквивалентностей.

ТеОРема 2.1.4 [8]. Пусть $X-$ гладкое проективное многообразие, канонический (или антиканонический) пучок которого обилен. Тогда группа классов изоморфизмов точных автоэквивалентностей категории $\mathbf{D}^{b}(X)$ порожсдена автоморфизмами многообразия, подкрутками на линейные расслоения и сдвигами в производной категории.

Для любого многообразия $X$ группа точных автоэквивалентностей Auteq $\mathbf{D}^{b}(X)$ всегда содержит подгруппу $G(X)$, которая есть полупрямое произведение нормальной подгрупшы $G_{1}=\operatorname{Pic}(X) \oplus \mathbb{Z}$ и подгрупшы $G_{2}=\operatorname{Aut} X$, действуюшей естественным образом на $G_{1}$. При этом включении $G(X) \subset \operatorname{Auteq} \mathbf{D}^{b}(X)$ образующая $\mathbb{Z}$ переходит в функтор сдвига [1], линейное расслоение $\mathscr{L} \in \operatorname{Pic}(X)$ - в функтор $\otimes \mathscr{L}$, а автоморфизм $f: X \rightarrow X$ индуцирует автоэквивалентность $\mathbf{R} f_{*}$. В статье [8] доказано, что при условии, описанном в теореме 2.1.4, группа точных автоэквивалентностей Auteq $\mathbf{D}^{b}(X)$ совпадает с $G(X)$, т.е. в этом случае:

$$
\text { Auteq } \mathbf{D}^{b}(X) \cong \operatorname{Aut} X \ltimes(\operatorname{Pic}(X) \oplus \mathbb{Z}) \text {. }
$$

Чтобы исследовать вопрос, когда два многообразия имеют эквивалентные производные категории когерентных пучков, и описывать их группы автоэквивалентностей, желательно иметь явные формулы для всех точных функторов. Существует гипотеза, что все они представляются объектами на произведении, т.е. имеют вид (7). В следуюшей главе дается доказательство данной гипотезы для вполне строгих функторов и, в частности, для эквивалентностей. Собственно говоря, вся следующая глава будет посвяшена доказательству данного результата. Таким образом, это даст нам возможность рассматривать только функторы вида (7) при исследовании вопроса об 
эквивалентностях производных категорий когерентных пучков на гладких проективных многообразиях. Другая задача, которая встает в связи с решением этих проблем, - это необходимость иметь критерии для определения по функтору, является он эквивалентностью или нет. Чтобы проверить, что функтор $F$ является эквивалентностью, достаточно показать, что он и его правьй (или левьй) сопряженньй вполне строгие функторы (см. определение 1.1.7).

Сушествует некоторьй метод для определения того, когда функтор $\Phi_{\mathscr{E}}: \mathbf{D}^{b}(X) \longrightarrow$ $\mathbf{D}^{b}(Y)$ является вполне строгим.

ТеОРема 2.1.5 [7]. Пусть $M$ и $X$ - гладкие проективные многообразия над алгебраически замкнутым полем характеристики 0, и пусть $\mathscr{E} \in \mathbf{D}^{b}(M \times X)$. Тогда функтор $\Phi_{\mathscr{E}}$ является вполне строгим, если и только если выполняются следующие условия ортогональности:

1) $\operatorname{Hom}_{X}^{i}\left(\Phi_{\mathscr{E}}\left(\mathscr{O}_{t_{1}}\right), \Phi_{\mathscr{E}}\left(\mathscr{O}_{t_{2}}\right)\right)=0$ для всех $i$ u $t_{1} \neq t_{2}$

2) $\operatorname{Hom}_{X}^{0}\left(\Phi_{\mathscr{E}}\left(\mathscr{O}_{t}\right), \Phi_{\mathscr{E}}\left(\mathscr{O}_{t}\right)\right)=k$, $\operatorname{Hom}_{X}^{i}\left(\Phi_{\mathscr{E}}\left(\mathscr{O}_{t}\right), \Phi_{\mathscr{E}}\left(\mathscr{O}_{t}\right)\right)=0$ для $i \notin[0, \operatorname{dim} M]$.

Здесь $t, t_{1}, t_{2}$ - точки многообразия $M$ и $\mathscr{O}_{t_{i}}$ - соответствующие им пучки небоскребов.

В общем случае проверить вьполнение условий данной теоремы довольно трудно, однако в тех случаях, когда объект на произведении является векторным расслоением данньй критерий работает довольно успешно. Рассмотрим четыре гладких полных алгебраических многообразий $X_{1}, X_{2}, Y_{1}, Y_{2}$. Возьмем два объекта $\mathscr{E}_{1}$ и $\mathscr{E}_{2}$, принадлежащие категориям $\mathbf{D}^{b}\left(X_{1} \times Y_{1}\right)$ и $\mathbf{D}^{b}\left(X_{2} \times Y_{2}\right)$ соответственно, и рассмотрим объeKT

$$
\mathscr{E}_{1} \otimes \mathscr{E}_{2} \in \mathbf{D}^{b}\left(\left(X_{1} \times X_{2}\right) \times\left(Y_{1} \times Y_{2}\right)\right)
$$

которьй по определению есть $p_{13}^{*}\left(\mathscr{E}_{1}\right) \stackrel{\mathrm{L}}{\otimes} p_{24}^{*}\left(\mathscr{E}_{2}\right)$. Как и раньше (см. $\left.(7)\right)$, объекты $\mathscr{E}_{1}$, $\mathscr{E}_{2}, \mathscr{E}_{1} \bigotimes \mathscr{E}_{2}$ задают функторы:

$$
\begin{aligned}
\Phi_{\mathscr{E}_{1}} & : \mathbf{D}^{b}\left(X_{1}\right) \longrightarrow \mathbf{D}^{b}\left(Y_{1}\right), \\
\Phi_{\mathscr{E}_{2}} & : \mathbf{D}^{b}\left(X_{2}\right) \longrightarrow \mathbf{D}^{b}\left(Y_{2}\right), \\
\Phi_{\mathscr{E}_{1}} \otimes \mathscr{E}_{2} & : \mathbf{D}^{b}\left(X_{1} \times X_{2}\right) \longrightarrow \mathbf{D}^{b}\left(Y_{1} \times Y_{2}\right) .
\end{aligned}
$$

Рассмотрим некоторый объект $\mathscr{G} \in \mathbf{D}^{b}\left(X_{1} \times X_{2}\right)$ и обозначим через $\mathscr{H}$ объект $\Phi_{\mathscr{E}_{1} \otimes \mathscr{E}_{2}}(\mathscr{G}) \in \mathbf{D}^{b}\left(Y_{1} \times Y_{2}\right)$. С каждым из этих двух объектов можно связать по правилу (7) функторы

$$
\Phi_{\mathscr{G}}: \mathbf{D}^{b}\left(X_{1}\right) \longrightarrow \mathbf{D}^{b}\left(X_{2}\right), \quad \Phi_{\mathscr{H}}: \mathbf{D}^{b}\left(Y_{1}\right) \longrightarrow \mathbf{D}^{b}\left(Y_{2}\right)
$$

ПРЕДЛОЖЕНИЕ 2.1.6. В обозначениях, введенных выше, существует изоморфизм функторов $\Phi_{\mathscr{H}} \cong \Phi_{\mathscr{E}_{2}} \circ \Phi_{\mathscr{G}} \circ \Psi_{\mathscr{E}_{1}}$.

Доказательство немедленно следует из предложения 2.1.2.

Если теперь $Z_{1}, Z_{2}$ - еще два гладких полных многообразия и $\mathscr{F}_{1}, \mathscr{F}_{2}$ - объекты, соответственно, категорий $\mathbf{D}^{b}\left(Y_{1} \times Z_{1}\right)$ и $\mathbf{D}^{b}\left(Y_{2} \times Z_{2}\right)$, то имеются также функторы 
$\Phi_{\mathscr{F}_{1}}, \Phi_{\mathscr{F}_{2}}, \Phi_{\mathscr{F}_{1} \otimes \mathscr{F}_{2}}$. По правилу $(9)$ можно найти объекты $\mathscr{G}_{1}$ и $\mathscr{G}_{2}$, принадлежашие категориям $\mathbf{D}^{b}\left(X_{1} \times Z_{1}\right)$ и $\mathbf{D}^{b}\left(X_{2} \times Z_{2}\right)$ такие, что:

$$
\Phi_{\mathcal{G}_{1}} \cong \Phi_{\mathscr{F}_{1}} \circ \Phi_{\mathscr{E}_{1}}, \quad \Phi_{\mathscr{G}_{2}} \cong \Phi_{\mathscr{F}_{2}} \circ \Phi_{\mathscr{E}_{2}}
$$

Прямая проверка показывает, что сушествует естественное соотношение

$$
\Phi_{\mathscr{F}_{1} \otimes \mathscr{F}_{2}} \circ \Phi_{\mathscr{E}_{1} \otimes \mathscr{E}_{2}} \cong \Phi_{\mathscr{G}_{1} \otimes \mathscr{G}_{2}} .
$$

Используя его, легко доказать следующее утверждение.

ПРЕДЛОЖЕНИЕ 2.1.7. В условиях, описанных выше, предположим, что функторы $\Phi_{\mathscr{E}_{1}} u \Phi_{\mathscr{E}_{2}}$ являются вполне строгими (әквивалентностями). Тогда функmop

$$
\Phi_{\mathscr{E}_{1} \otimes \mathscr{E}_{2}}: \mathbf{D}^{b}\left(X_{1} \times X_{2}\right) \longrightarrow \mathbf{D}^{b}\left(Y_{1} \times Y_{2}\right)
$$

также является вполне строгим (әквивалентностью).

ДокАЗАТЕльство. Если функтор $F$ имеет сопряженньй, скажем, слева $F^{*}$, то он вполне строгий тогда и только тогда, когда композиция $F^{*} F$ изоморфна тождественному функтору. $\Phi$ ункторы $\Phi_{\mathscr{E}_{i}}$ имеют левые сопряженные $\Phi_{\mathscr{E}_{i}}^{*}$, определенные по формуле (8). Из того, что они вполне строгие, следует, что композиции $\Phi_{\mathscr{E}_{i}}^{*} \circ \Phi_{\mathscr{E}_{i}}$ изоморфны тождественным функторам, которые представляются структурньми пучками диагоналей $\Delta_{i} \in X_{i} \times X_{i}$. Легко проверить, что пучок $\mathscr{O}_{\Delta_{1}} \otimes \mathscr{O}_{\Delta_{2}}$ изоморфен структурному пучку диагонали $\mathscr{O}_{\Delta}$, где $\Delta$ - диагональ в $\left(X_{1} \times X_{2}\right) \times\left(X_{1} \times X_{2}\right)$. Используя формулу (10), получаем, что композиция $\Phi_{\mathscr{E}_{1} \otimes \mathscr{E}_{2}}^{*} \circ \Phi_{\mathscr{E}_{1} \otimes \mathscr{E}_{2}}$ представляется структурньм пучком диагонали $\Delta$ и, значит, изоморфна тождественному функтору. Таким образом, $\Phi_{\mathscr{E}_{1} \otimes \mathscr{E}_{2}}$ вполне строгий. Утверждение про эквивалентность доказывается аналогично.

Предположим сейчас, что функтор $\Phi_{\mathscr{E}}: \mathbf{D}^{b}(X) \longrightarrow \mathbf{D}^{b}(Y)$ является эквивалентностью и объект $\mathscr{F} \in \mathbf{D}^{b}(X \times Y)$ таков, что $\Psi_{\mathscr{F}} \cong \Phi_{\mathscr{E}}^{-1}$. По формулам (8) имеются изоморфизмы

$$
\mathscr{F} \cong \mathscr{E}^{\vee} \otimes p^{*} \omega_{X}[n] \cong \mathscr{E}^{\vee} \otimes q^{*} \omega_{Y}[m]
$$

из которых сразу следует, что размерности $n$ и $m$ многообразий $X$ и $Y$ равны.

Рассмотрим функтор

$$
\Phi_{\mathscr{F} \otimes \mathscr{E}}: \mathbf{D}^{b}(X \times X) \longrightarrow \mathbf{D}^{b}(Y \times Y)
$$

и обозначим его как $A d_{\mathscr{E}}$. Функтор $A d_{\mathscr{E}}$ также является эквивалентностью по предложению 2.1.7. Кроме того, по предложению 2.1.6 для любого объекта $\mathscr{G} \in \mathbf{D}^{b}(X \times X)$ сушествует изоморфизм функторов:

$$
\Phi_{A d_{\mathscr{E}}(\mathscr{G})} \cong \Phi_{\mathscr{E}} \circ \Phi_{\mathscr{G}} \circ \Phi_{\mathscr{E}}^{-1}
$$

Рассмотрим специальный случай, когда объект $\mathscr{G}$ есть структурный пучок диагонали $\mathscr{O}_{\Delta_{X}}$. Он представляет тождественньй функтор. Следовательно, применяя формулу (12), получаем, что функтор $A d_{\mathscr{E}}$ переводит структурный пучок диагонали $\mathscr{O}_{\Delta_{X}}$ в структурньй пучок диагонали $\mathscr{O}_{\Delta_{Y}}$. 
Рассмотрим более общую ситуацию. Обозначим через $i_{X}$ и $i_{Y}$ вложения диагоналей в $X \times X$ и $Y \times Y$ соответственно. Применим функтор $A d_{\mathscr{E}}$ к объекту $i_{X *} \omega_{X}^{k}$, где $\omega_{X}$, как и раньше, канонический пучок на $X$. Объект $i_{X} \omega_{X}^{k}$ представляет функтор $S^{k}[-n k]$, где $S$ - функтор Серра в категории $\mathbf{D}^{b}(X)$. Так как любая эквивалентность перестановочна с функторами Серра по лемме 1.1.4, получаем, что

$$
A d_{\mathscr{E}}\left(i_{X *} \omega_{X}^{k}\right) \cong i_{Y *} \omega_{Y}^{k}
$$

Определим теперь для каждого многообразия $X$ биградуированную алгебру

$$
\mathrm{HA}(X)=\bigoplus_{i, k} \mathrm{HA}_{i, k}(X):=\bigoplus_{i, k} \operatorname{Ext}_{X \times X}^{i}\left(\mathscr{O}_{\Delta_{X}}, i_{X *} \omega_{X}^{k}\right)
$$

Структура алгебры здесь задается композицией Еxt'ов с учетом канонического отождествления

$$
\operatorname{Ext}_{X \times X}^{i}\left(\mathscr{O}_{\Delta_{X}}, i_{X *} \omega_{X}^{k}\right) \cong \operatorname{Ext}_{X \times X}^{i}\left(i_{X *} \omega^{m}, i_{X *} \omega_{X}^{m+k}\right)
$$

Для доказательства следуюшей теоремы нам придется применить основной результат третьей главы, утверждающий, что любая эквивалентность представляется объектом на произведении.

Теорема 2.1.8. Пусть $X$ и $Y$ - гладкие проективные многообразия, для которых производные категории когерентных пучков әквивалентны как триангулированные категории. Тогда биградуированные алгебры $\mathrm{HA}(X)$ и $\mathrm{HA}(Y)$ изоморфны.

ДокАЗАТЕльство. По теореме 3.2.2 всякая эквивалентность $F: \mathbf{D}^{b}(X) \rightarrow \mathbf{D}^{b}(Y)$ представляется некоторым объектом на произведении, т.е. изоморфна функтору $\Phi_{\mathscr{E}}$ для некоторого $\mathscr{E} \in \mathbf{D}^{b}(X \times Y)$. Любая такая эквивалентность задает эквивалентность

$$
A d_{\mathscr{E}}: \mathbf{D}^{b}(X \times X) \longrightarrow \mathbf{D}^{b}(Y \times Y)
$$

которая переводит объект $i_{X *} \omega_{X}^{k}$ в объект $i_{Y *} \omega_{Y}^{k}$. Эквивалентность $A d_{\mathscr{E}}$ индуцирует изоморфизмы

$$
\operatorname{Ext}_{X \times X}^{i}\left(\mathscr{O}_{\Delta_{X}}, i_{X *} \omega_{X}^{k}\right) \cong \operatorname{Ext}_{Y \times Y}^{i}\left(\mathscr{O}_{\Delta_{Y}}, i_{Y *} \omega_{Y}^{k}\right)
$$

и, следовательно, изоморфизм биградуированных алгебр $\mathrm{HA}(X)$ и $\mathrm{HA}(Y)$.

Стоит отметить, что из биградуированной алгебры НА $(X)$ можно получить каноническую и антиканоническую алгебры многообразия $X$. Действительно,

$$
\bigoplus_{k \geqslant 0} \mathrm{H}^{0}\left(X, \omega_{X}^{k}\right)=\bigoplus_{k \geqslant 0} \mathrm{HA}_{0, k}(X) \text { и } \bigoplus_{k \leqslant 0} \mathrm{H}^{0}\left(X, \omega_{X}^{k}\right)=\bigoplus_{k \leqslant 0} \mathrm{HA}_{0, k}(X) .
$$

Таким образом, из теоремы 2.1.8 получается следствие. 
СлЕДСТВИЕ 2.1.9. Если производные категории когерентных пучков двух гладких проективных многообразий $X$ и $Y$ әквивалентны, то канонические ( и антиканонические) алгебры многообразий $X$ и $Y$ изоморфны.

Данное следствие очень близко по своей формулировке теореме 2.1.3. Однако надо отметить, что доказательство теоремы 2.1.3, данное в статье [8], не опирается на основной результат следующей главы и, более того, явлется конструктивньм. Кроме этого, в теореме 2.1.3 не предполагается, что канонический (или антиканонический) пучок второго многообразия $X^{\prime}$ является обильньм - это получается из доказательства теоремы.

Можно также описать и все остальные пространства $\mathrm{HA}_{i, k}(X)$. В статье [40] доказано, что спектральная последовательность, которая считает

$$
\operatorname{HA}_{i, k}(X)=\operatorname{Ext}^{i}\left(\mathscr{O}_{\Delta_{X}}, i_{X * \omega_{X}}\right)
$$

через когомологии ограничения $O_{\Delta_{X}}$ на диагональ, вырождается в члене $E_{2}$. В частности, имеются изоморфизмы

$$
\mathrm{HA}_{i, k}(X) \cong \bigoplus_{p+q=i} \mathrm{H}^{p}\left(X, \Lambda^{q} T_{X} \otimes \omega_{X}^{k}\right)
$$

где $T_{X}$ - касательное расслоение на $X$. Более того, данньй изоморфизм преврашается в изоморфизм алгебр, т.е.

$$
\mathrm{HA}(X) \cong \bigoplus_{i, k} \bigoplus_{p+q=i} \mathrm{H}^{p}\left(X, \Lambda^{q} T_{X} \otimes \omega_{X}^{k}\right)
$$

как биградуированные алгебры. Из данного равенства и теоремы 2.1.8получаем следствие.

СЛЕДСТВИЕ 2.1.10. Если производные категории когерентных пучков двух гладких проективных многообразий $X \quad$ и $Y$ әквивалентны, то имеются изоморфизмы пространств

$$
\bigoplus_{p+q=i} \mathrm{H}^{p}\left(X, \Lambda^{q} T_{X} \otimes \omega^{k}\right) \cong \bigoplus_{p+q=i} \mathrm{H}^{p}\left(Y, \Lambda^{q} T_{Y} \otimes \omega^{k}\right)
$$

В частности, получаются изоморфизмы

$$
\bigoplus_{p-q=i} \mathrm{H}^{p}\left(X, \Omega_{X}^{q}\right) \cong \bigoplus_{p-q=i} \mathrm{H}^{p}\left(Y, \Omega_{Y}^{q}\right)
$$

межсду вертикалями ромба Ходжа. 
ДокАЗАТЕЛЬСТВо. Изоморфизмы (15) сразу следуют из теоремы 2.1 .8 и равенства (14). Изоморфизмы (16) являются частным случаем (15) при $k=1$.

Изоморфизмы между вертикалями ромба Ходжа можно получить и другим способом. Предположим, что основное поле $k$ есть $\mathbb{C}$.

Для каждого элемента $\xi \in \mathrm{H}^{*}(X \times Y, \mathbb{Q})$ можно определить линейные отображения

$$
v_{\xi}: \mathrm{H}^{*}(X, \mathbb{Q}) \longrightarrow \mathrm{H}^{*}(Y, \mathbb{Q}), \quad w_{\xi}: \mathrm{H}^{*}(Y, \mathbb{Q}) \longrightarrow \mathrm{H}^{*}(X, \mathbb{Q})
$$

по формулам

$$
v_{\xi}(-)=q_{*}\left(\xi \cdot p^{*}(-)\right), \quad w_{\xi}(-)=p_{*}\left(\xi \cdot q^{*}(-)\right) .
$$

Для данных отображений можно написать формулу композиции, которая аналогична формуле (9) для композищии функторов. Пусть $X, Y, Z$ - три гладких полных многообразия и $\xi, \eta$ - элементы $\mathrm{H}^{*}(X \times Y, \mathbb{Q})$ и $\mathrm{H}^{*}(Y \times Z, \mathbb{Q})$ соответственно. Тогда композиция $v_{\eta} \circ v_{\xi}$ совпадает с отображением $v_{\zeta}$, где $\zeta \in \mathrm{H}^{*}(X \times Z, \mathbb{Q})$ задается формулой

$$
\zeta=p_{X Z *}\left(p_{Y Z}^{*}(\eta) \cup p_{X Y}^{*}(\xi)\right) .
$$

Каждому функтору вида $\Phi_{\mathscr{E}}: \mathbf{D}^{b}(X) \longrightarrow \mathbf{D}^{b}(Y)$ можно сопоставить линейное отображение $\varphi_{\mathscr{E}}: \mathrm{H}^{*}(X, \mathbb{Q}) \longrightarrow \mathrm{H}^{*}(Y, \mathbb{Q})$. Для этого определим элемент $\varepsilon \in \mathrm{H}(X \times Y, \mathbb{Q})$ по правилу

$$
\varepsilon=p^{*} \sqrt{t d_{X}} \cdot \operatorname{ch}(E) \cdot q^{*} \sqrt{t d_{Y}}
$$

где $t d_{X}$ и $t d_{Y}-$ классы Тодда многообразий $X$ и $Y$. Зададим отображения

$$
\begin{aligned}
& \varphi_{\mathscr{E}}(-):=v_{\varepsilon}(-)=q_{*}\left(\varepsilon \cdot p^{*}(-)\right) \\
& \psi_{\mathscr{E}}(-):=w_{\varepsilon}(-)=p_{*}\left(\varepsilon \cdot q^{*}(-)\right)
\end{aligned}
$$

Следующее предложение является прямьм следствием теоремы Римана-Роха в форме Гротендика.

ПРЕДЛОЖЕНИЕ 2.1.11. Предположиим, что функтор $\Phi_{\mathscr{E}}: \mathbf{D}^{b}(X) \longrightarrow \mathbf{D}^{b}(Z)$ является композицией $\Phi_{\mathscr{G}} \circ \Phi_{\mathscr{F}}$ для некоторых

$$
\Phi_{\mathscr{F}}: \mathbf{D}^{b}(X) \longrightarrow \mathbf{D}^{b}(Y), \quad \Phi_{\mathscr{G}}: \mathbf{D}^{b}(Y) \longrightarrow \mathbf{D}^{b}(Z)
$$

Тогда $\varphi_{\mathscr{E}}=\varphi_{\mathscr{G}} \circ \varphi_{\mathscr{F}}$

Отсюда сразу получаем следствие.

СЛЕДСТВИЕ 2.1.12. Если функтор $\Phi_{\mathscr{E}}: \mathbf{D}^{b}(X) \longrightarrow \mathbf{D}^{b}(Z)$ является әквивалентностью, то отображсение $\varphi_{\mathscr{E}}: \mathrm{H}^{*}(X, \mathbb{Q}) \longrightarrow \mathrm{H}^{*}(Y, \mathbb{Q})$ есть изоморфизм, его комлексификация индуцирует изоморфизмы (16) между вертикалями ромба Ходжа. 
ДоКАЗАТЕльство. Из предложения 2.1.11 следует, что функтор, квазиобратньй к $\Phi_{\mathscr{E}}$, индуцирует отображение, обратное к $\varphi_{\mathscr{E}}$. Кроме того, так как элемент $\varepsilon \in$ $\mathrm{H}^{*}(X \times Y, \mathbb{Q})$ по формуле $(18)$ соответствует алгебраическому шиклу, то легко проверяется, что комплексификация отображения $\varphi_{\mathscr{E}}$ сохраняет вертикали ромба Ходжа.

В заключение отметим также, что всякий функтор $\Phi_{\mathscr{E}}: \mathbf{D}^{b}(X) \longrightarrow \mathbf{D}^{b}(Y)$ индуцирует отображение $\overline{\Phi_{\mathscr{E}}}: K(X) \longrightarrow K(Y)$ между группами Гротендика $K(X)$ и $K(Y)$ категорий $\mathbf{D}^{b}(X)$ и $\mathbf{D}^{b}(Y)$. Рассмотрим отображение

$$
\operatorname{ch} \sqrt{t d_{X}}: K(X) \longrightarrow \mathrm{H}^{*}(X, \mathbb{Q}),
$$

которое сопоставляет элементу из $K(X)$ его характер Черна, умноженньй на корень из класса Тодда. Изпользуя теорему Римана-Роха, можно показать, что диаграмма

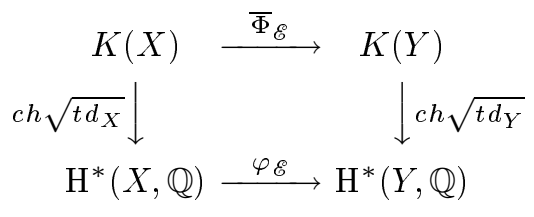

является коммутативной.

2.2. Примеры эквивалентностей: бирациональные преобразования типа флоп. В этом разделе приводится целый класс примеров двух гладких многообразий, для которых производные категории когерентных пучков эквивалентны. Конечно, примеры таких многообразий были уже известны: первый пример - это абелево многообразие и двойственное ему - был рассмотрен Мукаи (см. [29]). Принщипиальное отличие примеров, обсуждаемых в этом разделе, в том, что получаемая пара в обшем случае неизоморфных многообразий связана бирациональным изоморфизмом, которьй является флопом.

Из данных примеров также следует, что условия на (анти)канонический пучок в теореме 2.1.3 не могут быть ослаблены.

В начале раздела напомним определения допустимых подкатегорий и полуортогональных разложений (см. [5], [6]).

ОПРЕДЕЛЕНИЕ 2.2.1. Пусть $\mathscr{B}$ - полная аддитивная подкатегория в аддитивной категории $\mathscr{A}$. Правым ортогоналом к $\mathscr{B}$ в $\mathscr{A}$ назьвается полная подкатегория $\mathscr{B}^{\perp} \subset \mathscr{A}$, состоящая из всех таких объектов $C$, для которых $\operatorname{Hom}(B, C)=0$ для всех $B \in \mathscr{B}$. Двойственньм образом определяется левьй ортогонал $\perp \mathscr{B}$.

Отметим, что если $\mathscr{B}$ является триангулированной подкатегорией в триангулированной категории $\mathscr{A}$, то $\perp \mathscr{B}$ и $\mathscr{B}^{\perp}$ также являются триангулированньми подкатегориями.

ОПРЕДЕЛЕНИЕ 2.2.2. Пусть $I: \mathscr{N} \longrightarrow \mathscr{D}$ - вложение полной триангулированной подкатегории в триангулированную категорию $\mathscr{D}$. Будем говорить, что $\mathscr{N}$ доnустима справа (соответственно слева), если существует функтор $P: \mathscr{D} \longrightarrow \mathscr{N}$, сопряженньй справа (соответственно слева) к функтору вложения $I$.

Свойство быть допустимой справа (соответственно слева) для подкатегории $\mathcal{N}$ эквивалентно следующему свойству, которое формулируется в терминах ортогоналов: 
для каждого объекта $X \in \mathscr{D}$ сушествует выделенный треугольник $N \rightarrow X \rightarrow M$, где $N \in \mathscr{N}_{\text {и }} M \in \mathscr{N}^{\perp}$ (соответственно $M \rightarrow X \rightarrow N$, где $M \in{ }^{\perp} \mathscr{N}$ и $N \in \mathscr{N}$ ). Подкатегорию будем называть просто допустимой, если она допустима как справа, так и слева.

Если $\mathscr{N} \subset \mathscr{D}$ - допустимая подкатегория, то скажем, что $\mathscr{D}$ допускает полуортогональное разложение вида $\left\langle\mathscr{N}^{\perp}, \mathscr{N}\right\rangle$ или $\left\langle\mathscr{N},{ }^{\perp} \mathscr{N}\right\rangle$. Иногда бьвает, что такой процесс разложения можно продолжить дальше, раскладьвая подкатегорию $\mathscr{N}$ или ортогоналы к ней. Дадим общее определение полуортогонального разложения.

ОПРЕДЕЛЕНИЕ 2.2.3. Последовательность допустимых подкатегорий $\left(\mathscr{N}_{0}, \ldots\right.$, $\left.\mathscr{N}_{n}\right)$ в триангулированной категории $\mathscr{D}$ назьвается полуортогональной, если выполняется условие $\mathscr{N}_{j} \subset \mathscr{N}_{i}^{\perp}$ для всех $0 \leqslant j<i \leqslant n$. Полуортогональная последовательность назьвается полной, если она порождает категорию $\mathscr{D}$, то есть минимальная триангулированная подкатегория в $\mathscr{D}$, содержашая все $\mathscr{N}_{i}$, совпадает с $\mathscr{D}$. В этом случае такая последовательность называется полуортогональным разложением категории $\mathscr{D}$ и записьвается в виде:

$$
\mathscr{D}=\left\langle\mathscr{N}_{0}, \ldots, \mathscr{N}_{n}\right\rangle
$$

Простейший пример полуортогонального разложения возникает при наличии полного исключительного набора.

ОПРЕДЕЛЕНИЕ 2.2.4. Объект $E$ в триангулированной категории $\mathscr{D}$ назьвается $u c$ ключительным, если $\operatorname{Hom}^{i}(E, E)=0$ при $i \neq 0$ и $\operatorname{Hom}(E, E)=k$. Упорядоченный набор исключительных объектов $\left(E_{0}, \ldots, E_{n}\right)$ назьвается полным исключительным набором, если он порождает категорию $\mathscr{D}$ и $\operatorname{Hom}^{\cdot}\left(E_{i}, E_{j}\right)=0$ при $i>j$.

Самый известньй пример полного исключительного набора предоставляет проективное пространство.

ПримеР 2.2.5 [2]. На проективном пространстве $\mathbb{P}^{N}$ для каждого $i \in \mathbb{Z}$ набор вида $(\mathscr{O}(i), \ldots, \mathscr{O}(i+N))$ является исключительным и полньм. В частности, получается, что производная категория когерентных пучков $\mathbf{D}^{b}\left(\mathbb{P}^{N}\right)$ эквивалентна производной категории конечномерных модулей над конечномерной алгеброй эндоморфизмов исключительного набора $\operatorname{End}\left(\bigoplus_{j=0}^{N} \mathscr{O}(j)\right)$.

Аналогичные разложения существуют и для некоторых других многообразий таких как квадрики и многообразия флагов [20]-[22].

Теперь приведем необходимые нам факты о раздутиях и о поведении производной категории когерентных пучков при раздутиях. Все эти результаты содержатся в статье [34] (см. также [7]). Пусть $X$ - гладкое полное алгебраическое многообразие и $Y \subset X$ - гладко вложенное замкнутое подмногообразие коразмерности $r$. Обозначим через $\widetilde{X}$ раздутие $X$ с центром вдоль $Y$. Многообразие $\widetilde{X}$ является также гладким и сушествует коммутативная диаграмма:

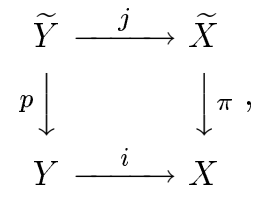


в которой $i$ и $j$ - замкнутые вложения и $p: \tilde{Y} \rightarrow Y$ - проективное расслоение исключительного дивизора $\widetilde{Y}$ над центром раздутия $Y$, в частности, $p$ - плоский морфизм. Напомним, что $\widetilde{Y} \cong \mathbb{P}\left(N_{X / Y}\right)$, где $N_{X / Y}$ - нормальное расслоение к $Y$ в $X$. Обозначим через $\mathscr{O}_{\widetilde{Y}}(1)$ каноническое относительно обильное линейное расслоение на $\tilde{Y}=\mathbb{P}\left(N_{X / Y}\right)$. Хорошо известно, что оно изоморфно ограничению линейного расслоения $\mathscr{O}(-\widetilde{Y})$ на $\tilde{Y}$.

ПРЕДЛОЖЕНИЕ 2.2.6 [34]. Производные функторы обратного образа

$$
\mathbf{L} \pi^{*}: \mathbf{D}^{b}(X) \longrightarrow \mathbf{D}^{b}(\tilde{X}), \quad p^{*}: \mathbf{D}^{b}(Y) \longrightarrow \mathbf{D}^{b}(\tilde{Y})
$$

являются вполне строгими.

ДокАЗАТЕЛЬСТво. Формула проекции (3) дает изоморфизм:

$$
\operatorname{Hom}\left(\mathbf{L} \pi^{*} F, \mathbf{L} \pi^{*} G\right) \cong \operatorname{Hom}\left(F, \mathbf{R} \pi_{*} \mathbf{L} \pi^{*} G\right) \cong \operatorname{Hom}\left(F, \mathbf{R} \pi_{*} \mathscr{O}_{\widetilde{Y}} \stackrel{\mathbf{L}}{\otimes} G\right),
$$

где $F, G \in \mathbf{D}^{b}(X)$. Аналогично и для $p^{*}$. Комбинируя их с тем фактом, что $\mathbf{R} \pi_{*} \mathscr{O}_{\widetilde{X}} \cong$ $\mathscr{O}_{X}$ и $\mathbf{R} p_{*} \mathscr{O}_{\widetilde{Y}}=\mathscr{O}_{Y}$, получаем доказательство.

ПРЕДЛОЖЕНИЕ 2.2.7 [34], [7]. Для всякого обратимого пучка $\mathscr{L}$ на $\widetilde{Y}$ функтор

$$
\mathbf{R} j_{*}\left(\mathscr{L} \otimes p^{*}(\cdot)\right): \mathbf{D}^{b}(Y) \longrightarrow \mathbf{D}^{b}(\widetilde{X})
$$

является вполне строгим.

ДокАЗАТЕльство. Чтобы доказать, что функтор вполне строгий, достаточно проверить вьполнение условий 1) и 2) теоремы 2.1.5. Для каждой замкнутой точки $y \in Y$ образ $\Phi\left(\mathscr{O}_{y}\right)$ является структурным пучком соответствующего слоя отображения $p$, рассматриваемым как пучок на $\widetilde{X}$. Так как слои над разными точками не пересекаются, то условие ортогональности 1 ) теоремы 2.1.5 вьполняется.

Рассмотрим структурный пучок $\mathscr{O}_{F}$ некоторого $p$-слоя $F \subset \widetilde{Y}$. Имеем изоморфизм:

$$
\operatorname{Hom}^{i}\left(j_{*} \mathscr{O}_{F}, j_{*} \mathscr{O}_{F}\right) \cong \operatorname{Hom}^{i}\left(\mathbf{L} j^{*} j_{*} \mathscr{O}_{F}, \mathscr{O}_{F}\right) .
$$

В производной категории $\mathbf{D}^{b}(\tilde{Y})$ имеется выделенньй треугольник

$$
\mathscr{O}_{F} \otimes \mathscr{O}_{\widetilde{Y}}(1)[1] \longrightarrow \mathbf{L} j^{*} j_{*} \mathscr{O}_{F} \longrightarrow \mathscr{O}_{F},
$$

где $\mathscr{O}_{\widetilde{Y}}(1)$ - это относительно обильное линейное расслоение на $\widetilde{Y}$, которое изоморфно $\left.\mathscr{O}(-\widetilde{Y})\right|_{\tilde{Y}}$. Слой $F$ есть проективное пространство, а ограничение пучка $\mathscr{O}_{\widetilde{Y}}(1)$ на $F$ изоморфно $\mathscr{O}(1)$. Поэтому

$$
\operatorname{Hom}^{i}\left(\mathscr{O}_{F} \otimes \mathscr{O}_{\widetilde{Y}}(1), \mathscr{O}_{F}\right)=0
$$

для всех $i$. И, значит,

$$
\operatorname{Hom}^{i}\left(j_{*} \mathscr{O}_{F}, j_{*} \mathscr{O}_{F}\right) \cong \operatorname{Hom}^{i}\left(\mathscr{O}_{F}, \mathscr{O}_{F}\right) .
$$

Следовательно, условие 2) теоремы 2.1.5 также вьполняется.

Обозначим через $D(X)$ полную триангулированную подкатегорию в $\mathbf{D}^{b}(\widetilde{X})$, которая является образом категории $\mathbf{D}^{b}(X)$ относительно функтора $\mathbf{L} \pi^{*}$, а через $D(Y)_{k}$ обозначим полную подкатегорию в $\mathbf{D}^{b}(\widetilde{X})$, которая является образом категории $\mathbf{D}^{b}(Y)$ относительно функтора $\mathbf{R} j_{*}\left(\mathscr{O}_{\widetilde{Y}}(k) \otimes p^{*}(\cdot)\right)$, где $\mathscr{O}_{\widetilde{Y}}(k)=\mathscr{O}_{\widetilde{Y}}(1)^{\otimes k}$ и $\mathscr{O}_{\widetilde{Y}}(1)-$ каноническое относительно обильное линейное расслоение на $Y=\mathbb{P}\left(N_{X / Y}\right)$. Из предложений 2.2.6 и 2.2.7 следует, что $D(X) \cong \mathbf{D}^{b}(X)$ и $D(Y)_{k} \cong \mathbf{D}^{b}(Y)$. 
ТеОРема 2.2.8 [34]. Последовательность допустимых подкатегорий

$$
\left\langle D(Y)_{-r+1}, \ldots, D(Y)_{-1}, D(X)\right\rangle
$$

является полуортогональной и дает полуортогональное разложение для категории $\mathbf{D}^{b}(\widetilde{X})$.

Эта теорема позволяет описать категорию раздутия в терминах многообразия, которое раздувается, и подмногообразия в котором раздуваем. Используя приведенное вьше описание производной категории раздутия, исследуем теперь поведение производной категории при простейших преобразованиях типа флип и флоп. Рассмотрим такой пример.

Пусть $Y$ - гладко вложенное замкнутое подмногообразие в гладком полном алгебраическом многообразии $X$ такое, что $Y \cong \mathbb{P}^{k}$ с нормальным расслоением $N_{X / Y} \cong$ $\mathscr{O}_{Y}(-1)^{\oplus(l+1)}$. Будем предполагать, что $l \leqslant k$.

Обозначим через $\widetilde{X}$ раздутие $X$ с центром вдоль $Y$. В данном случае исключительный дивизор $\tilde{Y}$ изоморфен произведению проективных пространств $\mathbb{P}^{k} \times \mathbb{P}^{l}$. Кроме того, в этой ситуации имеется следующее описание нормального пучка к $\widetilde{Y}$ в $\widetilde{X}$ :

$$
N_{\tilde{X} / \tilde{Y}}=\left.\mathscr{O}_{\tilde{X}}(\widetilde{Y})\right|_{\widetilde{Y}} \cong \mathscr{O}(-1 ;-1),
$$

где $\mathscr{O}(-1 ;-1):=p_{1}^{*} \mathscr{O}_{\mathbb{P} k}(-1) \otimes p_{2}^{*} \mathscr{O}_{\mathbb{P} l}(-1)$. Эти факты позволяют утверждать, что существует сдутие $\widetilde{X}$ такое, что $\widetilde{Y}$ проектируется на второй сомножитель $\mathbb{P}^{l}$. Это сдутие существует в аналитической категории, и его результат есть гладкое многообразие $X^{+}$, которое, вообще говоря, может быть не алгебраическим. Мы предположим, что оно алгебраическое. Вся геометрия, описанная вьше, отражена в следуюшей диаграмме:

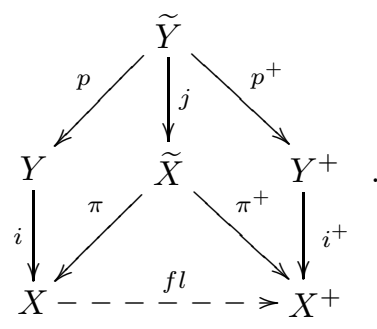

Бирашиональное отображение $f l: X \longrightarrow X^{+}$является простейшим примером преобразования типа флип-фолоп и есть флип при $l<k$ и фолоп при $l=k$.

В дальнейшем нам понадобится формула для ограничения канонического пучка $\omega_{\tilde{X}}$ на дивизор $\tilde{Y}$. При раздутии гладкого подмногообразия получаем

$$
\omega_{\widetilde{X}} \cong \pi^{*} \omega_{X} \otimes \mathscr{O}_{\widetilde{X}}(l \tilde{Y}) .
$$

Из формулы присоединения находим, что

$$
\left.\omega_{X}\right|_{Y} \cong \omega_{Y} \otimes \Lambda^{l+1} N_{X / Y}^{*} \cong \mathscr{O}_{Y}(l-k) .
$$

Комбинируя эти факты вместе, получаем изоморфизм:

$$
\left.\left.\left.\omega_{\widetilde{X}}\right|_{\widetilde{Y}} \cong\left(\pi^{*} \omega_{X} \otimes \mathscr{O}_{\widetilde{X}}(l \widetilde{Y})\right)\right|_{\widetilde{Y}} \cong p^{*}\left(\left.\omega_{X}\right|_{Y}\right) \otimes \mathscr{O}_{\widetilde{X}}(l \widetilde{Y})\right|_{\widetilde{Y}} \cong \mathscr{O}(-k ;-l) .
$$

Основная теорема этого раздела связьвает производные категории когерентных пучков на многообразиях $X$ и $X^{+}$. 
ТеОрема 2.2.9. Пусть $\mathscr{L}$ - линейное расслоение на $\widetilde{X}$. В обозначениях, введенных выше, функтор

$$
\mathbf{R} \pi_{*}\left(\mathbf{L} \pi^{+*}(\cdot) \otimes \mathscr{L}\right): \mathbf{D}^{b}\left(X^{+}\right) \longrightarrow \mathbf{D}^{b}(X)
$$

является вполне строгим.

ДокАЗАТЕльство. Вначале рассмотрим ограничение $\mathscr{L}$ на $\tilde{Y}$. Так как $\tilde{Y}=$ $\mathbb{P}^{k} \times \mathbb{P}^{l}$, то $\left.\mathscr{L}\right|_{\widetilde{Y}} \cong \mathscr{O}(a ; b)$ для некоторых цельх чисел $a$ и $b$.

Нужно показать, что для любой пары $A, B \subset \mathbf{D}^{b}\left(X^{+}\right)$сквозное отображение

$$
\begin{aligned}
\operatorname{Hom}(A, B) & \stackrel{\sim}{\longrightarrow} \operatorname{Hom}\left(\mathbf{L} \pi^{+*} A, \mathbf{L} \pi^{+*} B\right) \\
& \longrightarrow \operatorname{Hom}\left(\mathbf{R} \pi_{*}\left(\mathbf{L} \pi^{+*} A \otimes \mathscr{L}\right), \mathbf{R} \pi_{*}\left(\mathbf{L} \pi^{+*} B \otimes \mathscr{L}\right)\right)
\end{aligned}
$$

является изоморфизмом. Из сопряженности функторов имеем изоморфизм

$$
\begin{aligned}
& \operatorname{Hom}\left(\mathbf{R} \pi_{*}\left(\mathbf{L} \pi^{+*} A \otimes \mathscr{L}\right), \mathbf{R} \pi_{*}\left(\mathbf{L} \pi^{+*} B \otimes \mathscr{L}\right)\right) \\
& \quad \cong \operatorname{Hom}\left(\mathbf{L} \pi^{*} \mathbf{R} \pi_{*}\left(\mathbf{L} \pi^{+*} A \otimes \mathscr{L}\right), \mathbf{L} \pi^{+*} B \otimes \mathscr{L}\right) .
\end{aligned}
$$

Рассмотрим выделенный треугольник:

$$
\mathbf{L} \pi^{*} \mathbf{R} \pi_{*}\left(\mathbf{L} \pi^{+*} A \otimes \mathscr{L}\right) \longrightarrow \mathbf{L} \pi^{+*} A \otimes \mathscr{L} \longrightarrow \bar{A}
$$

Таким образом, чтобы доказать, что сквозное отображение (22) является изоморфизмом, необходимо и достаточно показать, что

$$
\operatorname{Hom}\left(\bar{A}, \mathbf{L} \pi^{+*} B \otimes \mathscr{L}\right)=0
$$

Так как композиция $\mathbf{R} \pi_{*} \mathbf{L} \pi^{*}$ изоморфна тождественному функтору по предложению 2.2.6, то, применяя функтор $\mathbf{R} \pi_{*}$ к выделенному треугольнику (23), получаем, что $\mathbf{R} \pi_{*} \bar{A}=0$. И, следовательно, $\operatorname{Hom}\left(\mathbf{L} \pi^{*} C, \bar{A}\right)=0$ для любого объекта $C \in$ $\mathbf{D}^{b}\left(X^{+}\right)$. Значит, объект $\bar{A}$ принадлежит подкатегории $D(X)^{\perp}$.

Из теоремы 2.2.8 следует полуортогональное разложение:

$$
D(X)^{\perp}=\left\langle D(Y)_{-l}, \ldots, D(Y)_{-1}\right\rangle .
$$

Так как $Y$ - это проективное пространство, то из примера 2.2.5 следует, что каждая подкатегория $D(Y)_{-i}$ обладает полным исключительным набором. Соединяя их вместе, получаем полньй исключительный набор в $D(X)^{\perp}$. Для нас будет удобен такой набор:

$$
\begin{aligned}
& D(X)^{\perp}=\left\langle\mathbf{R} j_{*} \mathscr{O}(a-k ;-l), \quad \ldots \quad \ldots \quad \mathbf{R} j_{*} \mathscr{O}(a ;-l),\right.
\end{aligned}
$$

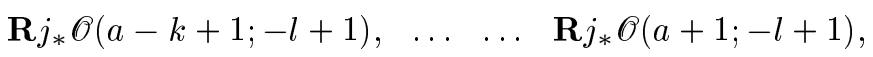

$$
\begin{aligned}
& \begin{array}{llll}
\cdots & \ldots & \ldots & \ldots \\
\mathbf{R} j_{*} \mathscr{O}(a-k+l-1 ;-1), & \ldots & \ldots & \left.\mathbf{R} j_{*} \mathscr{O}(a+l-1 ;-1)\right\rangle .
\end{array}
\end{aligned}
$$


Можно теперь перегруппировать эту исключительную последовательность таким образом, что для категории $D(X)^{\perp}$ получается полуортогональное разложение вида

$$
D(X)^{\perp}=\langle\mathscr{B}, \mathscr{A}\rangle,
$$

где $\mathscr{A}$ и $\mathscr{B}$ - подкатегории, порожденные $\mathbf{R} j_{*} \mathscr{O}(i ; s)$ с $i \geqslant a$ и $i<a$ соответственно. При $1 \leqslant i \leqslant k$ и $1 \leqslant s \leqslant l$ объекты $\mathbf{R} j_{*} \mathscr{O}(a-i ;-s)$ принадлежат одновременно подкатегориям $D(X)^{\perp}$ и $D\left(X^{+}\right)^{\perp} \otimes \mathscr{L}$. В частности, $\mathscr{B} \subset D(X)^{\perp} \cap\left(D\left(X^{+}\right)^{\perp} \otimes \mathscr{L}\right)$. Применяя функтор Нот к выделенному треугольнику (23), получаем, что

$$
\operatorname{Hom}\left(\bar{A}, \mathbf{R} j_{*} \mathscr{O}(a-i ;-s)\right)=0 \text { при } 1 \leqslant i \leqslant k \text { и } 1 \leqslant s \leqslant l .
$$

Так как $\bar{A} \in D(X)^{\perp}$ и ортогонален подкатегории $\mathscr{B}$, сразу следует, что $\bar{A} \in \mathscr{A}$. Теперь заметим, что если объект $\mathbf{R} j_{*} \mathscr{O}(a+i ; s)$ принадлежит подкатегории $\mathscr{A}$, то $i$ удовлетворяет неравенствам $0 \leqslant i<l$. Учитьвая формулу (21) для канонического класса $\left.\omega_{\tilde{X}}\right|_{\tilde{Y}} \cong \mathscr{O}(-k ;-l)$ и то, что $l \leqslant k$, получаем, что $\mathscr{A} \otimes \omega_{\tilde{X}} \subset D\left(X^{+}\right)^{\perp} \otimes \mathscr{L}$. Следовательно, для любого объекта $B \in \mathbf{D}^{b}\left(X^{+}\right)$

$$
\operatorname{Hom}\left(\mathbf{L} \pi^{+*} B \otimes \mathscr{L}, \bar{A} \otimes \omega_{\tilde{X}}\right)=0 .
$$

Применяя двойственность Серра (6), немедленно получаем необходимое равенство $\operatorname{Hom}\left(\bar{A}, \mathbf{L} \pi^{+*} B \otimes \mathscr{L}\right)=0$.

Tеорема 2.2.10. В тех же обозначениях, если $l=k$ (u, значит, fl ecть флоп) функтор $\mathbf{R} \pi_{*}\left(\mathbf{L} \pi^{+*}(\cdot) \otimes \mathscr{L}\right)$ является әквивалентностью триангулированных категорий.

ДокАЗАТЕЛЬСТвО. По предыдущей теореме данньй функтор является вполне строгим. Левый сопряженньй к нему имеет вид $\mathbf{R} \pi_{*}^{+}\left(\mathbf{L} \pi^{*}(\cdot) \otimes \mathscr{L}^{\prime}\right)$, где $\mathscr{L}^{\prime}=\mathscr{L}^{-1} \otimes$ $\omega_{\widetilde{X}} \otimes \pi^{+*} \omega_{X^{+}}^{-1} \cdot$ И, значит, по предыдушей теореме он также является вполне строгим. Что доказывает, что оба функтора суть эквивалентности.

Отметим, что доказательство данных утверждений проходит также и в случае, когда флоп делается сразу в некотором конечном множестве непересекающихся подмногообразий $Y_{1}, \ldots, Y_{s}$, каждое из которых удовлетворяет условию теорем. Это простое замечание является сушественным в связи с нашим предположением об алгебраичности получившегося многообразия $X^{+}$. Дело в том, что в примерах часто возникают ситуации, когда такие бирациональные преобразования, сделанные в одном из подмногообразий $Y_{i}$, приводят к неалгебраическим многообразиям, в то время как флип (или флоп), сделанный одновременно во всем наборе, дает многообразие, которое уже алгебраическое.

Второе замечание состоит в том, что, конечно, флопируемые многообразия $X$ и $X^{+}$ из теоремы 2.2.10 в обшем случае не изоморфны и часто встречаются в бирациональной геометрии, например, в конструкции при описании трехмерных многообразий Фано, которая называется двойная проекция из прямой (см. [19; §8]). Предположим, что имеется трехмерное многообразие Фано $V$ индекса 1 с Pic $V=\mathbb{Z}$, вложенное антиканонической системой в проективное пространство. Тогда раздутие этого многообразия в прямой дает многообразие $X$, антиканонический класс которого “почти” обилен отображение антиканонической системой стягивает некоторый набор кривых на этом 
многообразии, которые являются собственными прообразами прямых на $V$, пересекающих раздуваемую прямую. Во многих примерах эти кривые имеют нормальный пучок $\mathscr{O}(-1) \oplus \mathscr{O}(-1)$, и, следовательно, мы оказьваемся в ситуации нашей теоремы. Делая одновременный флоп в этих кривых, получаем многообразие $X^{+}$, которое неизоморфно $X$, но по теореме 2.2.10 имеет такую же производную категорию когерентных пучков. В частности, этот пример показывает, что условие обильности на антиканонический класс в теореме 2.1.3 о восстановлении не может быть ослаблено. Аналогичные примеры существуют и для многообразий общего типа и возникают в программе минимальных моделей. Данные результаты имеют еще одно также естественное обобщение. Предположим, что гладкое подмногообразие $Y$ в гладком полном алгебраическом многообразии $X$ является проективизацией векторного расслоения $E$ ранга $k+1$ над гладким многообразием $Z$, т.е. $Y \cong \mathbb{P}(E) \longrightarrow Z$. Допустим также, что нормальное расслоение $N_{X / Y}$ при ограничении на слой отображения $Y \longrightarrow Z$ изоморфен $\mathscr{O}_{\mathbb{P} k}(-1)^{\oplus(l+1)}$. Будем опять предполагать, что $l \leqslant k$.

Обозначая через $\widetilde{X}$ раздутие $X$ с центром вдоль $Y$, снова получаем диаграмму вида $(20)$, где $Y^{+}$является проективизацией некоторого расслоения ранга $l+1$ над $Z$. В данной ситуации можно утверждать, что аналоги теорем 2.2.9 и 2.2.10 также верны.

Другие похожие примеры возникают в случае, когда $X$ - трехмерное многообразие, а $Y$ - рациональная кривая, для которой выполнено $Y \cdot K_{X}=0$. В этом случае нормальное расслоение к $Y$ может иметь вид $\mathscr{O}(-1) \oplus \mathscr{O}(-1), \mathscr{O} \oplus \mathscr{O}(-2)$ или $\mathscr{O}(1) \oplus O(-3)$. Во всех этих случаях существует бирациональное преобразование типа флоп $f l: X \rightarrow X^{+}$. И во всех этих случаях производные категории когерентных пучков $X$ и $X^{+}$эквивалентны. Первый случай является частньм случаем теоремы 2.2.10. Второй вариант был рассмотрен в статье [7]. И недавно эквивалентность категорий была доказана для всех этих случаев вместе в статье [10].

\section{Глава 3. Вполне строгие функторы между производными категориями}

3.1. Диаграммы Постникова и их свертки. В этом разделе рассматриваются диаграммы Постникова в триангулированных категориях и находятся условия, при которых диаграмма Постникова имеет свертку и эта свертка однозначно определена.

Пусть $X^{\cdot}=\left\{X^{c} \stackrel{d^{c}}{\longrightarrow} X^{c+1} \stackrel{d^{c+1}}{\longrightarrow} \cdots \longrightarrow X^{0}\right\}$, где $c<0$ - ограниченньй комплекс объектов в триангулированной категории $\mathscr{D}$. Это значит, что все композиции $d^{i+1} \circ d^{i}$ равны 0.

Левая система Постникова, связанная с $X^{\bullet}$, есть по определению диаграмма следующего вида:

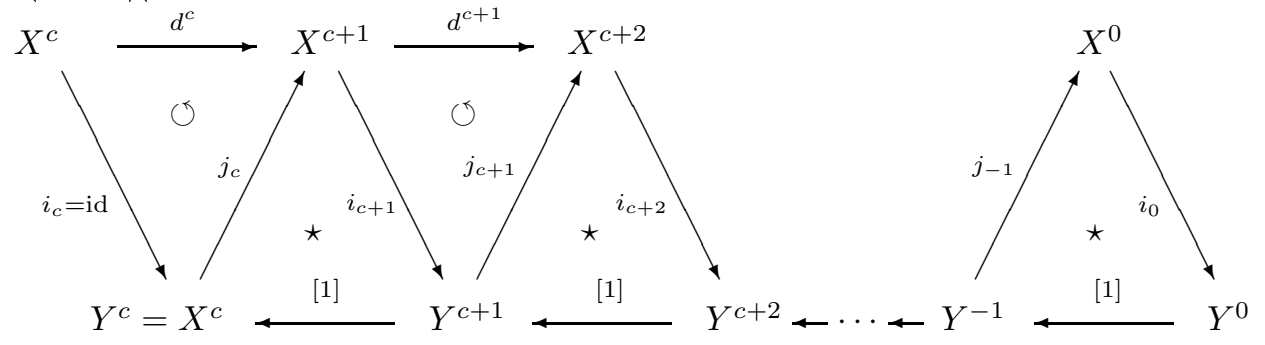

в которой все треугольники, отмеченные $\star$, являются выделенными, а все треугольники, отмеченные с помошњю $\circlearrowleft$, коммутативны (т.е. $j_{k} \circ i_{k}=d^{k}$ ). Объект $E \in \mathrm{Ob} \mathscr{D}$ 
называется левой сверткой комплекса $X^{*}$, если существует левая система Постникова, связанная с $X^{*}$, такая, что $E=Y^{0}$. Класс всех сверток комплекса $X^{\cdot}$ обозначим через $\operatorname{Tot}\left(X^{\cdot}\right)$. Очевидно, что системы Постникова и их свертки стабильны относительно точных функторов между триангулированными категориями.

Отметим, что класс $\operatorname{Tot}\left(X^{\cdot}\right)$ может содержать много неизоморфных объектов и может также быть пустьм. В дальнейшем будет описано условие, достаточное для того, чтобы класс $\operatorname{Tot}\left(X^{*}\right)$ состоял из одного объекта с точностью до изоморфизма. Следующая лемма доказана в [3].

Лемма 3.1.1. Пусть $g$ - морфизм междуу обгектами $Y$ и $Y^{\prime}$, которые, в свою очередь, включены в вылдленные треугольники:

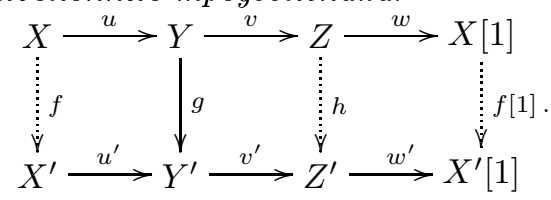

Eсли $v^{\prime} g u=0$, то существуют морфизмы $f: X \rightarrow X^{\prime}$ и $h: Z \rightarrow Z^{\prime}$ такие, что тройка $(f, g, h)$ является морфизмом треугольников.

Eсли, в дополнение, $\operatorname{Hom}\left(X[1], Z^{\prime}\right)=0$, тогда морфизмы $f u h$, делающие коммутативными первый и соответственно второй квадрат этой диаграммы, однозначно определень әтими условиями.

Теперь докажем две леммы, которые являются обобщением предыдущей для диаграмм Постникова.

ЛЕмма 3.1.2. Пусть $X^{\cdot}=\left\{X^{c} \stackrel{d^{c}}{\longrightarrow} X^{c+1} \stackrel{d^{c+1}}{\longrightarrow} \cdots \longrightarrow X^{0}\right\}-$ ограниченный комплекс оббектов в триангулированной категории $\mathscr{D}$. Предположим, что он удовлетворяет следующему условию:

$$
\operatorname{Hom}^{i}\left(X^{a}, X^{b}\right)=0 \quad \partial \Omega_{s} \quad i<0 \quad u \quad a<b .
$$

Тогда существует свертка этого комплекса и все свертки изоморфны друг другу (неканонически).

Если, кроме того,

$$
\operatorname{Hom}^{i}\left(X^{a}, Y^{0}\right)=0 \quad \text { для } i<0 \quad \text { u } \quad \text { всех } \quad \text { a }
$$

для некоторой свертки $Y^{0}$ ( и, следовательно, для любой свертки), тогда все свертки этого комплекса канонически изоморфны друг другу.

ЛЕмма 3.1.3. Пусть $X_{1}$ и $X_{2}^{\dot{2}}$ - ограниченные комплексы, которые удовлетворяют условию (25), и пусть $\left(f_{c}, \ldots, f_{0}\right)$ - морфизм межсду этими комплексами:

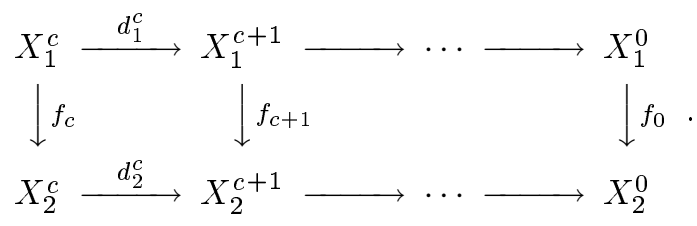


Предположим, что

$$
\operatorname{Hom}^{i}\left(X_{1}^{a}, X_{2}^{b}\right)=0 \quad \partial \Omega_{я} \quad i<0 \quad u \quad \partial \Omega_{\Omega} \quad a<b .
$$

Тогда для каждой свертки $Y_{1}^{0}$ комплекса $X_{1}$ и для каждой свертки $Y_{2}^{0}$ комплекса $X_{2}$ существует морфизм $f: Y_{1}^{0} \rightarrow Y_{2}^{0}$, который коммутирует с морфизмом $f_{0}$. Если кроме того

$$
\operatorname{Hom}^{i}\left(X_{1}^{a}, Y_{2}^{0}\right)=0 \quad \text { для } i<0 \quad \text { u } \quad \text { всех } \quad a
$$

тогда этот морфизм однозначно определен.

ДокАЗАТЕльство. Будем доказывать обе леммы вместе. Доказательство будем вести по индукции, а основанием индукции является лемма 3.1.1. Пусть $Y^{c+1}-$ конус морфизма $d^{c}$ :

$$
X^{c} \stackrel{d^{c}}{\longrightarrow} X^{c+1} \stackrel{\alpha}{\longrightarrow} Y^{c+1} \longrightarrow X^{c}[1] .
$$

По условию $d^{c+1} \circ d^{c}=0$ и $\operatorname{Hom}\left(X^{c}[1], X^{c+2}\right)=0$. Следовательно, сушествует единственный морфизм $\bar{d}^{c+1}: Y^{c+1} \rightarrow X^{c+2}$ такой, что $\bar{d}^{c+1} \circ \alpha=d^{c+1}$.

Рассмотрим композицию $d^{c+2} \circ \bar{d}^{c+1}: Y^{c+1} \rightarrow X^{c+3}$. Известно, что $d^{c+2} \circ \bar{d}^{c+1} \circ \alpha=$ $d^{c+2} \circ d^{c+1}=0$, и, кроме того, имеется равенство $\operatorname{Hom}\left(X^{c}[1], X^{c+3}\right)=0$. Отсюда немедленно следует, что композиция $d^{c+2} \circ \bar{d}^{c+1}$ также равна 0 .

Рассматривая выделенньй треугольник (29), получаем, что

$$
\operatorname{Hom}^{i}\left(Y^{c+1}, X^{b}\right)=0
$$

при $i<0$ и $b>c+1$. Таким образом, комплекс $Y^{c+1} \longrightarrow X^{c+2} \longrightarrow \cdots \longrightarrow X^{0}$ также удовлетворяет условию (25). По предположению индукции он имеет свертку. Значит, комплекс $X^{\bullet}$ также обладает сверткой, и, следовательно, класс $\operatorname{Tot}\left(X^{\bullet}\right)$ не пуст.

Теперь покажем, что при условии (27) всякий морфизм комплексов может быть продолжен до морфизма систем Постникова.

Рассмотрим конусы $Y_{1}^{c+1}$ и $Y_{2}^{c+1}$ морфизмов $d_{1}^{c}$ и $d_{2}^{c}$. Сушествует морфизм $g_{c+1}: Y_{1}^{c+1} \rightarrow Y_{2}^{c+1}$, который дополняет пару $\left(f_{c}, f_{c+1}\right)$ до морфизма треугольников:

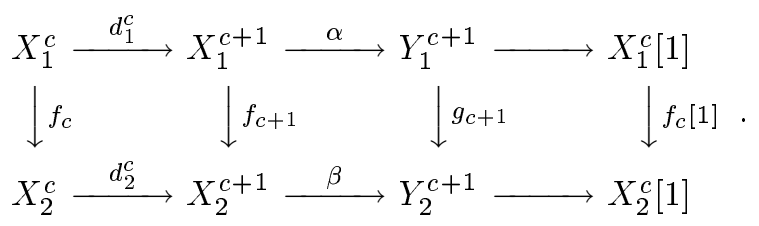

Как было показано вьше, сушествуют однозначно определенные морфизмы $\bar{d}_{i}^{c+1}: Y_{i}^{c+1} \rightarrow X_{i}^{c+2}$ для $i=1,2$. Рассмотрим следующую диаграмму:

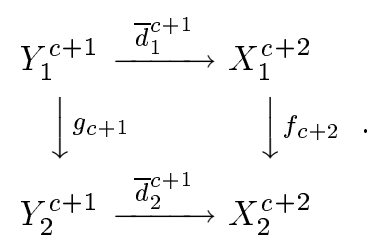


Докажем, что этот квадрат коммутативен. Действительно, обозначим через $h$ разность $f_{c+2} \circ \bar{d}_{1}^{c+1}-\bar{d}_{2}^{c+1} \circ g_{c+1}$. Имеется равенство $h \circ \alpha=f_{c+2} \circ d_{1}^{c+1}-d_{2}^{c+1} \circ f_{c+1}=0$. А по условию леммы $\operatorname{Hom}\left(X_{1}^{c}[1], X_{2}^{c+2}\right)=0$. Отсюда сразу следует, что $h=0$.

Таким образом, получается морфизм комплексов:

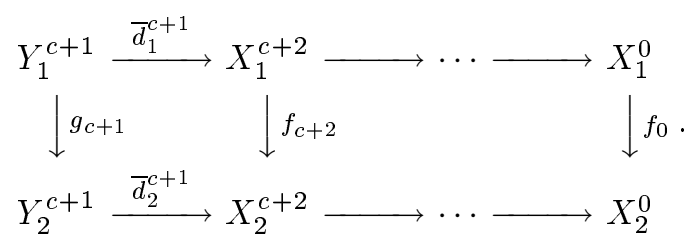

Эти комплексы удовлетворяют условиям (25) и (27). По индуктивному предположению морфизм между этими комплексами продолжается до морфизма систем Постникова. Следовательно, получаем морфизм между системами Постникова, связанными с комплексами $X_{\dot{1}}$ и $X_{2}$.

Кроме того, видно, что если все $f_{i}$ - изоморфизмы, то и морфизм между системами Постникова также является изоморфизмом. Следовательно, при условии (25) все объекты из $\operatorname{Tot}\left(X^{*}\right)$ изоморфны.

В заключение рассмотрим морфизм между выделенными треугольниками, которые являются частью диаграмм Постникова:

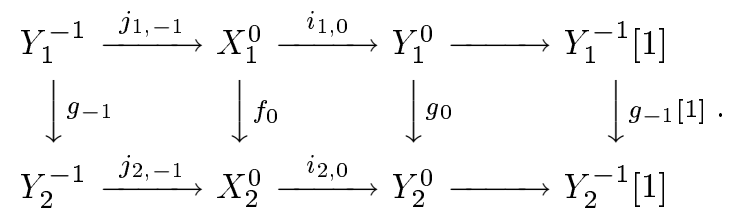

Если комплексы $X_{i}$ удовлетворяют условию (28) (т.е. $\operatorname{Hom}^{i}\left(X_{1}^{a}, Y_{2}^{0}\right)=0$ при $i<0$ и всех $a$ ), то получаем $\operatorname{Hom}\left(Y_{1}^{-1}[1], Y_{2}^{0}\right)=0$. И по лемме 3.1.1 морфизм $g_{0}$ однозначно определен. Леммы доказаны.

3.2. Вполне строгие функторы между производными категориями когерентных пучков. Пусть $X$ и $M$ - два гладких полных многообразия на- некоторым полем $k$. Как и раншше, будем обозначать через $\mathbf{D}^{b}(X)$ и $\mathbf{D}^{b}(M)$ ограниченные производные категории когерентных пучков на $X$ и $M$ соответственно, которые как было показано вьше, имеют структуру триангулированных категорий.

Рассмотрим произведение $M \times X$ и обозначим через $p$ и $\pi$ проекции $M \times X$ на $M$ и соответственно на $X$ :

$$
M \stackrel{p}{\stackrel{1}{\longleftarrow}} M \times X \stackrel{\pi}{\longrightarrow} X .
$$

Для каждого объекта $\mathscr{E} \in \mathbf{D}^{b}(M \times X)$ был определен точный функтор $\Phi_{\mathscr{E}}$ из $\mathbf{D}^{b}(M)$ в $\mathbf{D}^{b}(X)$ по формуле (7):

$$
\Phi_{\mathscr{E}}(\cdot):=\mathbf{R} \pi_{*}\left(\mathscr{E} \stackrel{\mathbf{L}}{\otimes} p^{*}(\cdot)\right) .
$$

$\Phi$ унктор $\Phi_{\mathscr{E}}$ имеет левьй и правьй сопряженные функторы $\Phi_{\mathscr{E}}^{*}$ и $\Phi_{\mathscr{E}}^{!}$соответственно, которые задавались формулами (8):

$$
\begin{aligned}
& \Phi_{\mathscr{E}}^{*}(\cdot)=\mathbf{R} p_{*}\left(\mathscr{E}^{\vee} \stackrel{\mathbf{L}}{\otimes} \pi^{*}\left(\omega_{X}[\operatorname{dim} X] \otimes(\cdot)\right)\right), \\
& \Phi_{\mathscr{E}}^{!}(\cdot)=\omega_{M}[\operatorname{dim} M] \otimes \mathbf{R} p_{*}(\mathscr{E} \vee \stackrel{\mathbf{L}}{\otimes}(\cdot)),
\end{aligned}
$$

где $\omega_{X}$ и $\omega_{M}-$ канонические пучки на $X$ и $M$ и $\mathscr{E} \vee:=\mathbf{R} \cdot \underline{\mathscr{H} o m}\left(\mathscr{E}, \mathscr{O}_{M \times X}\right)$. 
Чтобы исследовать вопрос, когда два многообразия имеют эквивалентные производные категории когерентных пучков, и описывать их группы автоэквивалентностей, желательно иметь явные формулы для всех точных функторов. Существует гипотеза, что все они представляются объектами на произведении, т.е. имеют вид (30). Однако до сих пор не известно, верно данное утверждение или нет. Тем не менее, оказывается, что частный случай этой гипотезы имеет место. А именно, если функтор является вполне строгим и имеет сопряженньй, то он может быть представлен объектом на произведении. Доказательству этого факта и посвящена данная глава. Более точно, основная теорема этой главы выглядит так.

TеОрема 3.2.1. Пусть $F-$ точныц функтор из категории $\mathbf{D}^{b}(M)$ в категорию $\mathbf{D}^{b}(X)$, где $M$ и $X$ гладкие проективные многообразия. Предположим, что $F$ является вполне строгим и имеет правый (или, соответственно, левый) сопряженный функтор. Тогда существует обгект $\mathscr{E} \in \mathbf{D}^{b}(M \times X)$ такой, что функтор $F$ изоморфен функтору $\Phi_{\mathscr{E}}$, определенному по правилу (30), и этот обгект однозначно определен с точностью до изоморфизма.

Отсюда немедленно получается, что всякая эквивалентность представляется объектом на произведении, так как любая эквивалентность имеет сопряженную, которая совпадает с квазиобратньм функтором.

ТеОРема 3.2.2. Пусть $M$ и $X-$ два гладких проективных многообразия. Предположим, что точный функтор $F: \mathbf{D}^{b}(M) \stackrel{\sim}{\longrightarrow} \mathbf{D}^{b}(X)$ является әквивалентностью триангулированных категорий. Тогда существует и единственныи $c$ точностью до изоморфизма облект $\mathscr{E} \in \mathbf{D}^{b}(M \times X)$ такой, что функтор $F$ изоморфен функтору $\Phi_{\mathscr{E}}$.

Эти результаты дают возможность описьвать все эквивалентности между производньми категориями когерентных пучков и отвечать на вопрос, когда два разных многообразия имеют эквивалентные производные категории когерентных пучков.

Прежде чем перейти к доказательству этих теорем, сделаем одно замечание. Пусть $F$ - точньй функтор из категории $\mathbf{D}^{b}(M)$ в категорию $\mathbf{D}^{b}(X)$. Обозначим через $F^{*}$ и $F^{!}$левьй и, соответственно, правый производные функторы, если они сушествуют. Если сушествует левьй сопряженньй $F^{*}$, тогда существует и правый сопряженньй $F^{!}$и он определяется формулой

$$
F^{!}=S_{M} \circ F^{*} \circ S_{X}^{-1}
$$

где $S_{X}$ и $S_{M}$ - функторы Серра в категориях $\mathbf{D}^{b}(X)$ и $\mathbf{D}^{b}(M)$, которые существуют и равны $(\cdot) \otimes \omega_{X}[\operatorname{dim} X]$ и, соответственно, $(\cdot) \otimes \omega_{M}[\operatorname{dim} M]($ см. (6)).

Пусть $F$ - точньй функтор из производной категории $\mathbf{D}^{b}(\mathscr{A})$ в производную категорию $\mathbf{D}^{b}(\mathscr{B})$. Будем говорить, что функтор $F$ ограничен, если существуют $z \in \mathbb{Z}$, $n \in \mathbb{N}$ такие, что для любого объекта $A \in \mathscr{A}$ когомологии $H^{i}(F(A))$ равны 0 при $i \notin[z, z+n]$.

Лемма 3.2.3. Пусть $M$ и $X$ - проективные многообразия и $M$ - гладкое многообразие. Если точный функтор $F: \mathbf{D}^{b}(M) \longrightarrow \mathbf{D}^{b}(X)$ имеет левый сопряэсенньй, тогда он ограничен. 
ДокАЗАТЕльство. Обозначим через $G: \mathbf{D}^{b}(X) \longrightarrow \mathbf{D}^{b}(M)$ левый сопряженный к $F$. Зафиксируем очень обильное линейное расслоение $\mathscr{L}$ на $X$. Оно задает вложение $i: X \hookrightarrow \mathbb{P}^{N}$. Для любого $k<0$ имеется правая резольвента пучка $\mathscr{O}(k)$ на $\mathbb{P}^{N}$ в терминах пучков $\mathscr{O}(j)$, где $j=0,1, \ldots, N$, которая имеет вид

$$
\mathscr{O}(k) \stackrel{\sim}{\longrightarrow}\left\{V_{0} \otimes \mathscr{O} \longrightarrow V_{1} \otimes \mathscr{O}(1) \longrightarrow \cdots \longrightarrow V_{N} \otimes \mathscr{O}(N) \longrightarrow 0\right\}
$$

где все $V_{j}$ - векторные пространства [2]. Ограничение этой резольвенты на $X$ дает резольвенту пучка $\mathscr{L}^{k}$ в терминах пучков $\mathscr{L}^{j}$, где $j=0,1, \ldots, N$. Так как для всех $j=0,1, \ldots, N$ ненулевые когомологии объектов $G\left(\mathscr{L}^{j}\right)$ принадлежат некоторому отрезку, то найдутся целое $z^{\prime}$ и натуральное $n^{\prime}$ такие, что для всех $k \leqslant 0$ когомологии $H^{l}\left(G\left(\mathscr{L}^{k}\right)\right)$ равны 0 при $l \notin\left[z^{\prime}, z^{\prime}+n^{\prime}\right]$. Это сразу следует из сушествования спектральной последовательности

$$
E_{1}^{p, q}=V_{p} \otimes H^{q}\left(G\left(\mathscr{L}^{p}\right)\right) \Rightarrow H^{p+q}\left(G\left(\mathscr{L}^{i}\right)\right) .
$$

Пусть $A \in \mathbf{D}^{b}(M)$ - некоторый объект. Из обильности расслоения $\mathscr{L}$ следует, что если для фиксированного $j$ имеем $\operatorname{Hom}^{j}\left(\mathscr{L}^{i}, F(A)\right)=0$ для всех $i \ll 0$, тогда когомология $H^{j}(F(A))$ равна 0 . По предположению функтор $G$ является левым сопряженным к $F$. Следовательно,

$$
\operatorname{Hom}^{j}\left(\mathscr{L}^{i}, F(A)\right) \cong \operatorname{Hom}^{j}\left(G\left(\mathscr{L}^{i}\right), A\right) .
$$

Рассмотрим теперь пучок $\mathscr{F}$ на многообразии $M$. Так как когомологии объектов $G\left(\mathscr{L}^{i}\right)$ для всех $i<0$ сосредоточены на отрезке $\left[z^{\prime}, z^{\prime}+n^{\prime}\right]$, то $\operatorname{Hom}^{j}\left(G\left(\mathscr{L}^{i}\right), \mathscr{F}\right)=0$ для всех $i<0$ и $j \notin\left[-z^{\prime}-n^{\prime},-z^{\prime}+\operatorname{dim} M\right]$ (здесь был использован тот факт, что гомологическая размерность категории $\operatorname{coh}(M)$ равна $\operatorname{dim} M)$. Следовательно, при тех же значениях $j$ имеем, что $H^{j}(F(\mathscr{F}))=0$ для любого пучка $\mathscr{F}$. И, значит, функтор $F$ ограничен.

ЗАмечАнИЕ 3.2.4. Заменяя, если необходимо, функтор $F$ при помоши сдвига в производной категории, с этого момента и на протяжении всей главы будем предполагать, что для любого пучка $\mathscr{F}$ на $M$ когомологии $H^{i}(F(\mathscr{F}))$ являются ненулевыми только при $i \in[-a, 0]$, где $a-$ некоторое фиксированное натуральное число.

3.3. Построение объекта, представляющего вполне строгий функтор. В этом разделе по точному вполне строгому функтору $F$ строится некоторый объект $\mathscr{E} \in \mathbf{D}^{b}(M \times X)$, а в следующем разделе будет доказано, что функторы $F$ и $\Phi_{\mathscr{E}}$ изоморфны. Построение объекта происходит в несколько этапов. Вначале рассматривается некоторое замкнутое вложение $j: M \hookrightarrow \mathbb{P}^{N}$ и строится некоторый объект $\mathscr{E}^{\prime} \in$ $\mathbf{D}^{b}\left(\mathbb{P}^{N} \times X\right)$. Затем показьвается, что на самом деле этот объект приходит с подмногообразия $M \times X$, т.е. сушествует объект $\mathscr{E} \in \mathbf{D}^{b}(M \times X)$ такой, что $\mathscr{E}^{\prime}=\mathbf{R} J_{*} \mathscr{E}$, где $J=(j \times \mathrm{id})$ - замкнутое вложение $M \times X$ в $\mathbb{P}^{N} \times X$. В следуюшем разделе доказывается, что функтор $F$ изоморфен функтору $\Phi_{\mathscr{E}}$.

Выберем очень обильное линейное расслоение $\mathscr{L}$ на многообразии $M$ такое, что $\mathrm{H}^{i}\left(\mathscr{L}^{k}\right)=0$ для всех $k>0$ и всех $i \neq 0$. Через $j$ обозначим замкнутое вложение многообразия $M$ в проективное пространство $\mathbb{P}^{N}$, задаваемое расслоением $\mathscr{L}$. 
На произведении $\mathbb{P}^{N} \times \mathbb{P}^{N}$ существует так называемая резольвента диагонали (см. [2]). Это комплекс пучков следующего вида:

$$
\begin{aligned}
0 \longrightarrow \mathscr{O}(-N) \otimes \Omega^{N}(N) & \stackrel{d_{-N}}{\longrightarrow} \mathscr{O}(-N+1) \otimes \Omega^{N-1}(N-1) \\
& \longrightarrow \cdots \longrightarrow \mathscr{O}(-1) \otimes \Omega^{1}(1) \stackrel{d_{-1}}{\longrightarrow} \mathscr{O} \otimes \mathscr{O} .
\end{aligned}
$$

Этот комплекс является резольвентой структурного пучка $\mathscr{O}_{\Delta}$, где $\Delta-$ диагональ на произведении $\mathbb{P}^{N} \times \mathbb{P}^{N}$.

Обозначим через $F^{\prime}$ функтор из категории $\mathbf{D}^{b}\left(\mathbb{P}^{N}\right)$ в категорию $\mathbf{D}^{b}(X)$, которьй является композищией $F \circ \mathbf{L} j^{*}$. Рассмотрим диаграмму проекций

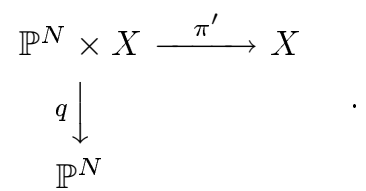

Обозначим через

$$
d_{-i}^{\prime} \in \operatorname{Hom}_{\mathbb{P}^{N} \times X}\left(\mathscr{O}(-i) \otimes F^{\prime}\left(\Omega^{i}(i)\right), \mathscr{O}(-i+1) \otimes F^{\prime}\left(\Omega^{i-1}(i-1)\right)\right)
$$

образ морфизма $d_{-i}$ относительно следуюшего сквозного отображения:

$$
\begin{aligned}
\operatorname{Hom} & \left(\mathscr{O}(-i) \otimes \Omega^{i}(i), \mathscr{O}(-i+1) \otimes \Omega^{i-1}(i-1)\right) \\
& \stackrel{\sim}{\longrightarrow} \operatorname{Hom}\left(\mathscr{O} \otimes \Omega^{i}(i), \mathscr{O}(1) \otimes \Omega^{i-1}(i-1)\right) \\
& \stackrel{\sim}{\longrightarrow} \operatorname{Hom}\left(\Omega^{i}(i), H^{0}(\mathscr{O}(1)) \otimes \Omega^{i-1}(i-1)\right) \\
& \longrightarrow \operatorname{Hom}\left(F^{\prime}\left(\Omega^{i}(i)\right), H^{0}(\mathscr{O}(1)) \otimes F^{\prime}\left(\Omega^{i-1}(i-1)\right)\right) \\
& \stackrel{\sim}{\longrightarrow} \operatorname{Hom}\left(\mathscr{O} \otimes F^{\prime}\left(\Omega^{i}(i)\right), \mathscr{O}(1) \otimes F^{\prime}\left(\Omega^{i-1}(i-1)\right)\right) \\
& \stackrel{\sim}{\longrightarrow} \operatorname{Hom}\left(\mathscr{O}(-i) \otimes F^{\prime}\left(\Omega^{i}(i)\right), \mathscr{O}(-i+1) \otimes F^{\prime}\left(\Omega^{i-1}(i-1)\right)\right) .
\end{aligned}
$$

Несложно проверить, что композиция $d_{-i+1}^{\prime} \circ d_{-i}^{\prime}$ равна 0. Следовательно, можно рассмотреть следующий ограниченньй комплекс объектов производной категории $\mathbf{D}^{b}\left(\mathbb{P}^{N} \times X\right):$

$$
C^{\cdot}:=\left\{\mathscr{O}(-N) \otimes F^{\prime}\left(\Omega^{N}(N)\right) \stackrel{d_{-N}^{\prime}}{\longrightarrow} \cdots \longrightarrow \mathscr{O}(-1) \otimes F^{\prime}\left(\Omega^{1}(1)\right) \stackrel{d_{-1}^{\prime}}{\longrightarrow} \mathscr{O} \otimes F^{\prime}(\mathscr{O})\right\} .
$$

При $l<0$ имеем

$$
\begin{aligned}
& \operatorname{Hom}^{l}\left(\mathscr{O}(-i) \otimes F^{\prime}\left(\Omega^{i}(i)\right), \mathscr{O}(-k) \otimes F^{\prime}\left(\Omega^{k}(k)\right)\right) \\
& \cong \operatorname{Hom}^{l}\left(\mathscr{O} \otimes F^{\prime}\left(\Omega^{i}(i)\right), \mathrm{H}^{0}(\mathscr{O}(i-k)) \otimes F^{\prime}\left(\Omega^{k}(k)\right)\right) \\
& \cong \operatorname{Hom}^{l}\left(j^{*}\left(\Omega^{i}(i)\right), \mathrm{H}^{0}(\mathscr{O}(i-k)) \otimes j^{*}\left(\Omega^{k}(k)\right)\right)=0 .
\end{aligned}
$$

Следовательно, по лемме 3.1.2 существует свертка для комплекса $C \cdot$, и все свертки изоморфны. Обозначим через $\mathscr{E}^{\prime}$ свертку комплекса $C^{\cdot}$, а через $\gamma_{0}$ обозначим морфизм $\mathscr{O} \otimes F^{\prime}(\mathscr{O}) \stackrel{\gamma_{0}}{\longrightarrow} \mathscr{E}^{\prime}$. (На самом деле далее будет видно, что все свертки комплекса $C^{\cdot}$ изоморфны друг другу канонически.) Пусть теперь $\Phi_{\mathscr{E}},-$ функтор из категории $\mathbf{D}^{b}\left(\mathbb{P}^{N}\right)$ в категорию $\mathbf{D}^{b}(X)$, определенньй формулой $(7)$. 
Лемма 3.3.1. Для всех $k \in \mathbb{Z}$ существуют канонические изоморфизмы

$$
f_{k}: F^{\prime}(\mathscr{O}(k)) \stackrel{\sim}{\longrightarrow} \Phi_{\mathscr{E}}(\mathscr{O}(k)),
$$

и эти изоморфизмы функториальны, т.е. для любого $\alpha: \mathscr{O}(k) \rightarrow \mathscr{O}(l)$ диаграмма

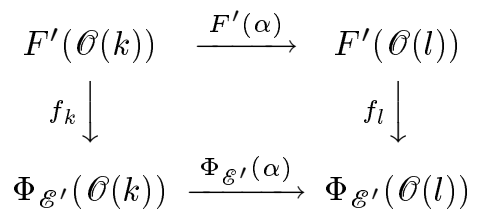

является коммутативной.

ДокАЗАТЕльство. Сначала предположим, что $k \geqslant 0$.

Рассмотрим резольвенту (31) для диагонали $\Delta \subset \mathbb{P}^{N} \times \mathbb{P}^{N}$. Умножим ее тензорно на $\mathscr{O}(k) \otimes \mathscr{O}$ и возьмем прямой образ относительно проекции на второй сомножитель. В результате получим следующую резольвенту пучка $\mathscr{O}(k)$ на проективном пространстве $\mathbb{P}^{N}$ :

$\left\{\mathrm{H}^{0}(\mathscr{O}(k-N)) \otimes \Omega^{N}(N) \longrightarrow \cdots \longrightarrow \mathrm{H}^{0}(\mathscr{O}(k-1)) \otimes \Omega^{1}(1) \longrightarrow \mathrm{H}^{0}(\mathscr{O}(k)) \otimes \mathscr{O}\right\} \stackrel{\delta_{k}}{\longrightarrow} \mathscr{O}(k)$.

Из условия точности функтора $F^{\prime}$ следует, что объект $F^{\prime}(\mathscr{O}(k))$ является сверткой комплекса

$\mathrm{H}^{0}(\mathscr{O}(k-N)) \otimes F^{\prime}\left(\Omega^{N}(N)\right) \longrightarrow \cdots \longrightarrow \mathrm{H}^{0}(\mathscr{O}(k-1)) \otimes F^{\prime}\left(\Omega^{1}(1)\right) \longrightarrow \mathrm{H}^{0}(\mathscr{O}(k)) \otimes F^{\prime}(\mathscr{O})$

объектов категории $\mathbf{D}^{b}(X)$. Обозначим этот комплекс через $D_{k}^{*}$.

Теперь вспомним, что по построению объект $\mathscr{E}^{\prime}$ является сверткой комплекса $C$. (32). Рассмотрим комплекс $C_{k}^{\cdot}:=q^{*} \mathscr{O}(k) \otimes C^{\cdot}$ на $\mathbb{P}^{N} \times X$. Объект $q^{*} \mathscr{O}(k) \otimes \mathscr{E}^{\prime}$ является его сверткой. И имеется морфизм $\gamma_{k}: \mathscr{O}(k) \otimes F^{\prime}(\mathscr{O}) \longrightarrow q^{*} \mathscr{O}(k) \otimes \mathscr{E}^{\prime}$, канонически получаюшийся из $\gamma_{0}$. Комплекс $\pi_{*}^{\prime}\left(C_{\dot{k}}\right)$, которьй есть прямой образ комплекса $\left(C_{k}\right)$ при проекции на второй сомножитель, канонически изоморфен комплексу $D_{k}^{*}$. Таким образом, видим, что объекты $F^{\prime}(\mathscr{O}(k))$ и $\Phi_{\mathscr{E}}(\mathscr{O}(k)):=\mathbf{R} \pi_{*}^{\prime}\left(q^{*} \mathscr{O}(k) \otimes \mathscr{E}^{\prime}\right)$ оба являются свертками одного и того же комплекса $D_{k}$.

По предположению функтор $F$ является полным и строгим. Следовательно, для локально свободных пучков $\mathscr{G}$ и $\mathscr{H}$ на $\mathbb{P}^{N}$ при $i<0$ имеем равенство

$$
\operatorname{Hom}^{i}\left(F^{\prime}(\mathscr{G}), F^{\prime}(\mathscr{H})\right)=\operatorname{Hom}^{i}\left(j^{*}(\mathscr{G}), j^{*}(\mathscr{H})\right)=0 .
$$

Это, в частности, влечет, что комплекс $D_{k}^{*}$ удовлетворяет условиям (25) и $(26)$ леммы 3.1.2. Следовательно, по этой лемме существует однозначно определенньй изоморфизм $f_{k}: F^{\prime}(\mathscr{O}(k)) \stackrel{\sim}{\longrightarrow} \Phi_{\mathscr{E}}(\mathscr{O}(k))$, делающий коммутативной следующую диаграмму:

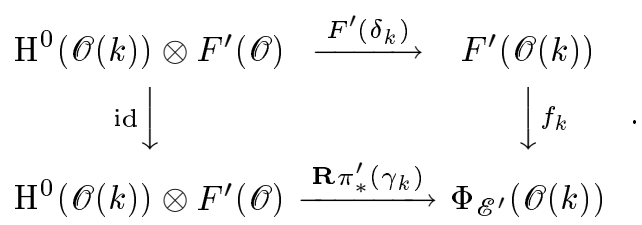


Теперь покажем, что эти изоморфизмы функториальны. Для любого $\alpha: \mathscr{O}(k) \rightarrow$ $\mathscr{O}(l)$ имеются коммутативные квадраты:

и

$$
\begin{array}{ll}
\mathrm{H}^{0}(\mathscr{O}(k)) \otimes F^{\prime}(\mathscr{O}) \stackrel{F^{\prime}\left(\delta_{k}\right)}{\longrightarrow} F^{\prime}(\mathscr{O}(k)) \\
\mathrm{H}^{0}(\alpha) \otimes \mathrm{id} \downarrow & \downarrow F^{\prime}(\alpha) \\
\mathrm{H}^{0}(\mathscr{O}(l)) \otimes F^{\prime}(\mathscr{O}) \stackrel{F^{\prime}\left(\delta_{l}\right)}{\longrightarrow} F^{\prime}(\mathscr{O}(l))
\end{array}
$$

$$
\begin{array}{ll}
\mathrm{H}^{0}(\mathscr{O}(k)) \otimes F^{\prime}(\mathscr{O}) \stackrel{\mathbf{R} \pi_{*}^{\prime}\left(\gamma_{k}\right)}{\longrightarrow} & \Phi_{\mathscr{E}^{\prime}}(\mathscr{O}(k)) \\
\mathrm{H}^{0}(\alpha) \otimes \mathrm{id} \downarrow & \downarrow \Phi_{\mathscr{E}^{\prime}}(\alpha) . \\
\mathrm{H}^{0}(\mathscr{O}(l)) \otimes F^{\prime}(\mathscr{O}) \stackrel{\mathbf{R} \pi_{*}^{\prime}\left(\gamma_{l}\right)}{\longrightarrow} \Phi_{\mathscr{E}^{\prime}}(\mathscr{O}(l))
\end{array}
$$

Из этих трех коммутативных квадратов получаются следующие равенства:

$$
\begin{gathered}
f_{l} \circ F^{\prime}(\alpha) \circ F^{\prime}\left(\delta_{k}\right)=f_{l} \circ F^{\prime}\left(\delta_{l}\right) \circ\left(\mathrm{H}^{0}(\alpha) \otimes \mathrm{id}\right)=\mathbf{R} \pi_{*}^{\prime}\left(\gamma_{l}\right) \circ\left(\mathrm{H}^{0}(\alpha) \otimes \mathrm{id}\right), \\
\Phi_{\mathscr{E}^{\prime}}(\alpha) \circ f_{k} \circ F^{\prime}\left(\delta_{k}\right)=\Phi_{\mathscr{E}}(\alpha) \circ \mathbf{R} \pi_{*}^{\prime}\left(\gamma_{k}\right)=\mathbf{R} \pi_{*}^{\prime}\left(\gamma_{l}\right) \circ\left(\mathrm{H}^{0}(\alpha) \otimes \mathrm{id}\right) .
\end{gathered}
$$

Комплексы $D_{k}^{*}$ и $D_{i}$ удовлетворяют условиям леммы 3.1.3, и, следовательно, сушествует единственньй морфизм $h: F^{\prime}(\mathscr{O}(k)) \rightarrow \Phi_{\mathscr{E}}(\mathscr{O}(l))$, для которого

$$
h \circ F^{\prime}\left(\delta_{k}\right)=\mathbf{R} \pi_{*}^{\prime}\left(\gamma_{l}\right) \circ\left(\mathrm{H}^{0}(\alpha) \otimes \mathrm{id}\right) .
$$

Таким образом, морфизм $h$ совпадает одновременно с $f_{l} \circ F^{\prime}(\alpha)$ и $\Phi_{\mathscr{E}}(\alpha) \circ f_{k}$, что влечет равенство двух последних.

Теперь рассмотрим случай $k<0$.

Возьмем правую резольвенту

$$
\mathscr{O}(k) \stackrel{\sim}{\longrightarrow}\left\{V_{0}^{k} \otimes \mathscr{O} \longrightarrow \cdots \longrightarrow V_{N}^{k} \otimes \mathscr{O}(N)\right\}
$$

для пучка $\mathscr{O}(k)$ на $\mathbb{P}^{N}$. Опять применяя лемму 3.1 .3$, находим, что морфизм комплексов

$$
\begin{aligned}
& V_{0}^{k} \otimes F^{\prime}(\mathscr{O}) \longrightarrow \cdots \longrightarrow V_{N}^{k} \otimes F^{\prime}(\mathscr{O}(N)) \\
& \operatorname{id} \otimes f_{0} \downarrow^{2} \quad \operatorname{id} \otimes f_{N} \downarrow^{2} \\
& V_{0}^{k} \otimes \Phi_{\mathscr{E}^{\prime}}(\mathscr{O}) \longrightarrow \cdots \longrightarrow V_{N}^{k} \otimes \Phi_{\mathscr{E}^{\prime}}(\mathscr{O}(N))
\end{aligned}
$$

задает однозначно определенньй морфизм $f_{k}: F^{\prime}(\mathscr{O}(k)) \longrightarrow \Phi_{\mathscr{E}}(\mathscr{O}(k))$. Непосредственная проверка, которую мы опускаем, дает нам функториальность этих морфизмов.

ЗАмечАнИЕ 3.3.2. Заметим, что объект $\mathscr{E}^{\prime} \in \mathbf{D}^{b}\left(\mathbb{P}^{N} \times X\right)$, построенньй по функтору $F$, определен однозначно.

Теперь докажем существование такого объекта в категории $\mathscr{E} \in \mathbf{D}^{b}(M \times X)$, что $\mathbf{R} J_{*} \mathscr{E} \cong \mathscr{E}^{\prime}$, где, как и выше, $J$ - вложение $M \times X$ в $\mathbb{P}^{N} \times X$.

Пусть $\mathscr{L}$ - очень обильное линейное расслоение на $M$ и $j: M \hookrightarrow \mathbb{P}^{N}$ - вложение в проективное пространство, задаваемое этим расслоением $\mathscr{L}$. Обозначим через $A$ градуированную алгебру $\bigoplus_{i=0}^{\infty} \mathrm{H}^{0}\left(M, \mathscr{L}^{i}\right)$.

Положим $B_{0}=k$ и $B_{1}=A_{1}$. Для $m \geqslant 2$ определим $B_{m}$ по правилу

$$
B_{m}=\operatorname{Ker}\left(B_{m-1} \otimes A_{1} \stackrel{u_{m-1}}{\longrightarrow} B_{m-2} \otimes A_{2}\right),
$$

где $u_{m-1}-$ естественное отображение, определяемое по индукции. 
ОПРЕДЕЛЕНИЕ 3.3.3. Назовем алгебру $A$ n-кошулевой, если следующая последовательность правых $A$-модулей

$$
B_{n} \otimes_{k} A \longrightarrow B_{n-1} \otimes_{k} A \longrightarrow \cdots \longrightarrow B_{1} \otimes_{k} A \longrightarrow A \longrightarrow k \longrightarrow 0
$$

точна. Алгебра назьвается кошулевой, если она $n$-кошулева для любого $n$.

Предположим, что алгебра $A$ является $n$-кошулевой. Положим $R_{0}=\mathscr{O}_{M}$, а для $m \geqslant 1$ обозначим через $R_{m}$ ядро канонического морфизма

$$
B_{m} \otimes \mathscr{O}_{M} \longrightarrow B_{m-1} \otimes \mathscr{L},
$$

определенного естественным вложением $B_{m} \longrightarrow B_{m-1} \otimes A_{1}$. Используя (33), получаем канонический морфизм $R_{m} \longrightarrow A_{1} \otimes R_{m-1}$ (на самом деле можно проверить, что $\left.\operatorname{Hom}\left(R_{m}, R_{m-1}\right) \cong A_{1}^{*}\right)$.

Более того, при $m \leqslant n \quad n$-кошулевость алгебры $A$ влечет точность следуюшего комплекса пучков:

$$
0 \longrightarrow R_{m} \longrightarrow B_{m} \otimes \mathscr{O}_{M} \longrightarrow B_{m-1} \otimes \mathscr{L} \longrightarrow \cdots \longrightarrow B_{1} \otimes \mathscr{L}^{m-1} \longrightarrow \mathscr{L}^{m} \longrightarrow 0 \text {. }
$$

На проективном пространстве $\mathbb{P}^{N}$ существует точный комплекс вида

$$
0 \longrightarrow \Omega^{m}(m) \longrightarrow \Lambda^{m} A_{1} \otimes \mathscr{O} \longrightarrow \Lambda^{m-1} A_{1} \otimes \mathscr{O}(1) \longrightarrow \cdots \longrightarrow \mathscr{O}(m) \longrightarrow 0 .
$$

Существует каноническое отображение $f_{m}: j^{*} \Omega^{m}(m) \longrightarrow R_{m}$. Действительно, так как алгебра $A$ коммутативна, то имеются естественные вложения $\Lambda^{i} A_{1} \subset B_{i}$. Поэтому существует морфизм из комплекса (35), ограниченного на $M$, в комплекс (34) и, значит, каноническое отображение $f_{m}: j^{*} \Omega^{m}(m) \longrightarrow R_{m}$.

Известно, что для любого $n$ сушествует такое $l$, что алгебра Веронезе $A^{l}=$ $\bigoplus_{i=0}^{\infty} \mathrm{H}^{0}\left(M, \mathscr{L}^{i l}\right)$ является $n$-кошулевой (более того, в статье [1] доказано, что на самом деле $A^{l}$ является кошулевой для $\left.l \gg 0\right)$.

В дальнейшем, однако, кроме $n$-кошулевости алгебры Веронезе нам будут нужны некоторые дополнительные свойства. А именно, используя технику статьи [18] и заменяя пучок $\mathscr{L}$ на достаточно большую степень $\mathscr{L}^{j}$, можно доказать следующее предложение.

ПРЕДЛОЖЕНИЕ 3.3.4. Для всякого челого $n$ можсно найти очень обильное линейное расслоение $\mathscr{L}$ такое, что

1) алгебра $A$ является $n$-кошулевой, т.е. последовательность

$$
B_{n} \otimes_{k} A \longrightarrow B_{n-1} \otimes_{k} A \longrightarrow \cdots \longrightarrow B_{1} \otimes_{k} A \longrightarrow A \longrightarrow k \longrightarrow 0
$$

точна;

2) комплекс пучков на $M$

$A_{k-n} \otimes R_{n} \longrightarrow A_{k-n+1} \otimes R_{n-1} \longrightarrow \cdots \longrightarrow A_{k-1} \otimes R_{1} \longrightarrow A_{k} \otimes R_{0} \longrightarrow \mathscr{L}^{k} \longrightarrow 0$

точен для всех $k \geqslant 0\left(\right.$ если $k-i<0$, тогда по определению $\left.A_{k-i}=0\right)$;

3) комплекс пучков на $M \times M$

$$
\mathscr{L}^{-n} \otimes R_{n} \longrightarrow \cdots \longrightarrow \mathscr{L}^{-1} \otimes R_{1} \longrightarrow \mathscr{O}_{M} \otimes R_{0} \longrightarrow \mathscr{O}_{\Delta}
$$

является точным, т.е. дает п-резольвенту диагонали на $M \times M$. 
Доказательство этого предложения дается в разделе 3.5.

Обозначим через $T_{k}$ ядро канонического морфизма $A_{k-n} \otimes R_{n} \longrightarrow A_{k-n+1} \otimes R_{n-1}$. Учитьвая свойство 2) предложения 3.3.4 и то, что $\operatorname{Ext}^{n+1}\left(\mathscr{L}^{k}, T_{k}\right)=0$ для $n \gg 0$, находим, что любая свертка комплекса

$$
A_{k-n} \otimes R_{n} \longrightarrow A_{k-n+1} \otimes R_{n-1} \longrightarrow \cdots \longrightarrow A_{k} \otimes R_{0}
$$

канонически изоморфна $T_{k}[n] \oplus \mathscr{L}^{k}$.

Канонические морфизмы $R_{k} \longrightarrow A_{1} \otimes R_{k-1}$ индуцируют морфизмы $\mathscr{L}^{-k} \otimes F\left(R_{k}\right)$ $\longrightarrow \mathscr{L}^{-k+1} \otimes F\left(R_{k-1}\right)$. Это следует из сушествования изоморфизмов:

$$
\begin{aligned}
\operatorname{Hom}\left(\mathscr{L}^{-k} \otimes F\left(R_{k}\right), \mathscr{L}^{-k+1} \otimes F\left(R_{k-1}\right)\right) & \cong \operatorname{Hom}\left(F\left(R_{k}\right), \mathrm{H}^{0}(\mathscr{L}) \otimes F\left(R_{k-1}\right)\right) \\
& \cong \operatorname{Hom}\left(R_{k}, A_{1} \otimes R_{k-1}\right) .
\end{aligned}
$$

Более того, имеется следующий комплекс объектов в категории $\mathbf{D}^{b}(M \times X)$ :

$$
\mathscr{L}^{-n} \otimes F\left(R_{n}\right) \longrightarrow \cdots \longrightarrow \mathscr{L}^{-1} \otimes F\left(R_{1}\right) \longrightarrow \mathscr{O}_{M} \otimes F\left(R_{0}\right) .
$$

По лемме 3.1.2 комплекс (36) обладает сверткой и все свертки изоморфны. Обозначим эту свертку через $G \in \mathbf{D}^{b}(M \times X)$.

Для любого $k \geqslant 0$ объект $\mathbf{R} \pi_{*}\left(G \otimes p^{*}\left(\mathscr{L}^{k}\right)\right)$ является сверткой комплекса

$$
A_{k-n} \otimes F\left(R_{n}\right) \longrightarrow A_{k-n+1} \otimes F\left(R_{n-1}\right) \longrightarrow \cdots \longrightarrow A_{k} \otimes F\left(R_{0}\right) .
$$

С другой стороны, объект $F\left(T_{k}[n] \oplus \mathscr{L}^{k}\right)$ также является сверткой этого комплекса, которьй очевидно удовлетворяет условию леммы 3.1.2. Следовательно, имеется изоморфизм $\mathbf{R} \pi_{*}\left(G \otimes p^{*}\left(\mathscr{L}^{k}\right)\right) \cong F\left(T_{k}[n] \oplus \mathscr{L}^{k}\right)$.

Из леммы 3.2.3 и замечания 3.2.4 следует, что для всех $k>0$ нетривиальные пучки когомологий $H^{i}\left(\mathbf{R} \pi_{*}\left(G \otimes p^{*}\left(\mathscr{L}^{k}\right)\right)\right)=H^{i}\left(F\left(T_{k}\right)[n]\right) \oplus H^{i}\left(F\left(\mathscr{L}^{k}\right)\right)$ сконцентрированы на объединении $[-n-a,-n] \cup[-a, 0]$ (число $a$ определено в замечании 3.2.4). Так как пучок $\mathscr{L}$ обилен, то получаем, что пучки когомологий $H^{i}(G)$ также сконцентрированы на $[-n-a,-n] \cup[-a, 0]$. Можно предполагать, что $n>\operatorname{dim} M+\operatorname{dim} X+a$. В этом случае, так как категория когерентных пучков на многообразии $M \times X$ имеет гомологическую размерность, равную $\operatorname{dim} M+\operatorname{dim} X$, получаем, что $G \cong C \oplus \mathscr{E}$, где $\mathscr{E}, C$ - такие объекты категории $\mathbf{D}^{b}(M \times X)$, для которых $H^{i}(\mathscr{E})=0$ при $i \notin[-a, 0]$ и $H^{i}(C)=0$ при $i \notin[-n-a,-n]$. Отсюда, в частности, получаем, что $\mathbf{R} \pi_{*}\left(\mathscr{E} \otimes p^{*}\left(\mathscr{L}^{k}\right)\right) \cong F\left(\mathscr{L}^{k}\right)$. Заметим, что так как объект $G$ определен однозначно как свертка комплекса (36), то и объект $\mathscr{E}$ также определен однозначно с точностью до изоморфизма.

Теперь покажем, что существует изоморфизм $\mathbf{R} J_{*} \mathscr{E} \cong \mathscr{E}^{\prime}$. Для этого рассмотрим отображение комплексов над $\mathbf{D}^{b}\left(\mathbb{P}^{N} \times X\right)$

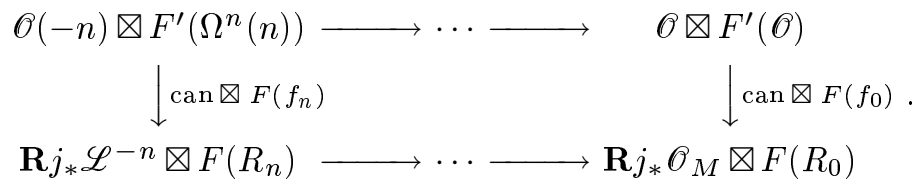

Применяя лемму 3.1 .3 , получаем сушествование морфизма между свертками $\varphi: K \longrightarrow$ $\mathbf{R} J_{*} G$. 
Если $N>n$, тогда объект $K$ неизоморфен $\mathscr{E}^{\prime}$, но существует выделенный треугольник

$$
S \longrightarrow K \longrightarrow \mathscr{E}^{\prime} \longrightarrow S[1]
$$

Таким же образом, как и вьше, проверяется, что пучки когомологий $H^{i}(S) \neq 0$, только если $i \in[-n-a,-n]$. Это влечет, что $\operatorname{Hom}\left(S, \mathbf{R} J_{*} \mathscr{E}\right)=0$ и $\operatorname{Hom}\left(S[1], \mathbf{R} J_{*} \mathscr{E}\right)=0$, так как когомологии $\mathbf{R} J_{*} \mathscr{E}$ сконцентрированы на отрезке [-a,0]. Отсюда следует существование единственного морфизма $\psi: \mathscr{E}^{\prime} \longrightarrow \mathbf{R} J_{*} \mathscr{E}$, делающего коммутативной диаграмму

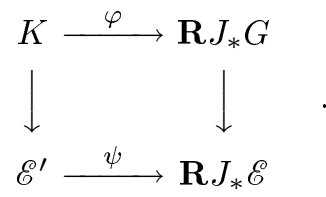

Нам известно, что

$$
\mathbf{R} \pi_{*}^{\prime}\left(\mathscr{E}^{\prime} \otimes q^{*}(\mathscr{O}(k))\right) \cong F\left(\mathscr{L}^{k}\right) \cong \mathbf{R} \pi_{*}\left(\mathscr{E} \otimes p^{*}\left(\mathscr{L}^{k}\right)\right)
$$

Обозначим через $\psi_{k}$ морфизмы $\mathbf{R} \pi_{*}^{\prime}\left(\mathscr{E}^{\prime} \otimes q^{*}(\mathscr{O}(k))\right) \longrightarrow \mathbf{R} \pi_{*}\left(\mathscr{E} \otimes p^{*}\left(\mathscr{L}^{k}\right)\right)$, индуцированные морфизмом $\psi$. Морфизмы $\psi_{k}$ могут быть включены в коммутативную диаграмму:

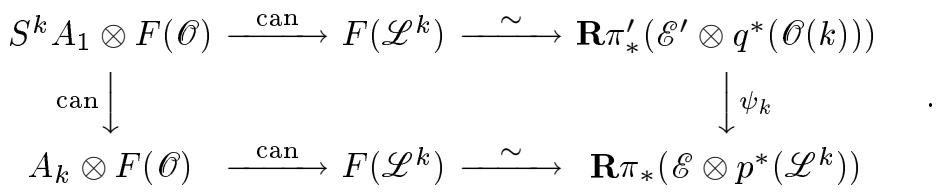

Из этого получаем, что $\psi_{k}$ являются изоморфизмами для всех $k \geqslant 0$. Следовательно, $\psi$ также есть изоморфизм. Таким образом, получено доказательство следуюшего утверждения.

ПРЕДЛОЖЕНИЕ 3.3.5. Существует единственный с точностью до изоморфизма оббект $\mathscr{E} \in \mathbf{D}^{b}(M \times X)$ такой, ито $\mathbf{R} J_{*} \mathscr{E} \cong \mathscr{E}^{\prime}$, әде $\mathscr{E}^{\prime}-$ оббект из $\mathbf{D}^{b}\left(\mathbb{P}^{N} \times X\right)$, построеный в разделе 3.3 .

3.4. Доказательство основной теоремы. В предыдущем разделе, стартуя с вполне строгого функтора $F$ между производными категориями когерентных пучков на многообразиях $M$ и $X$, был построен объект $\mathscr{E}$ на произведении этих многообразий и, тем самым, получен новый функтор $\Phi \mathscr{E}$. Основная цель данного раздела - показать, что эти два функтора $F$ и $\Phi_{\mathscr{E}}$ изоморфны. Для этого нам нужно построить естественное преобразование между данными функторами, которое и будет осуществлять изоморфизм. По построению, преобразование уже задано на обильной последовательности линейных расслоений на $M$. Наша задача - продолжить это преобразование на всю производную категорию.

Вначале дадим доказательства некоторых утверждений про абелевы категории, которые понадобятся нам в дальнейшем.

Пусть $\mathscr{A}-k$-линейная абелева категория (далее всегда рассматриваются абелевы категории, которые $k$-линейны). 
ОПРЕДЕЛЕНИЕ 3.4.1. Пусть $\left\{P_{i} \mid i \in \mathbb{Z}_{\leqslant 0}\right\}$ - последовательность объектов из абелевой категории $\mathscr{A}$. Будем назьвать такую последовательность обильной, если для каждого объекта $X \in \mathscr{A}$ существует целое число $N$ такое, что для всех $i<N$ вьполнены следуюшие условия:

a) канонический морфизм $\operatorname{Hom}\left(P_{i}, X\right) \otimes P_{i} \longrightarrow X$ является сюръективным,

b) $\operatorname{Ext}^{j}\left(P_{i}, X\right)=0$ для всех $j \neq 0$,

c) $\operatorname{Hom}\left(X, P_{i}\right)=0$.

ПРИмЕР 3.4.2. Если $\mathscr{L}$ - обильное линейное расслоение на проективном многообразии, тогда последовательность $\left\{\mathscr{L}^{i} \mid i \in \mathbb{Z}_{\leqslant 0}\right\}$ является обильной последовательностью в абелевой категории когерентных пучков.

ЛЕмма 3.4.3. Пусть $\left\{P_{i}\right\}$ - обильная последовательность в абелевой категории $\mathscr{A}$. Тогда, если для обгекта $X$ из категории $\mathbf{D}^{b}(\mathscr{A})$ выполняется равенство

$$
\operatorname{Hom}^{\cdot}\left(P_{i}, X\right)=0
$$

для всех $i \ll 0$, то $X$ является нулевым обгектом.

ДоКАЗАТЕЛЬСТВО. Из определения обильности для $i \ll 0$ получаем

$$
\operatorname{Hom}\left(P_{i}, H^{k}(X)\right) \cong \operatorname{Hom}^{k}\left(P_{i}, X\right)=0 .
$$

Но морфизм $\operatorname{Hom}\left(P_{i}, H^{k}(X)\right) \otimes P_{i} \longrightarrow H^{k}(X)$ должен быть сюръективен при $i \ll 0$. Следовательно, $H^{k}(X)=0$ для всех $k$. То есть $X$-нулевой объект.

Лемма 3.4.4. Пусть $\mathscr{A}$ - абелева категория конечной гомологической размерности, а $\left\{P_{i}\right\}$ - обильная последовательность в абелевой категории $\mathcal{A}$. Тогда, если обгект $X \in \mathbf{D}^{b}(\mathscr{A})$ такой, что $\operatorname{Hom}^{\cdot}\left(X, P_{i}\right)=0$ для всех $i \ll 0$, то $X$ является нулевым облектом.

ДокАЗАТЕльство. Предположим, что объект $X$ нетривиален. Сдвигая этот объект в производной категории, если это необходимо, можно предполагать, что крайняя правая ненулевая когомология объекта $X$ имеет номер 0. Рассмотрим канонический морфизм $X \longrightarrow H^{0}(X)$. Найдется номер $i_{1}$, для которого сушествует сюръективное отображение $P_{i_{1}}^{\oplus k_{1}} \longrightarrow H^{0}(X)$. Обозначим через $Y_{1}$ его ядро. По предположению $\operatorname{Hom}^{\cdot}\left(X, P_{i_{1}}\right)=0$, и, значит, $\operatorname{Hom}^{1}\left(X, Y_{1}\right) \neq 0$. Далее возьмем сюръективное отображение $P_{i_{2}}^{\oplus k_{2}} \longrightarrow Y_{1}$, которое существует для достаточно отрищательного номера $i_{2}$. Через $Y_{2}$ обозначим ядро этого отображения. Снова условие $\operatorname{Hom}\left(X, P_{i_{2}}\right)=0$ дает нам $\operatorname{Hom}^{2}\left(X, Y_{2}\right) \neq 0$. Продолжая эту процедуру, получим противоречие с конечностью гомологической размерности категории $\mathscr{A}$.

Лемма 3.4.5. Пусть $\mathscr{A}$ и $\mathscr{B}$ - абелевь категории и категория $\mathscr{A}$ имеет конечную гомологическую размерность. Пусть $\left\{P_{i}\right\}$ - обильная последовательность в категории $\mathscr{A}$. Предположим, что $F-$ точный функтор из категории $\mathbf{D}^{b}(\mathscr{A})$ в категорию $\mathbf{D}^{b}(\mathscr{B})$, который имеет правый и левый сопряженные функторы $F^{!}$ и $F^{*}$ соответственно. Если отображения

$$
\operatorname{Hom}^{k}\left(P_{j}, P_{i}\right) \stackrel{\sim}{\longrightarrow} \operatorname{Hom}^{k}\left(F\left(P_{j}\right), F\left(P_{i}\right)\right)
$$

суть изоморфизмы при $j<i$ и для всех $k$, тогда функтор $F$ является вполне строгим. 
ДокАЗАТЕЛЬСТво. Возьмем канонический морфизм $f_{i}: P_{i} \longrightarrow F^{!} F\left(P_{i}\right)$ и рассмотрим выделенньй треугольник

$$
P_{i} \stackrel{f_{i}}{\longrightarrow} F^{!} F\left(P_{i}\right) \longrightarrow C_{i} \longrightarrow P_{i}[1] .
$$

По предположению при $j<i$ имеем изоморфизмы:

$$
\operatorname{Hom}^{k}\left(P_{j}, P_{i}\right) \stackrel{\sim}{\longrightarrow} \operatorname{Hom}^{k}\left(F\left(P_{j}\right), F\left(P_{i}\right)\right) \cong \operatorname{Hom}^{k}\left(P_{j}, F^{!} F\left(P_{i}\right)\right) .
$$

Таким образом, $\operatorname{Hom}^{\cdot}\left(P_{j}, C_{i}\right)=0$ при $j<i$. Из леммы 3.4 .3 следует, что $C_{i}=0$. И, значит, $f_{i}$ является изоморфизмом.

Теперь для произвольного объекта $X$ рассмотрим каноническое отображение $g_{X}: F^{*} F(X) \longrightarrow X$ и выделенньй треугольник

$$
F^{*} F(X) \stackrel{g_{X}}{\longrightarrow} X \longrightarrow C_{X} \longrightarrow F^{*} F(X)[1] .
$$

Имеется последовательность изоморфизмов

$$
\operatorname{Hom}^{k}\left(X, P_{i}\right) \stackrel{\sim}{\longrightarrow} \operatorname{Hom}^{k}\left(X, F^{!} F\left(P_{i}\right)\right) \cong \operatorname{Hom}^{k}\left(F^{*} F(X), P_{i}\right) .
$$

Отсюда следует, что $\operatorname{Hom}^{*}\left(C_{X}, P_{i}\right)=0$ для всех $i$. По лемме 3.4 .4 получаем, что $C_{X}=$ 0 . И, значит, $g_{X}-$ изоморфизм. Что и доказьвает, что функтор $F$ является вполне строгим.

Теперь сформулируем и докажем основное предложение этого раздела, которое необходимо нам для доказательства основной теоремы 3.2.1 этой главы. Надо отметить, что данное предложение представляет также самостоятельный интерес.

Пусть $\mathscr{A}$ - абелева категория с обильной последовательностью $\left\{P_{i} \mid i \in \mathbb{Z}_{\leqslant 0}\right\}$. Обозначим через $j$ вложение полной подкатегории $\mathscr{C}$ с объектами $\mathrm{Ob} \mathscr{C}:=\left\{P_{i} \mid i \in\right.$ $\left.\mathbb{Z}_{\leqslant 0}\right\}$ в категорию $\mathbf{D}^{b}(\mathscr{A})$. В этой ситуации можно показать, что если для функтора $F: \mathbf{D}^{b}(\mathscr{A}) \longrightarrow \mathbf{D}^{b}(\mathscr{A})$ сушествует изоморфизм $\left.F\right|_{\mathscr{C}}$ с тождественным функтором на $\mathscr{C}$, тогда это преобразование может быть продолжено до изоморфизма на всю $\mathbf{D}^{b}(\mathscr{A})$.

ПРЕДЛОЖЕНИЕ 3.4.6. Пусть $\mathscr{A}$ - абелева категория с обильной последовательностью $\left\{P_{i} \mid i \in \mathbb{Z}_{\leqslant 0}\right\}$. Обозначим через $j$ вложсение полной подкатегории $\mathscr{C}$ с обгектами $\mathrm{Ob} \mathscr{C}:=\left\{P_{i} \mid i \in \mathbb{Z}_{\leqslant 0}\right\}$ в категорию $\mathbf{D}^{b}(\mathscr{A})$. Пусть $F: \mathbf{D}^{b}(\mathscr{A}) \longrightarrow \mathbf{D}^{b}(\mathscr{A})$ - некоторая автоэквивалентность. Предположим, что существует изоморфизм функторов $f:\left.j \stackrel{\sim}{\longrightarrow} F\right|_{\mathscr{C}}$. Тогда этот изоморфизм может бить продолжен до изоморфизма id $\stackrel{\sim}{\longrightarrow} F$ на всю категорию $\mathbf{D}^{b}(\mathscr{A})$.

ДокАЗАТЕльство. Во-первых, так как $F$ коммутирует с прямыми суммами, то можно продолжить преобразование $f$ на все прямые суммы объектов категории $\mathscr{C}$ покомпонентно.

Отметим, что объект $X \in \mathbf{D}^{b}(\mathscr{A})$ изоморфен объекту из $\mathscr{A}$ тогда и только тогда, когда $\operatorname{Hom}^{j}\left(P_{i}, X\right)=0$ при $j \neq 0$ и всех $i \ll 0$. Отсюда следует, что объект $F(X)$ в этом случае также изоморфен объекту из $\mathscr{A}$, поскольку

$$
\operatorname{Hom}^{j}\left(P_{i}, F(X)\right) \cong \operatorname{Hom}^{j}\left(F\left(P_{i}\right), F(X)\right) \cong \operatorname{Hom}^{j}\left(P_{i}, X\right)=0
$$

при $j \neq 0$ и всех $i \ll 0$. 
Шаг 1. Пусть теперь $X$ - объект из категории $\mathscr{A}$. Зафиксируем сюръективньй морфизм $v: P_{i}^{\oplus k} \longrightarrow X$. Сушествуют изоморфизм $f_{i}: P_{i}^{\oplus k} \stackrel{\sim}{\longrightarrow} F\left(P_{i}^{\oplus k}\right)$ и два выделенных треугольника:

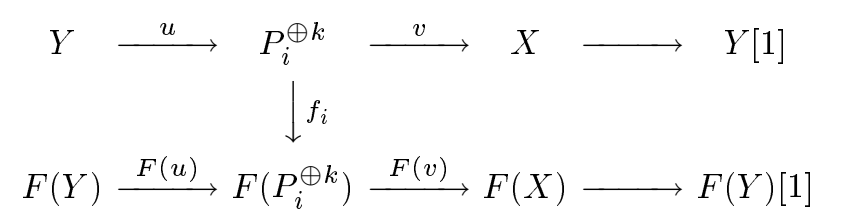

Давайте проверим, что $F(v) \circ f_{i} \circ u=0$. Для этого рассмотрим сюръективный морфизм $w: P_{j}^{\oplus l} \longrightarrow Y$. Достаточно проверить, что $F(v) \circ f_{i} \circ u \circ w=0$. Пусть $f_{j}: P_{j}^{\oplus l} \stackrel{\sim}{\longrightarrow} F\left(P_{j}^{\oplus l}\right)-$ канонический изоморфизм. Используя коммутационные соотношения для $f_{i}$ и $f_{j}$, имеем равенства

$$
F(v) \circ f_{i} \circ u \circ w=F(v) \circ F(u \circ w) \circ f_{j}=F(v \circ u \circ w) \circ f_{j}=0 .
$$

Так как $\operatorname{Hom}(Y[1], F(X))=0$, то по лемме 3.1.1 существует единственньй морфизм $f_{X}: X \longrightarrow F(X)$, который коммутирует с $f_{i}$.

Теперь рассмотрим конус $C_{X}$ морфизма $f_{X}$. Используя изоморфизмы

$$
\operatorname{Hom}\left(P_{i}, X\right) \cong \operatorname{Hom}\left(F\left(P_{i}\right), F(X)\right) \cong \operatorname{Hom}\left(P_{i}, F(X)\right)
$$

находим, что $\operatorname{Hom}^{j}\left(P_{i}, C_{X}\right)=0$ для всех $j$ и $i \ll 0$. Следовательно, по лемме 3.4 .3 $C_{X}=0$ и $f_{X}$ является изоморфизмом.

Шаг 2. Теперь покажем, что $f_{X}$ не зависит от выбора накрытия $v: P_{i}^{\oplus k} \longrightarrow X$. Рассмотрим два таких сюръективных морфизма $v_{1}: P_{i_{1}}^{\oplus k_{1}} \longrightarrow X$ и $v_{2}: P_{i_{2}}^{\oplus k_{2}} \longrightarrow X$. Всегда можно подобрать два таких сюръективных морфизма $w_{1}: P_{j}^{\oplus l} \longrightarrow P_{i_{1}}^{\oplus k_{1}}$ и $w_{2}: P_{j}^{\oplus l} \longrightarrow P_{i_{2}}^{\oplus k_{2}}$, что следующая диаграмма коммутативна:

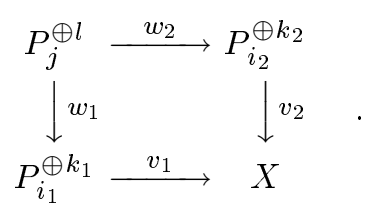

Очевидно, что достаточно проверить совпадение преобразований $f_{X}$, построенных по $v_{1}$ и $v_{1} \circ w_{1}$. Для этого рассмотрим коммутативную диаграмму:

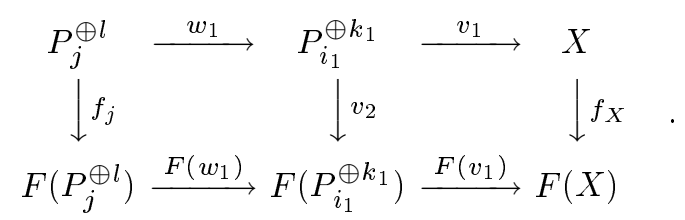

Здесь изоморфизм $f_{X}$ построен по $v_{1}$. Оба квадрата этой диаграммы коммутативны. Так как сушествует только один морфизм из $X$ в $F(X)$, который коммутирует с $f_{j}$, то $f_{X}$, построенньй по $v_{1}$, совпадает с морфизмом, построенным по $v_{1} \circ w_{1}$. 
Шаг 3. Теперь необходимо проверить, что морфизмы $f_{X}$ задают естественное преобразование функторов на категории $\mathscr{A}$. То есть нужно показать, что для любого морфизма $X \stackrel{\varphi}{\longrightarrow} Y$ имеется равенство

$$
f_{Y} \circ \varphi=F(\varphi) \circ f_{X}
$$

Рассмотрим сюръективньй морфизм $P_{j}^{\oplus l} \stackrel{v}{\longrightarrow} Y$. Подберем теперь индекс $i \ll 0$ и сюръективньй морфизм $P_{i}^{\oplus k} \stackrel{u}{\longrightarrow} X$ такие, что композиция $\varphi \circ u$ поднимается до морфизма $\psi: P_{i}^{\oplus k} \longrightarrow P_{j}^{\oplus l}$. Это можно сделать, поскольку при $i \ll 0$ отображение $\operatorname{Hom}\left(P_{i}^{\oplus k}, P_{j}^{\oplus l}\right) \rightarrow \operatorname{Hom}\left(P_{i}^{\oplus k}, Y\right)$ сюръективно. Получаем коммутативньй квадрат:

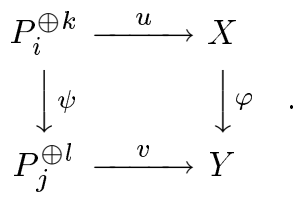

Обозначим через $h_{1}$ и $h_{2}$ композиции $f_{Y} \circ \varphi$ и $F(\varphi) \circ f_{X}$ соответственно. Имеем равенства:

$$
h_{1} \circ u=f_{Y} \circ \varphi \circ u=f_{Y} \circ v \circ \psi=F(v) \circ f_{j} \circ \psi=F(v) \circ F(\psi) \circ f_{i}
$$

И

$h_{2} \circ u=F(\varphi) \circ f_{X} \circ u=F(\varphi) \circ F(u) \circ f_{i}=F(\varphi \circ u) \circ f_{i}=F(v \circ \psi) \circ f_{i}=F(v) \circ F(\psi) \circ f_{i}$.

Таким образом, морфизмы $h_{t}$ при $t=1,2$ делают следующую диаграмму коммутативной:

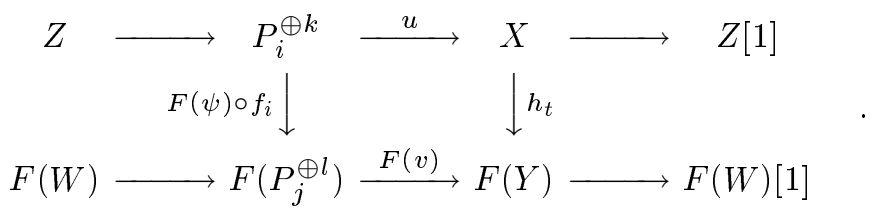

Так как $\operatorname{Hom}(Z[1], F(Y))=0$, то по лемме 3.1.1 получаем, что $h_{1}=h_{2}$. И, значит, $f_{Y} \circ \varphi=F(\varphi) \circ f_{X}$.

Шаг 4. Определим преобразование $f_{X[n]}: X[n] \longrightarrow F(X[n]) \cong F(X)[n]$ для каждого $X \in \mathscr{A}$ по формуле

$$
f_{X[n]}=f_{X}[n] .
$$

Нетрудно показать, что определенные таким образом преобразования коммутируют с любым морфизмом $u \in \operatorname{Ext}^{k}(X, Y)$. Действительно, всякий элемент $u \in \operatorname{Ext}^{k}(X, Y)$ может быть представлен как композиция $u=u_{0} u_{1} \cdots u_{k}$ некоторых элементов $u_{i} \in \operatorname{Ext}^{1}\left(Z_{i}, Z_{i+1}\right)$, где $Z_{0}=X, Z_{k}=Y$. Значит, достаточно проверить коммутирование с элементами $u \in \operatorname{Ext}^{1}(X, Y)$. Для этого рассмотрим диаграмму:

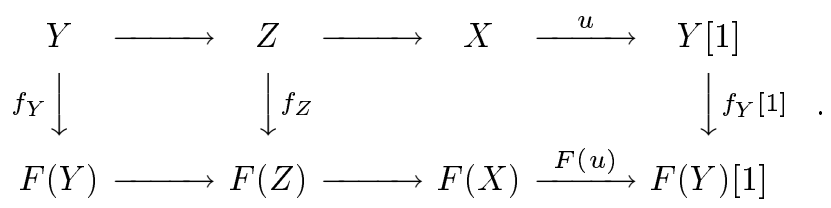


По одной из аксиом триангулированной категории найдется морфизм $h: X \rightarrow F(X)$ такой, что $\left(f_{Y}, f_{Z}, h\right)$ есть морфизм треугольников. С другой стороны, так как $\operatorname{Hom}(Y[1], F(X))=0$, то по лемме 3.1.1 морфизм $h$ определен однозначно тем условием, что коммутирует с $f_{Z}$. Но $f_{X}$ также коммутирует с $f_{Z}$. Следовательно, получаем, что $h=f_{X}$, и, значит,

$$
f_{Y}[1] \circ u=F(u) \circ f_{X}
$$

Шаг 5. Заключительную часть доказательства будем вести индукцией по длине отрезка, в котором находятся нетривиальные когомологии объекта. Для этого рассмотрим полную подкатегорию $j_{n}: \mathscr{D}_{n} \hookrightarrow \mathbf{D}^{b}(\mathscr{A})$ в $\mathbf{D}^{b}(\mathscr{A})$, состоящую из объектов, имеющих нетривиальные когомологии в некотором (нефиксированном) отрезке длины $n$. Теперь будем доказьвать, что существует единственное продолжение естественного преобразования $f$ до естественного функториального изоморфизма $f_{n}: j_{n} \longrightarrow$ $\left.F\right|_{\mathscr{D}_{n}}$.

Вьше это уже было показано для $n=1$, т.е. для основания индукции.

Теперь, чтобы сделать шаг индукции, предположим, что при $n=a, a \geqslant 1$, утверждение уже доказано. Пусть $X$ - некоторьй объект из $\mathscr{D}_{a+1}$ и положим, для определенности, что когомологии $H^{p}(X)$ являются нетривиальньми при $p \in[-a, 0]$. Возьмем $P_{i}$ из обильной последовательности с достаточно отрицательным номером $i$ такой, что

a) $\operatorname{Hom}^{j}\left(P_{i}, H^{p}(X)\right)=0$ для всех $p$ и для $j \neq 0$,

b) существует сюръективньй морфизм $u: P_{i}^{\oplus k} \longrightarrow H^{0}(X)$,

c) $\operatorname{Hom}\left(H^{0}(X), P_{i}\right)=0$.

Условие а) и стандартная спектральная последовательность дают нам изоморфизм $\operatorname{Hom}\left(P_{i}, X\right) \stackrel{\sim}{\longrightarrow} \operatorname{Hom}\left(P_{i}, H^{0}(X)\right)$. И, значит, можно найти морфизм $v: P_{i}^{\oplus k} \longrightarrow X$ такой, что композищия $v$ с каноническим морфизмом $X \longrightarrow H^{0}(X)$ совпадает с $u$. Рассмотрим выделенньй треугольник:

$$
Y[-1] \longrightarrow P_{i}^{\oplus k} \stackrel{v}{\longrightarrow} X \longrightarrow Y .
$$

Так как объект $Y$ принадлежит $\mathscr{D}_{a}$, то по предположению индукции изоморфизм $f_{Y}$ уже существует и коммутирует с $f_{i}$. Имеем диаграмму:

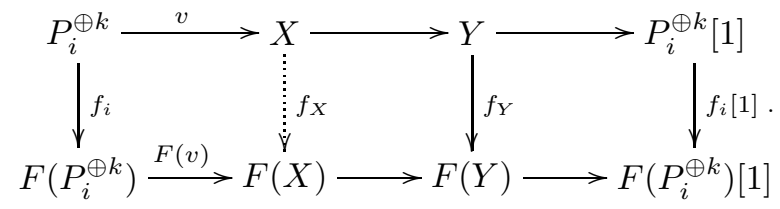

Далее, последовательность изоморфизмов

$$
\operatorname{Hom}\left(X, F\left(P_{i}^{\oplus k}\right)\right) \cong \operatorname{Hom}\left(X, P_{i}^{\oplus k}\right) \cong \operatorname{Hom}\left(H^{0}(X), P_{i}^{\oplus k}\right)=0
$$

дает нам возможность применить лемму $3.1 .1 \mathrm{k} g$, равному $f_{Y}$, из которой следует, что сушествует единственный морфизм $f_{X}: X \longrightarrow F(X)$, дополняющий диаграмму до морфизма треугольников. Очевидно, что $f_{X}$ на самом деле является изоморфизмом, так как изоморфизмами являются $f_{i}$ и $f_{Y}$. 
Шаг 6. Теперь необходимо показать, что $f_{X}$ не зависит от выбора $i$ и $u$. Пусть даны два сюръективных морфизма $u_{1}: P_{i_{1}}^{\oplus k_{1}} \longrightarrow H^{0}(X)$ и $u_{2}: P_{i_{2}}^{\oplus k_{2}} \longrightarrow H^{0}(X)$, удовлетворяюшие условиям (a), b) и с). Тогда можно подобрать достаточно отрицательное $j$ и сюръективные морфизмы $w_{1}, w_{2}$, делаюшие коммутативной следуюшую диаграмMy:

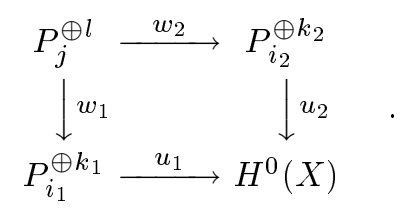

Обозначим через $v_{1}: P_{i_{1}}^{\oplus k_{1}} \longrightarrow X, v_{2}: P_{i_{2}}^{\oplus k_{2}} \longrightarrow X$ морфизмы, соответствуюшие $u_{1}$ и $u_{2}$. Так как $\operatorname{Hom}\left(P_{j}, X\right) \stackrel{\sim}{\longrightarrow} \operatorname{Hom}\left(P_{j}, H^{0}(X)\right)$, то получаем, что $v_{2} w_{2}=v_{1} w_{1}$.

Существует морфизм $\varphi: Y_{j} \longrightarrow Y_{i_{1}}$ такой, что тройка $\left(w_{1}, \mathrm{id}, \varphi\right)$ является морфизмом треугольников:

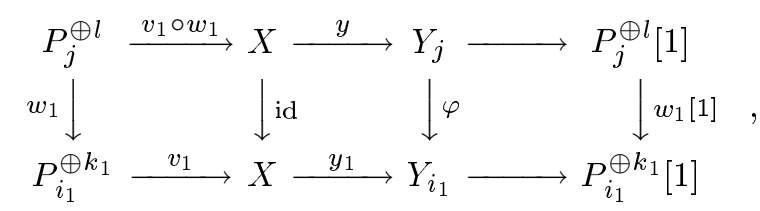

т.е. $\varphi y=y_{1}$.

Так как $Y_{j}$ и $Y_{i_{1}}$ имеют нетривиальные когомологии только в отрезке $[-a,-1]$, то по предположению индукции имеем коммутативньй квадрат:

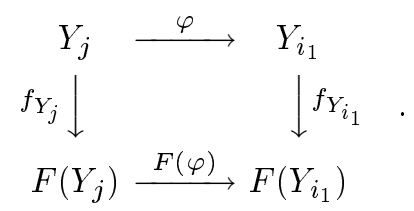

Обозначим через $f_{X}^{j}, f_{X}^{i_{1}}, f_{X}^{i_{2}}$ морфизмы, построенные по правилу, описанному выше, которые дополняют до коммутативной диаграмму (39) при $v$, соответственно равном $v=v_{1} w_{1}, v=v_{1}, v=v_{2}$. Как было уже доказано вьше (лемма 3.1.1), морфизм $f_{X}^{i_{1}}$ однозначно определен условием

$$
F\left(y_{1}\right) f_{X}^{i_{1}}=f_{Y_{i_{1}}} y_{1}
$$

С другой стороны, имеем равенства:

$$
F\left(y_{1}\right) f_{X}^{j}=F(\varphi y) f_{X}^{j}=F(\varphi) F(y) f_{X}^{j}=F(\varphi) F_{Y_{j}} y=f_{Y_{i_{1}}} \varphi y=f_{Y_{i_{1}}} y_{1}
$$

которые немедленно дают $f_{X}^{j}=f_{X}^{i_{1}}$. Аналогично получаем $f_{X}^{j}=f_{X}^{i_{2}}$. Таким образом, морфизм $f_{X}$ не зависит от выбора $i$ и морфизма $u: P_{i}^{\oplus k} \longrightarrow H^{0}(X)$ и, значит, определен абсолютно однозначно. 
Шаг 7. Таким образом получено некоторое продолжение преобразования $f_{a}$ на категорию $\mathscr{D}_{a+1}$. Нам осталось доказать, что это продолжение также является естественным преобразованием из $j_{a+1}$ в $\left.F\right|_{\mathscr{D}_{a+1}}$, т.е. что для всякого морфизма $\varphi: X \longrightarrow Y$, где $X, Y$ из $\mathscr{D}_{a+1}$, получается коммутативная диаграмма:

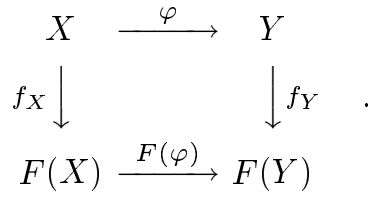

Сведем эту проблему к ситуации, когда оба объекта $X$ и $Y$ принадлежат $\mathscr{D}_{a}$.

Существует два случая.

Случай 1. Рассмотрим случай, когда старшая нетривиальная когомология объекта $X$ (без потери общности можно предполагать, что ее индекс равен 0) имеет индекс строго больший чем у объекта $Y$. Возьмем сюръективньй морфизм $u: P_{i}^{\oplus k}$ $H^{0}(X)$, удовлетворяюший условиям a), b), c), и построим, как и вьше, поднятие его до морфизма $v: P_{i}^{\oplus k} \longrightarrow X$. Имеем выделенный треугольник:

$$
P_{i}^{\oplus k} \stackrel{v_{1}}{\longrightarrow} X \stackrel{\alpha}{\longrightarrow} Z \longrightarrow P_{i}^{\oplus k}[1]
$$

Если выбрать $i$ достаточно отрицательным, то $\operatorname{Hom}\left(P_{i}^{\oplus k}, Y\right)=0$. Применяя функтор $\operatorname{Hom}(-, Y)$ к этому треугольнику, находим, что существует морфизм $\psi: Z \longrightarrow Y$ такой, что $\varphi=\psi \alpha$. Известно, что $f_{X}$, построенный ранее, удовлетворяет соотношению

$$
F(\alpha) f_{X}=f_{Z} \alpha
$$

Если предположить, что

$$
F(\psi) f_{Z}=f_{Y} \psi
$$

тогда получится, что

$$
F(\varphi) f_{X}=F(\psi) F(\alpha) f_{X}=F(\psi) f_{Z} \alpha=f_{Y} \psi \alpha=f_{Y} \varphi
$$

Это значит, что для проверки коммутативности квадрата (40) можно заменить $X$ на $Z$. А верхняя грань нетривиальных когомологий для $Z$ на единицу меньше, чем у $X$. Более того, видно, что если $X$ принадлежит категории $\mathscr{D}_{k}$ с $k>1$, то $Z$ принадлежит $\mathscr{D}_{k-1}$, а если $X$ принадлежит $\mathscr{D}_{1}$, то $Z$ также принадлежит $\mathscr{D}_{1}$, но номер его нетривиальной когомологии на единицу меньше.

Случай 2. Теперь рассмотрим другой случай: старшая нетривиальная когомология $Y$ (опять можем считать, что ее номер равен 0 ) имеет номер не меньше чем у $X$. Возьмем сюръективньй морфизм $u: P_{i}^{\oplus k} \longrightarrow H^{0}(Y)$, удовлетворяющий условиям a), b), c), и построим морфизм $v: P_{i}^{\oplus k} \longrightarrow Y$, который однозначно определен морфизмом $u$. Рассмотрим выделенный треугольник:

$$
P_{i}^{\oplus k} \stackrel{v}{\longrightarrow} Y \stackrel{\beta}{\longrightarrow} W \longrightarrow P_{i}^{\oplus k}
$$

Обозначим через $\psi$ композицию $\beta \circ \varphi$. 
Если теперь предположить, что

$$
F(\psi) f_{X}=f_{W} \psi
$$

тогда, так как $F(\beta) f_{Y}=f_{W} \beta$, будем иметь:

$$
F(\beta)\left(f_{Y} \varphi-F(\varphi) f_{X}\right)=f_{W} \beta \varphi-f(\beta \varphi) f_{X}=f_{W} \psi-F(\psi) f_{X}=0
$$

Снова выберем $i$ достаточно отрицательным, так чтобы выполнялось зануление $\operatorname{Hom}\left(X, P_{i}^{\oplus k}\right)=0$. Так как объект $F\left(P_{i}^{\oplus k}\right)$ изоморфен объекту $P_{i}^{\oplus k}$, то имеем равенство $\operatorname{Hom}\left(X, F\left(P_{i}^{\oplus k}\right)\right)=0$. Теперь, применяя функтор $\operatorname{Hom}(X, F(-))$ к треугольнику (41), находим, что $F(\beta)$ задает вложение пространства $\operatorname{Hom}(X, F(Y))$ в $\operatorname{Hom}(X, F(W))$. Из равенства (42) теперь сразу следует равенство $f_{Y} \varphi=F(\varphi) f_{X}$.

Таким образом, чтобы проверить коммутативность квадрата (40), можно заменить $Y$ на объект $W$, верхняя граница нетривиальных когомологий которого на единищу меньше, чем у $Y$. Если $Y$ принадлежал $\mathscr{D}_{k}, k>1$, то $W$ принадлежит уже $\mathscr{D}_{k-1}$. Если $Y$ принадлежал $\mathscr{D}_{1}$, то объект $W$ также принадлежит $\mathscr{D}_{1}$, но номер его нетривиальной когомологии на единицу меньше.

Предположим сейчас, что объекты $X$ и $Y$ лежат в категории $\mathscr{D}_{a+1}, a>1$. В зависимости от того, какой из двух случаев, 1) или 2), применим, можно заменить либо $X$, либо $Y$ на объект, лежаший уже в категории $\mathscr{D}_{a}$. Повторяя, если необходимо, эту процедуру, можно уменьшить верхнюю границу когомологий этого объекта так, что другой случай будет применим. Тогда можно будет уменьшить длину нетривиальных когомологий у второго объекта и прийти к ситуации, когда оба объекта уже лежат в категории $\mathscr{D}_{a}$. Таким образом делается шаг индукции.

В заключение отметим, что при построении изоморфизмов $f_{X}$ они всегда были определены однозначно. Это значит, что естественное преобразование из id в $F$, которое было построено, является единственным. Предложение доказано.

ДоКАЗАТЕЛЬСТВо ТЕОРЕмЫ 3.2.1. 1) Сушествование. Предложение 3.3.5 и лемма 3.3.1 позволяют по функтору $F$ построить объект $\mathscr{E} \in \mathbf{D}^{b}(M \times X)$ такой, что сушествует изоморфизм функторов $\bar{f}:\left.\left.F\right|_{\mathscr{C}} \stackrel{\sim}{\longrightarrow} \Phi_{\mathscr{E}}\right|_{\mathscr{C}}$ на полной подкатегории $\mathscr{C} \subset$ $\mathbf{D}^{b}(M)$, где $\mathrm{Ob} \mathscr{C}=\left\{\mathscr{L}^{i} \mid i \in \mathbb{Z}\right\}$ и $\mathscr{L}$ - очень обильное расслоение на $M$, для которого $\mathrm{H}^{i}\left(M, \mathscr{L}^{k}\right)=0$ при $k>0$ и $i \neq 0$.

По лемме 3.4 .5 функтор $\Phi_{\mathscr{E}}$ является вполне строгим. Кроме того, так как имеются изоморфиимы:

$$
\begin{gathered}
F^{!}(\bar{f}):\left.\left.F^{!} \circ F\right|_{\mathscr{C}} \cong \operatorname{id}_{\mathscr{C}} \stackrel{\sim}{\longrightarrow} F^{!} \circ \Phi_{\mathscr{E}}\right|_{\mathscr{C}}, \\
\Phi_{\mathscr{E}}^{*}(\bar{f}):\left.\left.\Phi_{\mathscr{E}}^{*} \circ F\right|_{\mathscr{C}} \stackrel{\sim}{\longrightarrow} \Phi_{\mathscr{E}}^{*} \circ \Phi_{\mathscr{E}}\right|_{\mathscr{C}} \cong \operatorname{id}_{\mathscr{C}},
\end{gathered}
$$

то опять по лемме 3.4 .5 функторы $F^{!} \circ \Phi_{\mathscr{E}}$ и $\Phi_{\mathscr{E}}^{*} \circ F$ также являются вполне строгими. А так как они сопряжены друг другу, то эти функторы $F^{!} \circ \Phi_{\mathscr{E}}$ и $\Phi_{\mathscr{E}}^{*} \circ F$ на самом деле являются эквивалентностями.

Рассмотрим снова изоморфизм $F^{!}(\bar{f}):\left.\left.F^{!} \circ F\right|_{\mathscr{C}} \cong \mathrm{id}_{\mathscr{C}} \stackrel{\sim}{\longrightarrow} F^{!} \circ \Phi_{\mathscr{E}}\right|_{\mathscr{C}}$ на подкатегории $\mathscr{C}$. По предложению 3.4.6 можно продолжить его до изоморфизма на всем $\mathbf{D}^{b}(M)$, т.e. id $\stackrel{\sim}{\longrightarrow} F^{!} \circ \Phi_{\mathscr{E}}$. 
Так как $F^{\text {! } я в л я е т с я ~ с о п р я ж е н н ы м ~ с п р а в а ~ к ~} F$, то получается морфизм функторов $f: F \longrightarrow \Phi_{\mathscr{E}}$, для которого $\left.f\right|_{\mathscr{C}}=\bar{f}$. Осталось проверить, что $f$ есть изоморфизм. Действительно, возьмем конус $C_{Z}$ канонического морфизма $f_{Z}: F(Z) \longrightarrow \Phi_{\mathscr{E}}(Z)$. Так как $F^{!}\left(f_{Z}\right)$ является изоморфизмом, получаем, что $F^{!}(Z)=0$. Следовательно, для любого объекта $Y$ имеем $\operatorname{Hom}\left(F(Y), C_{Z}\right)=0$. Далее, так как $F\left(\mathscr{L}^{k}\right) \cong \Phi_{\mathscr{E}}\left(\mathscr{L}^{k}\right)$ для всех $k$, получаем последовательность изоморфизмов

$$
\operatorname{Hom}^{i}\left(\mathscr{L}^{k}, \Phi_{\mathscr{E}}^{!}\left(C_{Z}\right)\right)=\operatorname{Hom}^{i}\left(\Phi_{\mathscr{E}}\left(\mathscr{L}^{k}\right), C_{Z}\right)=\operatorname{Hom}^{i}\left(F\left(\mathscr{L}^{k}\right), C_{Z}\right)=0
$$

для всех $k$ и $i$.

Применяя лемму 3.6 , сразу получаем равенство $\Phi_{\mathscr{E}}^{!}\left(C_{Z}\right)=0$. Следовательно, $\operatorname{Hom}\left(\Phi_{\mathscr{E}}(Z), C_{Z}\right)=0$. Это влечет, что треугольник для морфизма $f_{Z}$ должен расшепляться, т.е. $F(Z)=C_{Z}[-1] \oplus \Phi_{\mathscr{E}}(Z)$. Но, как уже было показано, $\operatorname{Hom}\left(F(Y), C_{Z}\right)=0$ для любого $Y$ и, значит, для $Z[1]$ также. А это может быть, только если $C_{Z}=0$, и $f_{Z}-$ изоморфизм.

2) Единственность. Единственность представляющего объекта на самом деле следует из построения, так как всякий раз имеется однозначность при построении объекта. Но давайте пройдем это еще раз. Предположим, что существуют два объекта $\mathscr{E}_{1}$ и $\mathscr{E}_{2}$ в категории $D^{b}(M \times X)$, для которых $\Phi_{\mathscr{E}_{1}} \cong F \cong \Phi_{\mathscr{E}_{2}}$. Рассмотрим функтор $F^{\prime}=\mathbf{L} j^{*} \circ F$, где, как и вьше, $j: M \longrightarrow \mathbb{P}^{N}$ - вложение с помошью подходящего очень обильного линейного расслоения. Объекты $\mathbf{R} J_{*} \mathscr{E}_{i}, i=1,2$, оба должны быть свертками комплекса (32)

$$
C^{\cdot}:=\left\{\mathscr{O}(-N) \otimes F^{\prime}\left(\Omega^{N}(N)\right) \stackrel{d_{-N}^{\prime}}{\longrightarrow} \cdots \longrightarrow \mathscr{O}(-1) \otimes F^{\prime}\left(\Omega^{1}(1)\right) \stackrel{d_{-1}^{\prime}}{\longrightarrow} \mathscr{O} \otimes F^{\prime}(\mathscr{O})\right\} .
$$

Но, как было показано вьше, все свертки этого комплекса изоморфны по лемме 3.1.2. Таким образом, $\mathbf{R} J_{*} \mathscr{E}_{1} \cong \mathbf{R} J_{*} \mathscr{E}_{2}$. Применяя теперь предложение 3.3 .5 , получаем, что и сами $\mathscr{E} 1$ и $\mathscr{E} 2$ также изоморфны.

3.5. Приложение: $n$-кошулевость однородной координатной алгебры. Факты, собранные в этом приложении, не являются оригинальными. В той или иной форме они известны. Однако, не имея хорошей ссылки, мы вынуждены привести собственное доказательство утверждения, которое используется в основном тексте и в той форме, которая нам необходима. Здесь в основном используется техника из статьи [18].

Пусть $X$ - гладкое проективное многообразие и $\mathscr{L}$ - очень обильное линейное расслоение на $X$, удовлетворяющее дополнительному условию $\mathrm{H}^{i}\left(X, \mathscr{L}^{k}\right)=0$ для всех $k>0$ и $i \neq 0$. Обозначим через $A$ координатную алгебру многообразия $X$ относительно расслоения $\mathscr{L}$, т.е. $A=\bigoplus_{k=0}^{\infty} \mathrm{H}^{0}\left(X, \mathscr{L}^{k}\right)$.

Рассмотрим многообразие $X^{n}$ для некоторого $n \in \mathbb{N}$. В дальнейшем будем обозначать через $\pi_{i}^{(n)}$ проекцию $X^{n}$ на $i$-ю компоненту, а через $\pi_{i j}^{(n)}-$ проекцию $X^{n}$ на произведение $i$-й и $j$-й компонент. Определим подмногообразия $\Delta_{\left(i_{1}, \ldots, i_{k}\right)\left(i_{k+1}, \ldots, i_{m}\right)}^{(n)} \subset X^{n}$ следуюшим образом:

$$
\Delta_{\left(i_{1}, \ldots, i_{k}\right)\left(i_{k+1}, \ldots, i_{m}\right)}^{(n)}:=\left\{\left(x_{1}, \ldots, x_{n}\right) \mid x_{i_{1}}=\cdots=x_{i_{k}} ; x_{i_{k+1}}=\cdots=x_{m}\right\} .
$$

Для краткости будем писать $S_{i}^{(n)}$ вместо $\Delta_{(n, \ldots, i)}^{(n)}$. Очевидно, что $S_{i}^{(n)} \cong X^{i}$. 
Теперь положим

$$
T_{i}^{(n)}:=\bigcup_{k=1}^{i-1} \Delta_{(n, \ldots, i)(k, k-1)}^{(n)}, \quad \Sigma^{(n)}:=\bigcup_{k=1}^{n} \Delta_{(k, k-1)}^{(n)} .
$$

(По определению $T_{1}^{(n)}$ и $T_{2}^{(n)}$-пустые подмножества.) Легко видеть, что $T_{i}^{(n)} \subset S_{i}^{(n)}$.

Обозначим через $J_{\Sigma^{(n)}}$ пучок идеалов подсхемы $\Sigma^{(n)} \subset X^{n}$, а через $\mathscr{I}_{i}^{(n)}-$ пучок на $X^{n}$, которьй является ядром естественного отображения $\mathscr{O}_{S_{i}^{(n)}} \longrightarrow \mathscr{O}_{T_{i}^{(n)}} \longrightarrow 0$.

Зафиксируем на время $m$ и $k \leqslant m$. Пусть $s-$ вложение подмногообразия $S_{k}^{(m)} \cong$ $X^{k-1} \times X$ в $X^{k}$, котороепо определению многообразия $S_{k}^{(m)}$ является тождественным по первым $k-1$ компонентам и диагонально по последней $k$-й компоненте. Обозначим через $p$ проекцию $S_{k}^{(m)}$ на $X^{k-1}$, которое есть произведение первых $k-1$ сомножителей.

ЛЕмма 3.5.1. Пучок $\mathscr{I}_{1}^{(m)}$ изоморфен пучку $\mathscr{O}_{\Delta_{(n, \ldots, 1)}^{(n)}}$, а при $k>1$ пучок $\mathscr{I}_{k}^{(m)}$ изоморфен $s_{*} p^{*}\left(J_{\Sigma^{(k-1)}}\right)$. В частности, при $k>1$ имеются изоморфизмы:

a) $\mathrm{H}^{j}\left(X^{m}, \mathscr{I}_{k}^{(m)} \otimes\left(\mathscr{L} \otimes \cdots \otimes \mathscr{L}^{i}\right)\right)$

$$
=\mathrm{H}^{j}\left(X^{k-1}, J_{\Sigma^{(k-1)}} \otimes(\mathscr{L} \otimes \cdots \otimes \mathscr{L})\right) \otimes A_{m-k+i} \quad \text { для } \quad \text { всех } i>0 ;
$$

b) $\quad \mathbf{R}^{j} \pi_{1 *}^{(m)}\left(\mathscr{I}_{k}^{(m)} \otimes\left(\mathscr{O} \otimes \mathscr{L} \otimes \cdots \otimes \mathscr{L}^{i}\right)\right)$

$$
\cong \mathbf{R}^{j} \pi_{1 *}^{(k-1)}\left(J_{\Sigma^{(k-1)}} \otimes(\mathscr{O} \otimes \mathscr{L} \otimes \cdots \otimes \mathscr{L})\right) \otimes A_{m-k+i} \quad \text { для } \quad \text { всех } i>0 ;
$$

c) $\quad \mathbf{R}^{j} \pi_{1 m *}^{(m)}\left(\mathscr{I}_{k}^{(m)} \otimes\left(\mathscr{O} \otimes \mathscr{L} \otimes \cdots \otimes \mathscr{L}^{i}\right)\right)$

$$
\cong \mathbf{R}^{j} \pi_{1 *}^{(k-1)}\left(J_{\Sigma^{(k-1)}} \otimes(\mathscr{O} \otimes \mathscr{L} \otimes \cdots \otimes \mathscr{L})\right) \otimes \mathscr{L}^{m-k+i} \quad \text { для всех } i .
$$

ДокАЗАТЕЛЬСтво. Утверждение про то, что при $k>1$ пучок $\mathscr{I}_{k}^{(m)}$ изоморфен $s_{*} p^{*}\left(J_{\Sigma^{(k-1)}}\right)$, сразу следует из определений пучка $\mathscr{I}_{k}^{(m)}$ и подсхем $T_{k}^{(m)}$ и $S_{k}^{(m)}$. Все остальное немедленное следствие этого факта.

Используя индукцию по $n$, несложно проверить, что следующий комплекс на $X^{n}$ :

$$
P_{n}^{\cdot}: 0 \longrightarrow J_{\Sigma^{(n)}} \longrightarrow \mathscr{I}_{n}^{(n)} \longrightarrow \mathscr{I}_{n-1}^{(n)} \longrightarrow \cdots \longrightarrow \mathscr{I}_{2}^{(n)} \longrightarrow \mathscr{I}_{1}^{(n)} \longrightarrow 0
$$

является точным. В качестве примера отметим, что при $n=2$ этот комплекс есть короткая точная последовательность на $X \times X$ :

$$
P_{2}^{:}: 0 \longrightarrow J_{\Delta} \longrightarrow \mathscr{O}_{X \times X} \longrightarrow \mathscr{O}_{\Delta} \longrightarrow 0 .
$$

Лемма 3.5.2. Пусть $X$ - гладкое проективное многообразие с обильнымм линейным расслоением $\mathscr{M}$. Тогда для любого натурального числа $k$ существует $i$ такое, что для расслоения $\mathscr{L}=\mathscr{M}^{i}$ и всех $1<m \leqslant k$ выполняются следующие свойства:

$$
\begin{aligned}
& \text { a) } \mathrm{H}^{j}\left(X^{m}, J_{\Sigma(m)} \otimes(\mathscr{L} \otimes \cdots \otimes \mathscr{L})\right)=0 \\
& \text { b) } \quad \mathbf{R}^{j} \pi_{1 *}^{(m)}\left(J_{\Sigma^{(m)}} \otimes(\mathscr{O} \otimes \mathscr{L} \otimes \cdots \otimes \mathscr{L})\right)=0 \quad \partial_{\Omega я} \quad j \neq 0 \text {; } \\
& \text { c) } \left.\quad \mathbf{R}^{j} \pi_{1 m *}^{(m)}\left(J_{\Sigma^{(m)}} \otimes(\mathscr{O} \otimes \mathscr{L} \otimes \cdots \otimes \mathscr{L} \otimes \mathscr{O})\right)=0 \quad \partial \Omega^{\prime} \quad j \neq 0 . \quad\right\}
\end{aligned}
$$


ДокАЗАТЕльство. Для любого $m$ линейные расслоения $\mathscr{M} \otimes \cdots \otimes \mathscr{M}, \mathscr{O} \otimes \mathscr{M} \otimes$ $\cdots \otimes \mathscr{M}$ и $\mathscr{O} \otimes \mathscr{M} \otimes \cdots \otimes \mathscr{M} \otimes \mathscr{O}$ на $X^{m}$ являются соответственно обильным, $\pi_{1}^{(m)}$ обильньм и $\pi_{1 m}^{(m)}$-обильным. Поэтому для каждого из них найдется целое число такое, что для всех степеней этих расслоений, больших данного числа, свойства а), b) и с) вьполнены. Возьмем максимальное среди этих чисел по всем $m \leqslant k$. Обозначим этот максимум через $i$. Тогда для $\mathscr{L}=\mathscr{M}^{i}$ свойства a), b) и с) также вьполнены.

Введем следуюшие обозначения:

$B_{n}:=\mathrm{H}^{0}\left(X^{n}, J_{\Sigma^{(n)}} \otimes(\mathscr{L} \otimes \cdots \otimes \mathscr{L})\right), \quad R_{n-1}:=\mathbf{R}^{0} \pi_{1 *}^{(n)}\left(J_{\Sigma^{(n)}} \otimes(\mathscr{O} \otimes \mathscr{L} \otimes \cdots \otimes \mathscr{L})\right)$

ПРЕДЛОЖЕНИЕ 3.5.3. Пусть $\mathscr{L}$ - очень обильное расслоение на гладком проективном многообразии $X$, которое удовлетворяет условию (43) для всех таких, что $1<m \leqslant n+\operatorname{dim} X+2$. Тогда

1) алгебра $А$ является $n$-кошулевой, т.е. последовательность

$$
B_{n} \otimes_{k} A \longrightarrow B_{n-1} \otimes_{k} A \longrightarrow \cdots \longrightarrow B_{1} \otimes_{k} A \longrightarrow A \longrightarrow k \longrightarrow 0
$$

точна:

2) комплекс пучков на $X$

$A_{k-n} \otimes R_{n} \longrightarrow A_{k-n+1} \otimes R_{n-1} \longrightarrow \cdots \longrightarrow A_{k-1} \otimes R_{1} \longrightarrow A_{k} \otimes R_{0} \longrightarrow \mathscr{L}^{k} \longrightarrow 0$

точен для всех $k \geqslant 0\left(\right.$ если $k-i<0$, тогда по определению $\left.A_{k-i}=0\right)$;

3) комплекс пучков на $X \times X$

$$
\mathscr{L}^{-n} \otimes R_{n} \longrightarrow \cdots \longrightarrow \mathscr{L}^{-1} \otimes R_{1} \longrightarrow \mathscr{O}_{M} \otimes R_{0} \longrightarrow \mathscr{O}_{\Delta}
$$

является точным т.е. дает п-резольвенту диагонали на $X \times X$.

ДокАЗАТЕЛЬСТво. 1) Во-первых, комбинируя леммы 3.5 .1 и 3.5.2, для всех $1<$ $m \leqslant n+\operatorname{dim} X+2$ получаем:

1) $\mathrm{H}^{0}\left(X^{m}, \mathscr{I}_{k}^{(m)} \otimes\left(\mathscr{L} \otimes \cdots \otimes \mathscr{L}^{i}\right)\right)$

$$
=\mathrm{H}^{0}\left(X^{k-1}, J_{\Sigma^{(k-1)}} \otimes(\mathscr{L} \otimes \cdots \otimes \mathscr{L})\right) \otimes A_{m-k+i}=B_{k-1} \otimes A_{m-k+i} ;
$$

2) $\mathrm{H}^{j}\left(X^{m}, \mathscr{I}_{k}^{(m)} \otimes\left(\mathscr{L} \otimes \cdots \otimes \mathscr{L}^{i}\right)\right)=0$ при $j \neq 0$.

Рассмотрим комплексы $P_{m}^{\cdot} \otimes(\mathscr{L} \otimes \cdots \otimes \mathscr{L})$ при $m \leqslant n+\operatorname{dim} X+1$. Применяя к ним функтор $\mathrm{H}^{0}$ и используя условие (44), получаем точные последовательности:

$$
0 \longrightarrow B_{m} \longrightarrow B_{m-1} \otimes_{k} A_{1} \longrightarrow \cdots \longrightarrow B_{1} \otimes_{k} A_{m-1} \longrightarrow A_{m} \longrightarrow 0
$$

для всех $m \leqslant n+\operatorname{dim} X+1$.

Теперь положим $m=n+\operatorname{dim} X+1$ и обозначим через $W_{m}$ комплекс

$$
\mathscr{I}_{m}^{(m)} \longrightarrow \mathscr{I}_{m-1}^{(m)} \longrightarrow \cdots \longrightarrow \mathscr{I}_{2}^{(m)} \longrightarrow \mathscr{I}_{1}^{(m)} \longrightarrow 0
$$


которьй является правой резольвентой пучка $J_{\Sigma^{(m)}}$. Возьмем комплекс

$$
W_{m}^{\cdot} \otimes\left(\mathscr{L} \otimes \cdots \otimes \mathscr{L} \otimes \mathscr{L}^{i}\right)
$$

и применим к нему функтор $\mathrm{H}^{0}$. Получим последовательность

$$
B_{m-1} \otimes_{k} A_{i} \longrightarrow B_{m-2} \otimes_{k} A_{i+1} \longrightarrow \cdots \longrightarrow B_{1} \otimes_{k} A_{m+i-2} \longrightarrow A_{m+i-1} \longrightarrow 0 .
$$

Из свойства (44) 2) следует, что когомологии этого комплекса есть $\mathrm{H}^{j}\left(X^{m}, J_{\Sigma(m)} \otimes\right.$ $\left.\left(\mathscr{L} \otimes \cdots \otimes \mathscr{L} \otimes \mathscr{L}^{i}\right)\right)$. Из условия (43) b) следует, что

$$
\begin{aligned}
& \mathrm{H}^{j}\left(X^{m}, J_{\Sigma^{(m)}} \otimes\left(\mathscr{L} \otimes \cdots \otimes \mathscr{L} \otimes \mathscr{L}^{i}\right)\right) \\
& \quad=\mathrm{H}^{j}\left(X, \mathbf{R}^{0} \pi_{m *}^{(m)}\left(J_{\Sigma^{(m)}} \otimes(\mathscr{L} \otimes \cdots \otimes \mathscr{L} \otimes \mathscr{O})\right) \otimes \mathscr{L}^{i}\right) .
\end{aligned}
$$

Следовательно, они тривиальны при $j>\operatorname{dim} X$, и, значит, имеется точная последовательность:

$B_{n} \otimes_{k} A_{m-n+i-1} \longrightarrow B_{n-1} \otimes_{k} A_{m-n+i} \longrightarrow \cdots \longrightarrow B_{1} \otimes_{k} A_{m+i-2} \longrightarrow A_{m+i-1} \longrightarrow 0$

при $i \geqslant 1$. А при $i \leqslant 1$ точность была доказана ранее. То есть алгебра $A$ является $n$-кошулевой.

2) Доказательство аналогично доказательству пункта 1). Имеем изоморфизмы:

1) $\mathbf{R}^{0} \pi_{1 *}^{(m)}\left(\mathscr{I}_{k}^{(m)} \otimes(\mathscr{O} \otimes \mathscr{L} \otimes \cdots \otimes \mathscr{L})\right)$

$$
\cong \mathbf{R}^{0} \pi_{1 *}^{(k-1)}\left(J_{\Sigma^{(k-1)}} \otimes(\mathscr{O} \otimes \mathscr{L} \otimes \cdots \otimes \mathscr{L})\right) \otimes A_{m-k+1}
$$

2) $\mathbf{R}^{j} \pi_{1 *}^{(m)}\left(\mathscr{I}_{k}^{(m)} \otimes(\mathscr{O} \otimes \mathscr{L} \otimes \cdots \otimes \mathscr{L})\right)=0$ для всех $j \neq 0$.

Применяя теперь функтор $\mathbf{R}^{0} \pi_{1 *}^{(m)}$ к комплексам $P_{m}^{\cdot} \otimes(\mathscr{O} \otimes \mathscr{L} \otimes \cdots \otimes \mathscr{L})$ при $m \leqslant n+$ $\operatorname{dim} X+2$, получаем точньй комплекс пучков на $X$ :

$0 \longrightarrow R_{m-1} \longrightarrow A_{1} \otimes R_{m-2} \longrightarrow \cdots \longrightarrow A_{m-2} \otimes R_{1} \longrightarrow A_{m-1} \otimes R_{0} \longrightarrow \mathscr{L}^{m-1} \longrightarrow 0$ для $m \leqslant n+\operatorname{dim} X+2$.

Рассмотрим $m=n+\operatorname{dim} X+2$. Применяя функтор $\mathbf{R}^{0} \pi_{1 *}^{(m)}$ к комплексу

$$
W_{m}^{\cdot} \otimes\left(\mathscr{O} \otimes \mathscr{L} \otimes \cdots \otimes \mathscr{L} \otimes \mathscr{L}^{i}\right)
$$

получаем комплекс

$$
A_{i} \otimes R_{m-2} \longrightarrow \cdots \longrightarrow A_{m+i-3} \otimes R_{1} \longrightarrow A_{m+i-2} \otimes R_{0} \longrightarrow \mathscr{L}^{m+i-2} \longrightarrow 0 .
$$

По свойству (45) когомологии этого комплекса суть

$$
\begin{aligned}
& \mathbf{R}^{j} \pi_{1 *}^{(m)}\left(J_{\Sigma^{(m)}} \otimes\left(\mathscr{O} \otimes \mathscr{L} \otimes \cdots \otimes \mathscr{L} \otimes \mathscr{L}^{i}\right)\right) \\
& \quad \cong \mathbf{R}^{j} p_{1 *}\left(\mathbf{R}^{0} \pi_{1 m *}^{(m)}\left(J_{\Sigma^{(m)}} \otimes(\mathscr{O} \otimes \mathscr{L} \otimes \cdots \otimes \mathscr{L} \otimes \mathscr{O})\right) \otimes\left(\mathscr{O} \otimes \mathscr{L}^{i}\right)\right)
\end{aligned}
$$


которые тривиальны при $j>\operatorname{dim} X$. Значит последовательность пучков на $X$ $A_{k-n} \otimes R_{n} \longrightarrow A_{k-n+1} \otimes R_{n-1} \longrightarrow \cdots \longrightarrow A_{k-1} \otimes R_{1} \longrightarrow A_{k} \otimes R_{0} \longrightarrow \mathscr{L}^{k} \longrightarrow 0$ точна для всех $k \geqslant 0$.

3) Рассмотрим комплекс $W_{n+2}^{\cdot} \otimes\left(\mathscr{O} \otimes \mathscr{L} \otimes \cdots \otimes \mathscr{L} \otimes \mathscr{L}^{-n}\right)$ на $X^{n+2}$. Применяя теперь функтор $\mathbf{R}^{0} \pi_{1(n+2) *}^{(n+2)}$ к нему, получаем следуюший комплекс на $X \times X$ :

$$
\mathscr{L}^{-n} \otimes R_{n} \longrightarrow \cdots \longrightarrow \mathscr{L}^{-1} \otimes R_{1} \longrightarrow \mathscr{O}_{M} \otimes R_{0} \longrightarrow \mathscr{O}_{\Delta}
$$

Вспоминая условие с) леммы 3.5.1 и условие (43) b), находим, что когомологии этого комплекса изоморфны пучкам

$$
\mathbf{R}^{j} \pi_{1(n+2) *}^{(n+2)}\left(J_{\Sigma^{(n+2)}} \otimes(\mathscr{O} \otimes \mathscr{L} \otimes \cdots \otimes \mathscr{L} \otimes \mathscr{O})\right) \otimes\left(\mathscr{O} \otimes \mathscr{L}^{-n}\right),
$$

которые по свойству (43) с) равны 0 при $j>0$. То есть комплекс (46) является точным.

\section{Глава 4. Производные категории когерентных пучков на К3 поверхностях}

4.1. К3 поверхности и решетка Мукаи. Эта глава целиком посвящена производным категориям когерентных пучков на поверхностях типа К3 над полем комплексных чисел. Основной вопрос, который нас интересует и на который дается ответ в этой главе: когда две разных К3 поверхности имеют эквивалентные категории когерентных пучков? Как и раньше, производные категории рассматриваются как триангулированные и эквивалентности понимаются как эквивалентности между триангулированными категориями.

Напомним, что для гладких проективных многообразий с обильным каноническим или антиканоническим классом существует процедура восстановления производной категории когерентных пучков (см. теорему 2.1.3). Однако для многообразий другого типа вопрос этот нетривиальный и особенно интересен для многообразий с тривиальным каноническим классом.

Начнем с основных необходимых нам фактов про К3 поверхности. Напомним, что поверхность типа К3 - это компактная гладкая алгебраическая поверхность $S$, для которой $K_{S}=0$ и $\mathrm{H}^{1}(S, \mathbb{Z})=0$. Такие поверхности на самом деле являются односвязными. Можно показать, что вторые когомологии $\mathrm{H}^{2}(S, \mathbb{Z})$ не имеют кручения и являются четной решеткой ранга 22 относительно формы пересечения. Кроме того, из формулы Нётера следует, что $p_{g}(S)=1$ и $h^{1,1}(S)=20$.

Одним из основных инвариантов поверхности типа К3 является ее группа Нерона-Севери $\mathrm{NS}(S) \subset \mathrm{H}^{2}(S, \mathbb{Z})$, которая в этом случае совпадает с группой Пикара $\operatorname{Pic}(S)$. Ранг решетки $\operatorname{NS}(S)$ не превосходит $h^{1,1}=20$. Обозначим через $\mathrm{T}_{S}$ решетку трансцедентных циклов, которая по определению есть ортогональное дополнение к решетке Нерона-Севери $\operatorname{NS}(S)$ во вторых когомологиях $\mathrm{H}^{2}(S, \mathbb{Z})$.

Теперь, обозначим через $t d_{S}$ класс Тодда поверхности $S$, которьй есть элемент $\mathrm{H}^{*}(S, \mathbb{Q})$. Он имеет вид $1+2 w$, где $1 \in \mathrm{H}^{0}(S, \mathbb{Z})$ - это единица кольца когомологий $\mathrm{H}^{*}(S, \mathbb{Z})$ и $w \in \mathrm{H}^{4}(S, \mathbb{Z})$ - фундаментальньй коцикл $S$. Рассмотрим положительньй 
квадратный корень $\sqrt{t d_{S}}=1+w$, который для К3 поверхности лежит в кольце целых когомологий $\mathrm{H}^{*}(S, \mathbb{Z})$.

Для любого когерентного пучка на $S$ определен характер Черна, которьй по аддитивности продолжается на всю производную категорию когерентных пучков. Если $F$ - объект $\mathbf{D}^{b}(S)$, то характер Черна

$$
\operatorname{ch}(F)=r(F)+c_{1}(F)+\frac{1}{2}\left(c_{1}^{2}-2 c_{2}\right)
$$

принадлежит целым когомологиям $\mathrm{H}^{*}(S, \mathbb{Z})$. Для любого объекта $F$ определим элемент

$$
v(F)=\operatorname{ch}(F) \cdot \sqrt{t d_{S}} \in \mathrm{H}^{*}(S, \mathbb{Z})
$$

и назовем его вектором, ассоциированным с $F$ (или вектором Мукаи).

На решетке когомологий $\mathrm{H}^{*}(S, \mathbb{Z})$ определим симметрическую билинейную форму по правилу

$$
\left(u, u^{\prime}\right)=r \cdot s^{\prime}+s \cdot r^{\prime}-\alpha \cdot \alpha^{\prime} \in \mathrm{H}^{4}(S, \mathbb{Z}) \cong \mathbb{Z}
$$

для всякой пары $u=(r, \alpha, s), u^{\prime}=\left(r^{\prime}, \alpha^{\prime}, s^{\prime}\right) \in \mathrm{H}^{0}(S, \mathbb{Z}) \oplus \mathrm{H}^{2}(S, \mathbb{Z}) \oplus \mathrm{H}^{4}(S, \mathbb{Z})$. Решетку когомологий $\mathrm{H}^{*}(S, \mathbb{Z})$ с этой билинейной формой $(\cdot, \cdot)$ назовем решеткой Мукаи и обозначим через $\widetilde{\mathrm{H}}(S, \mathbb{Z})$. Отметим, что данная билинейная форма отличается от обычной формы пересечения знаком $(-)$ на вторых когомологиях. И, значит, решетка Мукаи $\widetilde{\mathrm{H}}(S, \mathbb{Z})$ изоморфна решетке $\mathrm{U} \perp-\mathrm{H}^{2}(S, \mathbb{Z})$, где $\mathrm{U}$ - гиперболическая решетка $\left(\begin{array}{ll}0 & 1 \\ 1 & 0\end{array}\right)$.

Для любых двух объектов $F$ и $G$ спаривание $(v(F), v(G))$ есть по определению $\mathrm{H}^{4}$-компонента элемента $c h(F)^{\vee} \cdot c h(G) \cdot t d_{S}$. Следовательно, по теореме РиманаРоха-Гротендика имеем равенство:

$$
(v(F), v(G))=\chi(F, G):=\sum_{i}(-1)^{i} \operatorname{dim} \operatorname{Ext}^{i}(F, G) .
$$

На решетках $\widetilde{\mathrm{H}}(S, \mathbb{Z})$ и $\mathrm{T}_{S}$ имеются естественные структуры Ходжа. В данном случае под структурами Ходжа понимается то, что в пространствах $\widetilde{\mathrm{H}}(S, \mathbb{C})$ и $\mathrm{T}_{S} \otimes \mathbb{C}$ зафииксировано одномерное подпространство $\mathrm{H}^{2,0}(S)$.

ОПРЕДЕЛЕНИЕ 4.1.1. Пусть $S_{1}$ и $S_{2}$ - две К3 поверхности. Скажем, что их решетки Мукаи (соответственно решетки трансцедентных циклов) ходжево изометричны, если существует изометрия между ними, которая переводит одномерное подпространство $\mathrm{H}^{2,0}\left(S_{1}\right)$ в $\mathrm{H}^{2,0}\left(S_{2}\right)$.

Пусть сейчас $\mathscr{E}$ - это произвольньй объект из производной категории произведения $\mathbf{D}^{b}\left(S_{1} \times S_{2}\right)$. Рассмотрим алгебраический цикл

$$
Z_{\mathscr{E}}:=p^{*} \sqrt{t d_{S_{1}}} \cdot \operatorname{ch}(\mathscr{E}) \cdot \pi^{*} \sqrt{t d_{S_{2}}}
$$

на произведении $S_{1} \times S_{2}$, где $p$ и $\pi-$ проекции в диаграмме

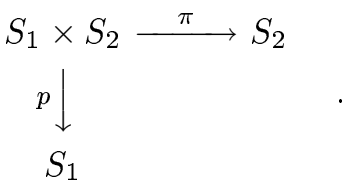

В случае К3 поверхностей цикл $Z_{\mathscr{E}}$, который a priori является рациональным, на самом деле целый. 
Лемма 4.1.2 [31]. Для всякого облекта $\mathscr{E} \in \mathbf{D}^{b}\left(S_{1} \times S_{2}\right)$ характер Черна сh(E) и иикл $Z_{\mathscr{E}}$ являются чельми, т.е. принадлежсат $\mathrm{H}^{*}\left(S_{1} \times S_{2}, \mathbb{Z}\right)$.

Таким образом, цикл $Z_{\mathscr{E}}$ задает отображение из решетки целых когомологий поверхности $S_{1}$ в целые когомологии $S_{2}$ :

$$
\begin{array}{ccc}
f_{Z_{\mathscr{E}}}: \mathrm{H}^{*}\left(S_{1}, \mathbb{Z}\right) & \longrightarrow & \mathrm{H}^{*}\left(S_{2}, \mathbb{Z}\right) \\
\cup & & \cup \\
\alpha & \mapsto & \pi_{*}\left(Z_{\mathscr{E}} \cdot p^{*}(\alpha)\right)
\end{array}
$$

Следующее предложение аналогично теореме 4.9 из работы [31].

ПРЕДЛОЖЕНИЕ 4.1.3. Если для облекта $\mathscr{E}$ функтор $\Phi_{\mathscr{E}}$ из категории $\mathbf{D}^{b}\left(S_{1}\right)$ в категорию $\mathbf{D}^{b}\left(S_{2}\right)$ является вполне строгим, тогда:

1) $f_{Z_{\mathscr{E}}}$ является изометрией межсду решетками $\widetilde{\mathrm{H}}\left(S_{1}, \mathbb{Z}\right)$ и $\widetilde{\mathrm{H}}\left(S_{2}, \mathbb{Z}\right)$,

2) обратное отображение $\kappa f$ совпадает с гомоморфизмом

$$
\begin{array}{ccc}
f^{\prime}: \quad \mathrm{H}^{*}\left(S_{2}, \mathbb{Z}\right) & \longrightarrow & \mathrm{H}^{*}\left(S_{1}, \mathbb{Z}\right) \\
\cup & & \cup \\
\beta & \mapsto & p_{*}\left(Z_{\mathscr{E}}^{\vee} \cdot \pi^{*}(\beta)\right)
\end{array}
$$

определенным ииклом

$$
Z_{\mathscr{E}}^{\vee}=p^{*} \sqrt{t d_{S_{1}}} \cdot \operatorname{ch}\left(\mathscr{E}^{\vee}\right) \cdot \pi^{*} \sqrt{t d_{S_{2}}}
$$

əде $\mathscr{E}^{\vee}:=\mathbf{R} \underline{\mathscr{H} o m}\left(\mathscr{E}, \mathscr{O}_{S_{1} \times S_{2}}\right)$.

ДокАЗАТЕЛЬство. Левьй и правый сопряженные функторы к $\Phi_{\mathscr{E}}$ изоморфны и задаются по формуле:

$$
\Phi_{\mathscr{E}}^{*}=\Phi_{\mathscr{E}}^{!}=\mathbf{R} p_{*}\left(\mathscr{E}^{\vee} \stackrel{\mathbf{L}}{\otimes} \pi^{*}(\cdot)\right)[2] .
$$

Так как $\Phi_{\mathscr{E}}$ вполне строгий, то композиция $\Phi_{\mathscr{E}}^{*} \circ \Phi_{\mathscr{E}}$ изоморфина тождественному функTopy $\operatorname{id}_{\mathbf{D}^{b}\left(S_{1}\right)}$.

Тождественньй функтор $\mathrm{id}_{\mathbf{D}^{b}\left(S_{1}\right)}$ задается структурным пучком $\mathscr{O}_{\Delta}$ диагонали $\Delta \subset$ $S_{1} \times S_{1}$.

Используя формулу проекции и теорему Римана-Роха-Гротендика, можно найти, что композиция $f^{\prime} \circ f$ представляется циклом $p_{1}^{*} \sqrt{t d_{S_{1}}} \cdot \operatorname{ch}\left(\mathscr{O}_{\Delta}\right) \cdot p_{2}^{*} \sqrt{t d_{S_{1}}}$, где $p_{1}, p_{2}-$ проекции $S_{1} \times S_{1}$ на сомножители. Опять по теореме Римана-Роха-Гротендика находим, что этот цикл есть цикл $\Delta$. Следовательно, композиция $f^{\prime} \circ f$ является тождественным отображением, и, значит, $f$ есть изоморфизм между $\mathrm{H}^{*}\left(S_{1}, \mathbb{Z}\right)$ и $\mathrm{H}^{*}\left(S_{2}, \mathbb{Z}\right)$, по той причине, что обе они являются свободными абелевыми группами одинакового ранга.

Обозначим через $\nu_{S}: S \longrightarrow$ Spec $\mathbb{C}$ структурньй морфизм $S$. Тогда спаривание $\left(\alpha, \alpha^{\prime}\right)$ на решетке $\widetilde{\mathrm{H}}(S, \mathbb{Z})$ можно записать как $\nu_{*}\left(\alpha^{\vee} \cdot \alpha^{\prime}\right)$. Из формулы проекции получаем:

$$
\begin{aligned}
(\alpha, f(\beta)) & =\nu_{S_{2}, *}\left(\alpha^{\vee} \cdot \pi_{*}\left(\pi^{*} \sqrt{t d_{S_{2}}} \cdot \operatorname{ch}(\mathscr{E}) \cdot p^{*} \sqrt{t d_{S_{1}}} \cdot p^{*}(\beta)\right)\right) \\
& =\nu_{S_{2}, *} \pi_{*}\left(\pi^{*}\left(\alpha^{\vee}\right) \cdot p^{*}(\beta) \cdot \operatorname{ch}(\mathscr{E}) \cdot \sqrt{t d_{S_{1} \times S_{2}}}\right) \\
& =\nu_{S_{1} \times S_{2}, *}\left(\pi^{*}\left(\alpha^{\vee}\right) \cdot p^{*}(\beta) \cdot \operatorname{ch}(\mathscr{E}) \cdot \sqrt{t d_{S_{1} \times S_{2}}}\right)
\end{aligned}
$$


для произвольных $\alpha \in \mathrm{H}^{*}\left(S_{2}, \mathbb{Z}\right), \beta \in \mathrm{H}^{*}\left(S_{1}, \mathbb{Z}\right)$. Аналогичным образом имеем:

$$
\left(\beta, f^{\prime}(\alpha)\right)=\nu_{S_{1} \times S_{2}, *}\left(p^{*}\left(\beta^{\vee}\right) \cdot \pi^{*}(\alpha) \cdot \operatorname{ch}(\mathscr{E})^{\vee} \cdot \sqrt{t d_{S_{1} \times S_{2}}}\right) .
$$

Следовательно, $(\alpha, f(\beta))=\left(f^{\prime}(\alpha), \beta\right)$. Так как $f^{\prime} \circ f$ - тождественное отображение, то

$$
\left(f(\alpha), f\left(\alpha^{\prime}\right)\right)=\left(f^{\prime} f(\alpha), \alpha^{\prime}\right)=\left(\alpha, \alpha^{\prime}\right) .
$$

И, значит, $f$ - это изометрия.

4.2. Критерий эквивалентности для производных категорий когерентных пучков. В этом разделе дается критерий того, когда производные категории когерентных пучков на двух К3 поверхностях эквивалентны как триангулированные категории. Этот критерий по виду очень напоминает строгую теорему Торелли для К3 поверхностей, которая говорит, что две К3 поверхности $S_{1}$ и $S_{2}$ изоморфны тогда и только тогда, когда их решетки вторых когомологий ходжево изометричны, т.е. существует изометрия

$$
\mathrm{H}^{2}\left(S_{1}, \mathbb{Z}\right) \stackrel{\sim}{\longrightarrow} \mathrm{H}^{2}\left(S_{2}, \mathbb{Z}\right),
$$

продолжение которой до отображения комплексных когомологий переводит подпространство $\mathrm{H}^{2,0}\left(S_{1}\right)$ в $\mathrm{H}^{2,0}\left(S_{2}\right)$ (см. [39], [27]).

Наш главньй результат этой главы вьглядит так.

Теорема 4.2.1. Пусть $S_{1}$ и $S_{2}$ - две гладкие проективные К3 поверхности над полем комплексных чисел $\mathbb{C}$. Тогда производные категории когерентных пучков $\mathbf{D}^{b}\left(S_{1}\right)$ и $\mathbf{D}^{b}\left(S_{2}\right)$ әквивалентны как триангулированные категории тогда и только тогда, когда существует ходжева изометрия $f: \widetilde{\mathrm{H}}\left(S_{1}, \mathbb{Z}\right) \stackrel{\sim}{\longrightarrow} \widetilde{\mathrm{H}}\left(S_{2}, \mathbb{Z}\right)$ между решетками Мукаи поверхностей $S_{1}$ u $S_{2}$.

Эта теорема имеет и другой вариант, которьй также может быть интересен (см. теорему 4.2.4).

Доказательство теоремы 4.2.1 разобьем на два предложения. Доказательство первого предложения существенно опирается на главную теорему 3.2.1 предыдущей главы, так как использует тот факт, что любая эквивалентность может быть представлена объектом на произведении.

ПРЕДЛОЖЕНИЕ 4.2.2. Пусть $S_{1}$ u $S_{2}-\partial в е$ K3 поверхности такие, что их производные категории когерентных пучков әквивалентны. Тогда существует ходжева изометрия между решетками трансцедентных ииклов $\mathrm{T}_{S_{1}}$ u $\mathrm{T}_{S_{2}}$.

ДокАЗАТЕЛЬство. По теореме 3.2 .2 существует объект $\mathscr{E}$ на произведении $S_{1} \times$ $S_{2}$, которьй задает эквивалентность. Из предложения 4.1 .3 следует, что $f_{Z_{\mathscr{E}}}$ задает ходжеву изометрию между решетками Мукаи $\widetilde{\mathrm{H}}\left(S_{1}, \mathbb{Z}\right)$ и $\widetilde{\mathrm{H}}\left(S_{2}, \mathbb{Z}\right)$. Так как цикл $Z$ является алгебраическим, получаем две изометрии $f_{\text {alg }}:-\mathrm{NS}\left(S_{1}\right) \perp \mathrm{U} \stackrel{\sim}{\longrightarrow}-\mathrm{NS}\left(S_{2}\right) \perp \mathrm{U}$ и $f_{\tau}: \mathrm{T}_{S_{1}} \stackrel{\sim}{\longrightarrow} \mathrm{T}_{S_{2}}$, где $\mathrm{NS}\left(S_{1}\right), \mathrm{NS}\left(S_{2}\right)$ - решетки Нерона-Севери и $\mathrm{T}_{S_{1}}, \mathrm{~T}_{S_{2}}-$ решетки трансцедентных циклов. Очевидно, что $f_{\tau}$ является ходжевой изометрией.

Доказательство в обратную сторону существенно использует результаты статьи [31], где исследованы многообразия модулей расслоений на К3 поверхностях, а также использует теорему 2.1.5, в которой дается критерий того, когда функтор является вполне строгим (см. [7]). 
ПРЕДЛОЖЕНИЕ 4.2.3. Пусть $S_{1} u S_{2}$ - две проективные К3 поверхности. Предположим, что существует ходжева изометрия

$$
f: \widetilde{\mathbf{H}}\left(S_{2}, \mathbb{Z}\right) \stackrel{\sim}{\longrightarrow} \widetilde{\mathbf{H}}\left(S_{1}, \mathbb{Z}\right) .
$$

Тогда ограниченные производные категории когерентных пучков $\mathbf{D}^{b}\left(S_{1}\right)$ u $\mathbf{D}^{b}\left(S_{2}\right)$ эквивалентны.

ДоКАЗАТЕЛЬСТВо. Положим $v=f(0,0,1)=(r, l, s)$ и $u=f(1,0,0)=(p, k, q)$. Без потери общности можно предполагать, что $r>1$. Действительно, на решетке Мукаи сушествуют два типа ходжевых изометрий. Первьй тип - это умножение на характер Черна $e^{m}$ линейного расслоения:

$$
\varphi_{m}(r, l, s)=\left(r, l+r m, s+(m, l)+\frac{r}{2} m^{2}\right)
$$

Второй тип - это перестановка $r$ и $s$. Используя эти два типа перестановок, можно заменить $f$ таким образом, чтобы $r$ стало больше 1 .

Очевидно, что вектор $v \in \mathrm{U} \perp-\mathrm{NS}\left(S_{1}\right)$ является изотропным, т.е. $(v, v)=0$. В замечательной работе Мукаи [31] доказано, что в этом случае существует поляризация $A$ на поверхности К $3 S_{1}$ такая, что многообразие модулей $\mathscr{M}_{A}(v)$ расслоений, стабильных относительно поляризации $A$, для которых вектор Мукаи совпадает с $v$, является гладкой проективной К3 поверхностью. Более того, так как существует вектор $u \in \mathrm{U} \perp-\mathrm{NS}\left(S_{1}\right)$ такой, что $(v, u)=1$, то многообразие модулей $\mathscr{M}_{A}(v)$ является тонким. Следовательно, существует универсальное расслоение $\mathscr{E}$ на произведении $S_{1} \times \mathscr{M}_{A}(v)$.

Универсальное расслоение $\mathscr{E}$ задает функтор $\Phi_{\mathscr{E}}: \mathbf{D}^{b}\left(\mathscr{M}_{A}(v)\right) \longrightarrow \mathbf{D}^{b}\left(S_{1}\right)$. Для этого функтора условия теоремы 2.1.5 легко проверяются. Действительно $\Phi_{\mathscr{E}}\left(\mathscr{O}_{t}\right)=$ $\mathscr{E}_{t}$, где $\mathscr{E}_{t}-$ стабильное расслоение на $S_{1}$, для которого $v\left(\mathscr{E}_{t}\right)=v$. Все эти пучки простые, и, конечно, $\operatorname{Ext}^{i}\left(\mathscr{E}_{t}, \mathscr{E}_{t}\right)=0$ для $i \notin[0,2]$. Что дает условие 2) теоремы 2.1.5.

Так как $E_{t}$ стабильны, то $\operatorname{Hom}\left(\mathscr{E}_{t_{1}}, \mathscr{E}_{t_{2}}\right)=0$. И по двойственности Серра $\operatorname{Ext}^{2}\left(\mathscr{E}_{t_{1}}, \mathscr{E}_{t_{2}}\right)=0$. Изотропность вектора $v$ влечет, что и $\operatorname{Ext}^{1}\left(\mathscr{E}_{t_{1}}, \mathscr{E}_{t_{2}}\right)=0$. Таким образом, пучки $\mathscr{E}_{t_{1}}$ и $\mathscr{E}_{t_{2}}$ ортогональны для любых разных точек $t_{1}, t_{2}$. По теореме 2.1.5 получаем, что функтор $\Phi_{\mathscr{E}}$ является вполне строгим.

На самом деле, функтор $\Phi_{\mathscr{E}}$ не только вполне строгий, но и является эквивалентностью. Следуюшее рассуждение, основанное на доказательстве теоремы 3.3 из [9] показывает это. Обозначим через $\mathscr{D}$ образ категории $\mathbf{D}^{b}\left(\mathscr{M}_{A}(v)\right)$ в $\mathbf{D}^{b}\left(S_{1}\right)$. Так как она является допустимой (см. определение 2.2.2), то существуют правый и левый ортогоналы к ней, которые из-за того, что канонический класс на К3 поверхности тривиален, совпадают друг с другом в данном случае. Таким образом, полуортогональное разложение вида $\left\langle\mathscr{D}^{\perp}, \mathscr{D}\right\rangle$ является полностью ортогональным. Рассмотрим очень обильное линейное расслоение $\mathscr{L}$ на $\mathscr{M}_{A}(v)$. Все степени $\mathscr{L}^{i}$ являются неразложимыми объектами и, значит, принадлежат какой-нибудь подкатегории $\mathscr{D}$ или $\mathscr{D}^{\perp}$, и все - одной и той же, так как никакая пара из них не является полностью ортогональной. Но $\left\{\mathscr{L}^{i}\right\}$ образуют обильную последовательность (см. определение 3.4.1). По лемме 3.4.3 ортогонал к подкатегории, порожденной обильной последовательностью, равен 0. Таким образом, так как $\mathscr{D}$ не тривиальна, получаем, что $\mathscr{D}^{\perp}=0$. Следовательно, $\Phi_{\mathscr{E}}-$ эквивалентность. 
Далее, цикл $Z_{\mathscr{E}}$, определенньй по формуле (47), индуцирует ходжеву изометрию $g: \widetilde{\mathrm{H}}\left(\mathscr{M}_{A}(v), \mathbb{Z}\right) \longrightarrow \widetilde{\mathrm{H}}\left(S_{1}, \mathbb{Z}\right)$ такую, что $g(0,0,1)=v=(r, l, s)$. Следовательно, $f^{-1} \circ g$ также является ходжевой изометрией и переводит $(0,0,1)$ в $(0,0,1)$. Следовательно, $f^{-1} \cdot g$ индуцирует ходжеву изометрию между решетками вторых когомологий поверхностей $S_{2}$ и $\mathscr{M}_{A}(v)$. И, следовательно, по теореме Торелли поверхности $S_{2}$ и $\mathscr{M}_{A}(v)$ изоморфны [39], [27].

Данное предложение и предложение 4.1.3 дают доказательство теоремы 4.2.1. Существует другая версия теоремы 4.2.1, которая дает критерий эквивалентности производных категорий в терминах решетки трансцедентных циклов.

Теорема 4.2.4. Пусть $S_{1}$ и $S_{2}$ - две гладкие проективные К3 поверхности над полем $\mathbb{C}$. Тогда производные категории когерентных пучков $\mathbf{D}^{b}\left(S_{1}\right)$ u $\mathbf{D}^{b}\left(S_{2}\right)$ әквивалентны как триангулированные категории тогда и только тогда, когда существует ходжева изометрия $f_{\tau}: \mathrm{T}_{S_{1}} \stackrel{\sim}{\longrightarrow} \mathrm{T}_{S_{2}}$ между решетками трансцедентных ииклов поверхностей $S_{1}$ и $S_{2}$.

Это утверждение является следствием теоремы 4.2.1 и следуюшего предложения.

ПРЕДЛОЖЕНИЕ 4.2 .5 [33]. Пусть $\varphi_{1}, \varphi_{2}: \mathrm{T} \longrightarrow \mathrm{H}-$ два примитивных вложения решетки Т в четную унимодулярную решетку Н. Предположим, что ортогональное дополнение $\mathrm{N}:=\varphi_{1}(\mathrm{~T})^{\perp}$ в $\mathrm{H}$ содержит гиперболическую решетку $\mathrm{U}=\left(\begin{array}{ll}0 & 1 \\ 1 & 0\end{array}\right)$ как подрешетку. Тогда $\varphi_{1}$ и $\varphi_{2}$ әквивалентны, т.е. существует изометрия $\gamma$ решетки $\mathrm{H}$ такая, что $\varphi_{1}=\gamma \varphi_{2}$.

Действительно, предположим, что имеется ходжева изометрия

$$
f_{\tau}: \mathrm{T}_{S_{2}} \stackrel{\sim}{\longrightarrow} \mathrm{T}_{S_{1}}
$$

Мы знаем, что ортогональное дополнение к решетке трансцедентных циклов $\mathrm{T}_{S}$ в решетке Мукаи $\widetilde{\mathrm{H}}(S, \mathbb{Z})$ изоморфно решетке - $\mathrm{NS}(S) \perp \mathrm{U}$. И, значит, по предыдушему предложению 4.2.5 существует изометрия

$$
f: \widetilde{\mathrm{H}}\left(S_{2}, \mathbb{Z}\right) \stackrel{\sim}{\longrightarrow} \widetilde{\mathrm{H}}\left(S_{1}, \mathbb{Z}\right)
$$

такая, что $\left.f\right|_{\mathrm{T}_{S_{2}}}=f_{\tau}$. Таким образом, изометрия $f$ является также ходжевой. Следовательно, по теореме 4.2.1 производные категории когерентных пучков на поверхностях $S_{1}$ и $S_{2}$ эквивалентны.

\section{Глава 5. Абелевы многообразия}

5.1. Эквивалентности между категориями когерентных пучков на абелевых многообразиях. В этой главе изучаются производные категории когерентных пучков на абелевых многообразиях и их групшы автоэквивалентностей. Пусть $A$ - абелево многообразие размерности $n$ на полем $k$. Будем обозначать через $m: A \times A \rightarrow A$ морфизм композиции, которьй считается определенным над полем $k$, и через $e-k$-точку, которая является единищей групповой структуры. Для любой $k$-точки $a \in A$ сушествует автоморфизм сдвига $m(\cdot, a): A \rightarrow A$, который будет обозначаться $T_{a}$. 
Обозначим через $\widehat{A}$ двойственное абелево многообразие, которое является многообразием модулей линейных расслоений на $A$, принадлежаших $\operatorname{Pic}^{0}(A)$. Более того, $\widehat{A}$ является тонким многообразием модулей. Поэтому на произведении $A \times \widehat{A}$ существует универсальное линейное расслоение $\mathscr{P}$, которое называется расслоением Пуанкаре. Это расслоение определяется однозначно тем, что для любой $k$-точки $\alpha \in \widehat{A}$ ограничение $\mathscr{P}$ на $A \times\{\alpha\}$ изоморфно линейному расслоению из $\operatorname{Pic}^{0}(A)$, соответствуюшему $\alpha$, и, в дополнение, ограничение $\left.\mathscr{P}\right|_{\{e\} \times \widehat{A}}$ должно быть тривиальным.

ОПРЕДЕЛЕНИЕ 5.1. Линейное расслоение на $A$, которое соответствует $k$-точке $\alpha \in \widehat{A}$, будем обозначать далее $\mathscr{P}_{\alpha}$.

Более того, если имеется несколько абелевых многообразий $A_{1}, \ldots, A_{m} u$ $k$-точка $\left(\alpha_{1}, \ldots, \alpha_{m}\right) \in \widehat{A}_{1} \times \cdots \times \widehat{A}_{m}$, то будем обозначать через $\mathscr{P}_{\left(\alpha_{1}, \ldots, \alpha_{k}\right)}$ линейное расслоение $\mathscr{P}_{\alpha_{1}} \bigotimes \cdots \otimes \mathscr{P}_{\alpha_{k}}$ на произведении $A_{1} \times \cdots \times A_{k}$.

Для любого гомоморфизма абелевых многообразий $f: A \rightarrow B$ определен двойственный гомоморфизм $\widehat{f}: \widehat{B} \rightarrow \widehat{A}$. Поточечно он устроен так, что точку $\beta \in \widehat{B}$ переводит в точку $\alpha \in \widehat{A}$ тогда и только тогда, когда линейное расслоение $f^{*} \mathscr{P} \beta$ совпадает с расслоением $\mathscr{P} \alpha$ на $A$.

Дважды двойственное абелево многообразие $\widehat{\widehat{A}}$ может быть естественным образом отождествлено с $A$ при помощи расслоений Пуанкаре на $A \times \widehat{A}$ и на $\widehat{A} \times \widehat{\widehat{A}}$. Другими словами, сушествует единственный изоморфизм $\kappa_{A}: A \stackrel{\sim}{\rightarrow} \widehat{\widehat{A}}$ такой, что поднятие расслоения Пуанкаре $\mathscr{P} \widehat{A}$ при изоморфизме $1 \times \kappa_{A}: \widehat{A} \times A \stackrel{\sim}{\longrightarrow} \widehat{A} \times \widehat{A}$ совпадает с расслоением Пуанкаре $\mathscr{P}_{A}$, т.е. $\left(1 \times \kappa_{A}\right)^{*} P_{\widehat{A}} \cong \mathscr{P}_{A}$.

Таким образом, ^ является инволюцией на категории абелевых многообразий, т.е. контравариантным функтором, чей квадрат изоморфен тождественному функтору $\kappa: \mathrm{id} \stackrel{\sim}{\sim}$.

Расслоение Пуанкаре $\mathscr{P}$ предоставляет нам пример точной эквивалентности между производными категориями когерентных пучков двух, в обшем случае неизоморфных, многообразий $A$ и $\widehat{A}$. Рассмотрим проекции

$$
A \stackrel{p}{\longleftarrow} A \times \widehat{A} \stackrel{q}{\longrightarrow} \widehat{A}
$$

и функтор $\Phi_{\mathscr{P}}: \mathbf{D}^{b}(A) \longrightarrow \mathbf{D}^{b}(\widehat{A})$, определенный по формуле $(7)$ :

$$
\Phi_{\mathscr{P}}(\cdot)=\mathbf{R} q_{*}\left(\mathscr{P} \otimes p^{*}(\cdot)\right) .
$$

Следующее предложение доказано в [29].

ПРЕДЛОЖЕНИЕ 5.1 .2 [29]. Пусть Я્Р-расслоение Пуанкаре на $A \times \widehat{A}$. Тогда функтор $\Phi_{\mathscr{P}}: \mathbf{D}^{b}(A) \longrightarrow \mathbf{D}^{b}(\widehat{A})$ является точной әквивалентностью, и существует изоморфизм функторов

$$
\Psi_{\mathscr{P}} \circ \Phi_{\mathscr{P}} \cong\left(-1_{A}\right)^{*}[n],
$$

где $\left(-1_{A}\right)$ - отображсение взятия обратного. 
ЗАмЕчАниЕ 5.1.3. В статье [29] это утверждение доказано для абелевых многообразий над алгебраически замкнутьм полем. Однако оно верно и над произвольным полем, так как двойственное многообразие и расслоение Пуанкаре определены всегда над тем же самьм полем (см., например, [32]). А утверждение про эквивалентность будет следовать из леммы 5.1.9.

Рассмотрим $k$-точку $(a, \alpha) \in A \times \widehat{A}$. С каждой такой точкой можно связать функтор из $\mathbf{D}^{b}(A)$ в себя по правилу:

$$
\Phi_{(a, \alpha)}(\cdot):=T_{a *}(\cdot) \otimes \mathscr{P}_{\alpha}=T_{-a}^{*}(\cdot) \otimes \mathscr{P}_{\alpha} \cdot
$$

Функтор $\Phi_{(a, \alpha)}$ представляется пучком

$$
S_{(a, \alpha)}=\mathscr{O}_{\Gamma_{a}} \otimes p_{2}^{*}\left(\mathscr{P}_{\alpha}\right)
$$

на произведении $A \times A$, где $\Gamma_{a}$ - это график автоморфизма сдвига $T_{a}$. Очевидно, что функтор $\Phi_{(a, \alpha)}$ является автоэквивалентностью.

Множество функторов $\Phi_{(a, \alpha)}$, параметризованных $A \times \widehat{A}$, можно соединить в один функтор из $\mathbf{D}^{b}(A \times \widehat{A})$ в $\mathbf{D}^{b}(A \times A)$, которьй будет переводить структурньй пучок точки $\mathscr{O}_{(a, \alpha)}$ в $S_{(a, \alpha)}$. (Заметим, что этим условием функтор определяется не однозначно, а только с точностью до умножения на линейное расслоение, поднятое с $A \times \widehat{A}$.)

Мы определим этот функтор $\Phi_{S_{A}}: \mathbf{D}^{b}(A \times \widehat{A}) \longrightarrow \mathbf{D}^{b}(A \times A)$ как композицию двух других.

Рассмотрим объект $\mathrm{P}_{A}=p_{14}^{*} \mathscr{O}_{\Delta} \otimes p_{23}^{*} \mathscr{P} \in \mathbf{D}^{b}((A \times \widehat{A}) \times(A \times A))$ и обозначим через $\mu_{A}: A \times A \longrightarrow A \times A$ морфизм, который переводит точку $\left(a_{1}, a_{2}\right)$ в $\left(a_{1}, m\left(a_{1}, a_{2}\right)\right)$. У нас появляются два функтора:

$$
\Phi_{\mathrm{P}_{A}}: \mathbf{D}^{b}(A \times \widehat{A}) \longrightarrow \mathbf{D}^{b}(A \times A), \quad \mathbf{R} \mu_{A *}: \mathbf{D}^{b}(A \times A) \longrightarrow \mathbf{D}^{b}(A \times A) .
$$

ОПРЕДЕЛЕНИЕ 5.1.4. Функтор $\Phi_{S_{A}}$ есть композищия $\mathbf{R} \mu_{A *} \circ \Phi_{\mathrm{P}_{A}}$.

ПРЕДЛОЖЕНИЕ 5.1.5. Функтор $\Phi_{S_{A}}$ является әквивалентностью и для каждой $k$-точки $(a, \alpha) \in A \times \widehat{A}$

а) структурный пучок точки $\mathscr{O}_{(a, \alpha)}$ переводит в пучок $S_{(a, \alpha)}$, определенныи формулой (50);

b) линейное расслоение $\mathscr{P}_{(\alpha, a)}$ на $A \times \widehat{A}$ переводит в обгект $\mathscr{O}_{\{-a\} \times A} \otimes$ $p_{2}^{*} \mathscr{P}_{\alpha}[n]$.

ДоКАЗАТЕльСТВо. Функтор $\Phi_{S_{A}}$ яВляется по определению композицией функторов $\mathbf{R} \mu_{A *}$ и $\Phi_{\mathrm{P}_{A}}$, которые суть эквивалентности: первьй по очевидным соображениям, а для второго это следует из предложений 2.1.7 и 5.1.2.

Функтор $\Phi_{\mathrm{P}_{A}}$ переводит структурньй пучок точки $\mathscr{O}_{(a, \alpha)}$ в пучок $\mathscr{O}_{A \times\{a\}} \otimes p_{1}^{*} \mathscr{P}_{\alpha}$. Далее функтор $\mathbf{R} \mu_{A *}$ посылает пучок $\mathscr{O}_{A \times\{a\}} \otimes p_{1}^{*} \mathscr{P}_{\alpha}$ в пучок $\mathscr{O}_{\Gamma_{a}} \otimes p_{1}^{*}\left(\mathscr{P}_{\alpha}\right)$.

Таким же образом, применяя предложение 5.1 .2 , находим, что функтор $\Phi_{\mathrm{P}_{A}}$ переводит линейное расслоение $\mathscr{P}_{(\alpha, a)}$ в объект $\mathscr{O}_{\{-a\} \times A} \otimes p_{2}^{*} \mathscr{P}_{\alpha}[n]$, а функтор $\mathbf{R} \mu_{A *}$ посылает объект $\mathscr{O}_{\{-a\} \times A} \otimes p_{2}^{*} \mathscr{P}_{\alpha}[n]$ в себя.

Предположим, что $A$ и $B$ - два абелевых многообразия, производные категории когерентных пучков которых эквивалентны. Зафиксируем некоторую эквивалентность. 
По теореме 3.2.2 она представляется объектом на произведении. Таким образом, имеется объект $\mathscr{E} \in \mathbf{D}^{b}(A \times B)$ и эквивалентность $\Phi_{\mathscr{E}}: \mathbf{D}^{b}(A) \stackrel{\sim}{\longrightarrow} \mathbf{D}^{b}(B)$.

Рассмотрим функтор

$$
A d_{\mathscr{E}}: \mathbf{D}^{b}(A \times A) \stackrel{\sim}{\longrightarrow} \mathbf{D}^{b}(B \times B),
$$

которьй определяется формулой (11) и является эквивалентностью. Рассмотрим композицию функторов $\Phi_{S_{B}}^{-1} \circ A d_{\mathscr{E}} \circ \Phi_{S_{A}}$.

ОПРЕДЕЛЕНИЕ 5.1.6. Обозначим через $\mathscr{J}(\mathscr{E})$ объект, представляюший функтор

$$
\Phi_{S_{B}}^{-1} \circ A d_{\mathscr{E}} \circ \Phi_{S_{A}} .
$$

Таким образом, сушествует коммутативная диаграмма:

$$
\begin{gathered}
\mathbf{D}^{b}(A \times \widehat{A}) \stackrel{\Phi_{S_{A}}}{\longrightarrow} \mathbf{D}^{b}(A \times A) \\
\Phi_{\mathscr{J}(\mathscr{E})} \downarrow \\
\mathbf{D}^{b}(B \times \widehat{B}) \stackrel{A_{\mathscr{E}}}{\stackrel{\Phi_{S_{B}}}{\longrightarrow}} \mathbf{D}^{b}(B \times B)
\end{gathered} .
$$

Следующая теорема позволяет вычислить объект $\mathscr{J}(\mathscr{E})$ и является основной при описании абелевых многообразий, обладающих эквивалентными производными категориями когерентных пучков.

ТЕОРема 5.1.7. Существуют гомоморфизм абелевых многообразий $f_{\mathscr{E}}: A \times \widehat{A}$ $\longrightarrow B \times \widehat{B}$, являюшийся изоморфизмом, и линейное расслоение $\mathscr{L}_{\mathscr{E}}$ на $A \times \widehat{A}$ такое, что облект $\mathscr{J}(\mathscr{E})$ изоморфен $i_{*}\left(\mathscr{L}_{\mathscr{E}}\right)$, где $i$ - это вложсение многообразия $A \times \widehat{A}$ в $(A \times \widehat{A}) \times(B \times \widehat{B})$ в качестве графика изоморфизма $f_{\mathscr{E}}$.

Прежде чем перейти к доказательству теоремы, сформулируем две леммы, которые позволят нам считать поле $k$ алгебраически замкнутым. Обозначим через $\bar{k}$ алгебраическое замыкание $k$. Положим $\bar{X}:=X \times_{\operatorname{Spec}(k)} \operatorname{Spec}(\bar{k})$ и обозначим через $\mathscr{F}$ обратный образ $\mathscr{F}$ относительно морфизма $\bar{X} \longrightarrow X$.

ЛЕмма 5.1.8 [37]. Пусть $\mathscr{F}$ - когерентный пучок на гладком многообразии X. Предположим, что существуют замкнутое подмногообразие $j: Z \hookrightarrow \bar{X}$ и обратимый пучок $\mathscr{L}$ на $Z$ такие, что $\overline{\mathscr{F}} \cong j_{*} \mathscr{L}$. Тогда существуют замкнутое подмногообразие $i: Y \hookrightarrow X$ и обратимый пучок $\mathscr{M}$ на $Y$ такие, что $\mathscr{F} \cong i_{*} \mathscr{M} u$ $j=\bar{i}$.

Следуюшая лемма говорит нам, что свойство функтора быть вполне строгим (эквивалентностью) стабильно относительно расширения полей.

ЛЕмма 5.1.9 [37]. Пусть $X$ и $Y$ - гладкие проективные многообразия над полем $k$, и пусть $\mathscr{E}$ - оббект производной категории $\mathbf{D}^{b}(X \times Y)$. Рассмотрим расширение полей $F / k$ и многообразия

$$
X^{\prime}=X \times_{\operatorname{Spec}(k)} \operatorname{Spec}(F), \quad Y^{\prime}=Y \times_{\operatorname{Spec}(k)} \operatorname{Spec}(F) .
$$

Обозначим через $\mathscr{E}^{\prime}$ поднятие облекта $\mathscr{E}$ в категорию $\mathbf{D}^{b}\left(X^{\prime} \times Y^{\prime}\right)$. Тогда функтор $\Phi_{\mathscr{E}}: \mathbf{D}^{b}(X) \longrightarrow \mathbf{D}^{b}(Y)$ вполне строгий (эквивалентность), если и только если функтор $\Phi_{\mathscr{E}^{\prime}}: \mathbf{D}^{b}\left(X^{\prime}\right) \longrightarrow \mathbf{D}^{b}\left(Y^{\prime}\right)$ является вполне строгим (әквивалентностью). 
ДОКАЗАТЕЛЬСТВО ТЕОРЕМЫ 5.1.7. ПольЗуясь леммами 5.1.8 и 5.1.9, можно перейти к алгебраическому замыканию поля $k$.

Шаг 1. Обозначим через $e$ и $e^{\prime}$ замкнутые точки многообразий $A \times \widehat{A}$ и, соответственно, $B \times \widehat{B}$, которые являются единицами групповых структур. Рассмотрим пучок небоскребов $\mathscr{O}_{e}$ и вычислим его образ относительно функтора $\Phi \mathscr{f}(\mathscr{E})$. По определению

$$
\Phi_{\mathscr{J}(\mathscr{E})}=\Phi_{S_{B}}^{-1} \circ A d_{\mathscr{E}} \circ \Phi_{S_{A}} .
$$

По предложению 5.1 .5 функтор $\Phi_{S_{A}}$ переводит пучок $\mathscr{O}_{e}$ в структурньй пучок диагонали $\mathscr{O}_{\Delta(A)}$ на $A \times A$. Так как структурньй пучок диагонали представляет тождественньй функтор, то из формулы (12) следует, что $A d_{\mathscr{E}}\left(\mathscr{O}_{\Delta(A)}\right)$ есть структурный пучок диагонали $\mathscr{O}_{\Delta(B)}$ на многообразии $B \times B$. А он, в свою очередь, переходит в

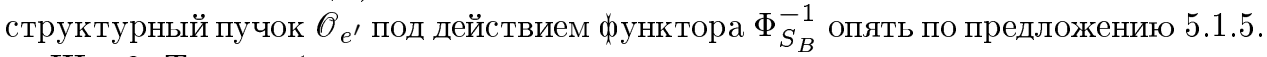

Шаг 2. Таким образом, получаем, что

$$
\mathscr{J}(\mathscr{E}) \stackrel{\mathrm{L}}{\otimes} \mathscr{O}_{\{e\} \times(B \times \widehat{B})} \cong \mathscr{O}_{\{e\} \times\left\{e^{\prime}\right\}} .
$$

Из этого следует, что существует аффинная окрестность $U=\operatorname{Spec}(R)$ точки $e$ в топологии Зариского такая, что объект $\mathscr{J}^{\prime}:=\left.\mathscr{J}(\mathscr{E})\right|_{U \times(B \times \widehat{B})}$ есть когерентньй пучок с носителем, который пересекает слой $\{e\} \times(B \times \widehat{B})$ в точке $\{e\} \times\left\{e^{\prime}\right\}$. Напомним, что носитель любого когерентного пучка является замкнутым подмножеством.

Рассмотрим теперь некоторую аффинную окрестность $V=\operatorname{Spec}(S)$ точки $e^{\prime}$ в $B \times \widehat{B}$. Пересечение носителя пучка $g^{\prime}$ с дополнением $B \times \widehat{B} \backslash V$ является замкнутым подмножеством, проекция которого на $A \times \widehat{A}$ - замкнутое подмножество, не содержащее точку $e$.

Таким образом, уменьшая, если необходимо, $U$, можно считать что оно все еще аффинно, а носитель пучка $\mathscr{J}^{\prime}$ содержится в $U \times V$. Это значит, что существует когерентный пучок $\mathscr{F}$ на $U \times V$ такой, что $j_{*}(\mathscr{F})=\mathscr{g}^{\prime}$, где $j$ - это вложение $U \times V$ в $U \times(B \times \widehat{B})$. Обозначим через $M$ конечно порожденный $R \otimes S$-модуль, который соответствует пучку $\mathscr{F}$, т.е. $\mathscr{F}=\widetilde{M}$. Кроме того, заметим, что $M$ является конечнопорожденным $R$-модулем, так как прямой образ при проекции когерентного пучка $\mathscr{J}^{\prime}=j_{*} \mathscr{F}$ является когерентным пучком.

Пусть $m$ - максимальный идеал в $R$, соответствуюший точке $e$. Мы знаем, что

$$
M \otimes_{R} R / m \cong R / m .
$$

Значит, сушествует гомоморфизм $R$-модулей $\varphi: R \longrightarrow M$, который после тензорного умножения на $R / m$ становится изоморфизмом. Таким образом, носители когерентных пучков $\operatorname{Ker} \varphi$ и $\operatorname{Coker} \varphi$ не содержат точку $e$. Поэтому, заменяя $U$ на меньшую аффинную окрестность точки $e$, которая не пересекается с носителями пучков $\operatorname{Ker} \varphi$ и Coker $\varphi$, мы получаем, что $\varphi$ есть изоморфизм. Следовательно, существует подсхема $X(U) \subset U \times(B \times \widehat{B})$ такая, что проекция $X(U) \longrightarrow U$ является изоморфизмом и

$$
\mathscr{J}^{\prime}=\left.\mathscr{J}(\mathscr{E})\right|_{U \times(B \times \widehat{B})} \cong \mathscr{O}_{X(U)} .
$$

Шаг 3. Мы получили тем самым, что для любой замкнутой точки $(a, \alpha) \in U$

$$
\Phi_{\mathscr{J}(\mathscr{E})}\left(\mathscr{O}_{(a, \alpha)}\right) \cong \mathscr{O}_{(b, \beta)}
$$


для некоторой замкнутой точки $(b, \beta) \in B \times \widehat{B}$. Если теперь рассмотреть произвольную замкнутую точку $(a, \alpha) \in A \times \widehat{A}$, то ее всегда можно представить как сумму $\left(a, a^{\prime}\right)=\left(a_{1}, \alpha_{1}\right)+\left(a_{2}, \alpha_{2}\right)$, где точки $\left(a_{1}, \alpha_{1}\right),\left(a_{2}, \alpha_{2}\right)$ принадлежат $U$. Обозначим через $\left(b_{1}, \beta_{1}\right)$ и $\left(b_{2}, \beta_{2}\right)$ образы этих точек относительно функтора $\Phi \mathscr{J}(\mathscr{E})$. Функтор $\Phi_{S_{A}}$, как мы знаем, переводит структурный пучок $\mathscr{O}_{(a, \alpha)}$ в пучок $S_{(a, \alpha)}$. Обозначим через $\mathscr{G}$ объект $A d_{\mathscr{E}}\left(S_{(a, \alpha)}\right)$. Чтобы вычислить его, воспользуемся соотношением (12). Имеется изоморфизм

$$
\Phi_{\mathscr{G}} \cong \Phi_{\mathscr{E}} \circ \Phi_{(a, \alpha)} \circ \Phi_{\mathscr{E}}^{-1}
$$

Но функтор $\Phi_{(a, \alpha)}$, которьй по определению $(49)$ есть $T_{a}^{*}(\cdot) \otimes \mathscr{P}_{\alpha}$, можно представить как композицию $\Phi_{\left(a_{1}, \alpha_{1}\right)} \Phi_{\left(a_{2}, \alpha_{2}\right)}$. Таким образом, получаем последовательность изоморфизмов

$$
\begin{aligned}
\Phi_{\mathscr{G}} & \cong \Phi_{\mathscr{E}} \circ \Phi_{(a, \alpha)} \circ \Phi_{\mathscr{E}}^{-1} \cong \Phi_{\mathscr{E}} \circ \Phi_{\left(a_{1}, \alpha_{1}\right)} \circ \Phi_{\mathscr{E}}^{-1} \\
& \cong \Phi_{\mathscr{E}} \circ \Phi_{\left(a_{2}, \alpha_{2}\right)} \circ \Phi_{\mathscr{E}}^{-1} \cong \Phi_{\left(b_{1}, \beta_{1}\right)} \circ \Phi_{\left(b_{2}, \beta_{2}\right)} \cong \Phi_{(b, \beta)}
\end{aligned}
$$

где $(b, \beta)=\left(b_{1}, \beta_{1}\right)+\left(b_{2}, \beta_{2}\right)$. И значит, объект $\mathscr{G}$ изоморфен $S_{(b, \beta)}$. Окончательно получаем, что

$$
\Phi_{\mathscr{J}(\mathscr{E})}\left(\mathscr{O}_{(a, \alpha)}\right) \cong \mathscr{O}_{(b, \beta)}
$$

для любой замкнутой точки $(a, \alpha) \in A \times \widehat{A}$.

Теперь, повторяя процедуру шага 2, можем для каждой замкнутой точки $\left(a, a^{\prime}\right)$ найти некоторую окрестность $W$ и подсхему $X(W) \subset W \times(B \times \widehat{B})$ такие, что проекция $X(W) \longrightarrow W$ является изоморфизмом, и $\left.\mathscr{J}\right|_{W \times(B \times \widehat{B})} \cong \mathscr{O}_{X(W)}$.

Склеивая все эти окрестности, находим подмногообразие $i: X \hookrightarrow(A \times \widehat{A}) \times(B \times \widehat{B})$ такое, что проекция $X \longrightarrow A \times \widehat{A}$ есть изоморфизм, а пучок $\mathscr{J}(\mathscr{E})$ изоморфен пучку $i_{*} \mathscr{L}$, где $\mathscr{L}$ - линейное расслоение на $X$. Подмногообразие $X$ задает гомоморфизм из $A \times \widehat{A}$ в $B \times \widehat{B}$, который индуцирует эквивалентность производных категорий. Следовательно, этот гомоморфизм является изоморфизмом.

В частности, из теоремы сразу следует, что если абелевы многообразия $A$ и $B$ имеют эквивалентные производные категории когерентных пучков, то многообразия $A \times \widehat{A}$ и $B \times \widehat{B}$ изоморфны. Ниже будет показано, что этот изоморфизм должен удовлетворять некоторому дополнительному условию (см. предложение 5.1.15).

СлЕДСТВИЕ 5.1.10. Изоморфизм $f_{\mathscr{E}}$ переводит $k$-точку $(a, \alpha) \in A \times \widehat{A}$ в точку $(b, \beta) \in B \times \widehat{B}$ тогда и только тогда, когда әквивалентности

$$
\Phi_{(a, \alpha)}: \mathbf{D}^{b}(A) \stackrel{\sim}{\longrightarrow} \mathbf{D}^{b}(A), \quad \Phi_{(b, \beta)}: \mathbf{D}^{b}(B) \stackrel{\sim}{\longrightarrow} \mathbf{D}^{b}(B)
$$

определенные по формуле (49), связаны следующим соотношением:

$$
\Phi_{(b, \beta)} \circ \Phi_{\mathscr{E}} \cong \Phi_{\mathscr{E}} \circ \Phi_{(a, \alpha)},
$$

или, в терминах обвектов,

$$
T_{b *} \mathscr{E} \otimes \mathscr{P}_{\beta} \cong T_{-a *} \mathscr{E} \otimes \mathscr{P}_{\alpha}=T_{a}^{*} \mathscr{E} \otimes \mathscr{P}_{\alpha}
$$


ДокАЗАТЕЛЬСтво. По теореме 5.1.7 функтор $\Phi_{\mathscr{J}(\mathscr{E})}$ переводит структурный пучок точки $\mathscr{O}_{(a, \alpha)}$ в структурный пучок точки $\mathscr{O}_{(b, \beta)}$, где $(b, \beta)=f_{\mathscr{E}}(a, \alpha)$. Из предложения 5.1.5 следует, что функтор $\Phi_{S_{A}}$ посылает пучок $\mathscr{O}_{(a, \alpha)}$ в $S_{(a, \alpha)}$. А пучок $S_{(a, \alpha)}$, в свою очередь, представляет функтор

$$
\Phi_{(a, \alpha)}=T_{a *}(\cdot) \otimes \mathscr{P}_{\alpha} .
$$

Теперь, используя диаграмму (51), находим, что при отображении $f_{\mathscr{E}}$ точка $(a, \alpha)$ переходит в точку $(b, \beta)$ тогда и только тогда, когда $S_{(b, \beta)} \cong A d_{\mathscr{E}}\left(S_{(a, \alpha)}\right)$. Применяя формулу (12), находим, что $\Phi_{(b, \beta)} \cong \Phi_{\mathscr{E}} \circ \Phi_{(a, \alpha)} \circ \Phi_{\mathscr{E}}^{-1}$.

В дальнейшем нам понадобится явная формула для объекта $\mathscr{J}(\mathscr{E})$ в частном случае, когда $A=B$ и эквивалентность $\Phi_{\mathscr{E}}$ есть $\Phi_{(a, \alpha)}$, определенная формулой (49).

ПреДЛОЖЕнИЕ 5.1.11. Пусть $A=B$. Рассмотрим облект $S_{(a, \alpha)}$ на $A \times A$, который представляет әквивалентность $\Phi_{(a, \alpha)}$, определенную формулой (49). Тогда пучок $\mathscr{J}\left(S_{(a, \alpha)}\right)$ есть $\Delta_{*} \mathscr{P}_{(\alpha,-a)}$, где $\Delta$ - это диагональное вложение $A \times \widehat{A} \boldsymbol{\theta}(A \times \widehat{A}) \times(A \times \widehat{A})$ и $\mathscr{P}_{(\alpha, a)}-$ линейное расслоение на $A \times \widehat{A}$, определенное e 5.1.1.

ДоКАЗАТЕльСТво. Из предложения 5.1.5 следует, что функтор $\Phi_{S_{A}}$ посылает структурный пучок $\mathscr{O}_{\left(a^{\prime}, \alpha^{\prime}\right)}$ в пучок $S_{\left(a^{\prime}, \alpha^{\prime}\right)}$ на $A \times A$ (формула (50)). Далее функтор $A d_{S_{(a, \alpha)}}$ переводит пучок $S_{\left(a^{\prime}, \alpha^{\prime}\right)}$ в себя, так как по формуле (12) объект $A d_{S_{(a, \alpha)}}\left(S_{\left(a^{\prime}, \alpha^{\prime}\right)}\right)$ представляет функтор

$$
\Phi_{(a, \alpha)} \circ \Phi_{\left(a^{\prime}, \alpha^{\prime}\right)} \circ \Phi_{(a, \alpha)}^{-1},
$$

который, в свою очередь, изоморфен $\Phi_{\left(a^{\prime}, \alpha^{\prime}\right)}$, так как все такие функторы коммутируют друг с другом. Таким образом, получаем, что функтор, задаваемьй пучком $\mathscr{J}\left(S_{(a, \alpha)}\right)$, переводит структурный пучок любой точки в себя, и, значит, это есть некоторое линейное расслоение $L$, сосредоточенное на диагонали.

Чтобы найти теперь линейное расслоение $L$, посмотрим, куда этот функтор переводит расслоение $\mathscr{P}_{\left(\alpha^{\prime}, a^{\prime}\right)}$. Снова применяя предложение 5.1.5, видим, что функтор

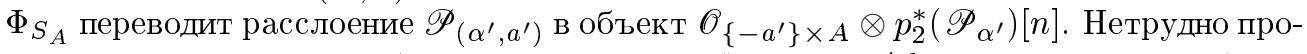
верить, что далее этот объект под действием функтора $A d_{S_{(a, \alpha)}}$ переходит в объект $\mathscr{O}_{\left\{-a^{\prime}+a\right\} \times A} \otimes p_{2}^{*}\left(\mathscr{P}_{\alpha^{\prime}+\alpha}\right)[n]$. И, следовательно, расслоение $\mathscr{P}_{\left(\alpha^{\prime}, a^{\prime}\right)}$ под действием функтора, задаваемого пучком $\mathscr{J}\left(S_{(a, \alpha)}\right)$, переходит в расслоение $\mathscr{P}_{\left(\alpha^{\prime}+\alpha, a^{\prime}-a\right)}$. То есть расслоение $L$ изоморфно $\mathscr{P}_{(\alpha,-a)}$.

Для абелевых многообразий $A$ и $B$ обозначим через $\mathrm{Eq}(A, B)$ множество всех точных эквивалентностей с точностью до изоморфизма из категории $\mathbf{D}^{b}(A)$ в категорию $\mathbf{D}^{b}(B)$.

Введем в рассмотрение два группоида $\mathfrak{A}$ и $\mathfrak{D}$ (т.е. категории, в которых все морфизмы обратимы). Объекты обоих - это абелевы многообразия. Морфизмами в группоиде $\mathfrak{A}$ будут изоморфизмы между ними как алгебраическими группами. Морфизмы в $\mathfrak{B}$ - это точные эквивалентности между категориями когерентных пучков на абелевых многообразиях, т.е.

$$
\begin{aligned}
\operatorname{Mor}_{\mathfrak{A}}(A, B) & :=\operatorname{Iso}(A, B), \\
\operatorname{Mor}_{\mathfrak{D}}(A, B) & :=\operatorname{Eq}(A, B) .
\end{aligned}
$$


По теореме 5.1.7 имеется отображение из множества $\operatorname{Eq}(A, B)$ в множество $\operatorname{Iso}(A \times \widehat{A}, B \times \widehat{B})$, которое сопоставляет эквивалентности $\Phi_{\mathscr{E}}$ изоморфизм $f_{\mathscr{E}}$. Рассмотрим отображение $F$ из $\mathfrak{D}$ в $\mathfrak{A}$, которое сопоставляет абелеву многообразию $A$ многообразие $A \times \widehat{A}$ и на морфизмах действует описанньм вьше способом.

ПРЕДЛОЖЕНИЕ 5.1.12. Отображсение $F: \mathfrak{D} \longrightarrow \mathfrak{A}$ является функтором.

ДокАЗАТЕльство. Чтобы доказать утверждение, надо только проверить, что $F$ уважает композицию морфизмов. Рассмотрим три абелевых многообразия $A, B, C$. И пусть $\mathscr{E}$ и $\mathscr{F}$ - объекты категорий $\mathbf{D}^{b}(A \times B)$ и $\mathbf{D}^{b}(B \times C)$ соответственно такие, что функторы

$$
\begin{aligned}
& \Phi_{\mathscr{E}}: \mathbf{D}^{b}(A) \longrightarrow \mathbf{D}^{b}(B), \\
& \Phi_{\mathscr{F}}: \mathbf{D}^{b}(B) \longrightarrow \mathbf{D}^{b}(C)
\end{aligned}
$$

являются эквивалентностями. Обозначим через $\mathscr{G}$ объект в $\mathbf{D}^{b}(A \times C)$, которьй представляет композицию этих функторов.

Соотношение (10) дает нам изоморфизм $A d_{\mathscr{G}} \cong A d_{\mathscr{F}} \circ A d_{\mathscr{E}} \cdot$ И, следовательно, получаем, что

$\Phi_{\mathscr{J}(\mathscr{F})} \circ \Phi_{\mathscr{J}(\mathscr{E})} \cong\left(\Phi_{S_{A}}^{-1} \circ A d_{\mathscr{F}} \circ \Phi_{S_{A}}\right) \circ\left(\Phi_{S_{A}}^{-1} \circ A d_{\mathscr{E}} \circ \Phi_{S_{A}}\right) \cong \Phi_{S_{A}}^{-1} \circ A d_{\mathscr{G}} \circ \Phi_{S_{A}} \cong \Phi_{\mathscr{J}(\mathscr{G})}$. По теореме 5.1.7 все объекты $\mathscr{J}(\mathscr{E}), \mathscr{J}(\mathscr{F}), \mathscr{J}(\mathscr{G})$ являются линейными расслоениями, сосредоточенными на графиках изоморфизмов $f_{\mathscr{E}}, f_{\mathscr{F}}, f_{\mathscr{G}}$. Таким образом, получается равенство $f_{\mathscr{G}}=f_{\mathscr{F}} \cdot f_{\mathscr{E}}$.

СлеДСТВИЕ 5.1.13. Пусть $A-$ абелево многообразие и $\Phi_{\mathscr{E}}-$ автоэквивалентность производной категории $\mathbf{D}^{b}(A)$. Тогда соответствие $\Phi_{\mathscr{E}} \mapsto f_{\mathscr{E}}$ задает гомоморфизм групп

$$
\gamma_{A}: \operatorname{Auteq} \mathbf{D}^{b}(A) \longrightarrow \operatorname{Aut}(A \times \widehat{A}) .
$$

Таким образом, имеется функтор $F: \mathfrak{D} \longrightarrow \mathfrak{A}$. Наша дальнейшая цель - описать его. Для этого необходимо выяснить, какие элементы из $\operatorname{Iso}(A \times \widehat{A}, B \times \widehat{B})$ могут быть реализованы как $f_{\mathscr{E}}$ для некоторого $\mathscr{E}$, а также ответить на вопрос, когда для двух эквивалентностей $\mathscr{E}_{1}$ и $\mathscr{E}_{2}$ имеется равенство $f_{\mathscr{E}_{1}}=f_{\mathscr{E}_{2}}$.

Рассмотрим произвольный морфизм $f: A \times \widehat{A} \longrightarrow B \times \widehat{B}$. Удобно записать его в виде матрищы

$$
\left(\begin{array}{ll}
\alpha & \beta \\
\gamma & \delta
\end{array}\right)
$$

где $\alpha$ - это морфизм из $A$ в $B, \beta$-из $\widehat{A}$ в $B, \gamma-$ из $A$ в $\widehat{B}$ и $\delta$-из $\widehat{A}$ в $\widehat{B}$. Каждьй морфизм $f$ определяет два других $\widehat{f}$ и $\tilde{f}$ из $B \times \widehat{B}$ в $A \times \widehat{A}$, имеюшие следующие матричные формы:

$$
\widehat{f}=\left(\begin{array}{cc}
\widehat{\delta} & \widehat{\beta} \\
\widehat{\gamma} & \widehat{\alpha}
\end{array}\right) ; \quad \tilde{f}=\left(\begin{array}{cc}
\widehat{\delta} & -\widehat{\beta} \\
-\widehat{\gamma} & \widehat{\alpha}
\end{array}\right) .
$$

Определим множество $U(A \times \widehat{A}, B \times \widehat{B})$ как подмножество в $\operatorname{Iso}(A \times \widehat{A}, B \times \widehat{B})$, состоящее из таких $f$, что $\widetilde{f}$ совпадает с обратным к $f$, т.е.

$$
U(A \times \widehat{A}, B \times \widehat{B}):=\left\{f \in \operatorname{Iso}(A \times \widehat{A}, B \times \widehat{B}) \mid \widetilde{f}=f^{-1}\right\} .
$$

Если $B=A$, то это множество будем обозначать $U(A \times \widehat{A})$. Отметим, что $U(A \times \widehat{A})$ является подгруппой в $\operatorname{Aut}(A \times \widehat{A})$. 
ОПРЕДЕЛЕНИЕ 5.1.14. Назовем изоморфизм $f: A \times \widehat{A} \stackrel{\sim}{\longrightarrow} B \times \widehat{B}$ изометричнылм, если он принадлежит $U(A \times \widehat{A}, B \times \widehat{B})$.

ПРЕДЛОЖЕНИЕ 5.1.15. Для всякой әквивалентности $\Phi_{\mathscr{E}}: \mathbf{D}^{b}(A) \stackrel{\sim}{\longrightarrow} \mathbf{D}^{b}(B)$ изоморфизм $f_{\mathscr{E}}$ является изометричным.

ДокАЗАТЕльство. Переходя к алгебраическому замыканию, если это необходимо, мы можем предполагать, что поле $k$ алгебраически замкнуто. Для проверки равенства $\widetilde{f}_{\mathscr{E}}=f_{\mathscr{E}}^{-1}$ достаточно установить совпадение этих морфизмов на замкнутых точках. Пусть $f_{\mathscr{E}}$ переводит точку $(a, \alpha) \in A \times \widehat{A}$ в точку $(b, \beta) \in B \times \widehat{B}$. Мы должны показать, что $\widetilde{f}_{\mathscr{E}}(b, \beta)=(a, \alpha)$, или, что то же самое, показать, что $\widehat{f}(-b, \beta)=(-a, \alpha)$.

Изоморфизм $f_{\mathscr{E}}$ задается абелевым подмногообразием $X \hookrightarrow A \times \widehat{A} \times B \times \widehat{B}$. Следовательно, надо проверить, что $\mathscr{P}_{(0,0, \beta,-b)} \otimes \mathscr{O}_{X} \cong \mathscr{P}_{(\alpha,-a, 0,0)} \otimes \mathscr{O}_{X}$. Или, что эквивалентно, показать, что пучок

$$
\mathscr{J}^{\prime}:=\mathscr{P}_{(-\alpha, a, \beta,-b)} \otimes \mathscr{J}(\mathscr{E})
$$

изоморфен пучку $\mathscr{J}(\mathscr{E})$.

По предложению 5.1.11 функтор, задаваемьй пучком $\mathscr{J}^{\prime}$, является композицией функторов, представленных объектами $\mathscr{J}\left(S_{(-a,-\alpha)}\right), \mathscr{J}(\mathscr{E})$ и $\mathscr{J}\left(S_{(b, \beta)}\right)$. Таким образом, $\mathscr{J}^{\prime}$ совпадает с $\mathscr{J}\left(\mathscr{E}^{\prime}\right)$, где $\mathscr{E}^{\prime}$ - объект из $\mathbf{D}^{b}(A \times B)$, который представляет функтор

$$
\Phi_{(b, \beta)} \circ \Phi_{\mathscr{E}} \circ \Phi_{(-a,-\alpha)} \cdot
$$

А эта композиция по следствию 5.1 .10 изоморфна функтору $\Phi_{\mathscr{E}}$. То есть объект $\mathscr{E}$ изоморфен $\mathscr{E}$, и, значит, $\mathscr{J}^{\prime}=\mathscr{J}(\mathscr{E} \prime) \cong \mathscr{J}(\mathscr{E})$.

Как следствие теоремы 5.1.7 и предложения 5.1.15 получаем следуюший результат.

Теорема 5.1.16. Пусть $A$ и $B$-два абелевых многообразия над полем $k$. Если производные категории когерентных пучков $\mathbf{D}^{b}(A)$ и $\mathbf{D}^{b}(B)$ әквивалентны как триангулированные категории, тогда меэлду $A \times \widehat{A}$ и $B \times \widehat{B}$ существует изометричный изоморфизм.

Обратное утверждение также верно для абелевых многообразий над алгебраически замкнутым полем характеристики 0 и доказано в [38]. В разделе 5.3 дается другое доказательство этого факта.

СлЕДСТВИЕ 5.1.17. Для любого абелева многообразия А существует только конечное число неизоморфных абелевых многообразий, производные категории когерентных пучков которых әквивалентны категории $\mathbf{D}^{b}(A)$ (как триангулированные категории).

ДокАЗАТЕльство. В статье [26] доказано, что для каждого абелева многообразия $Z$ существует с точностью до изоморфизма только конечное число абелевых многообразий, допускающих вложение в $Z$ в качестве абелева подмногообразия. Применяя это утверждение к $Z=A \times \widehat{A}$ и используя теорему 5.1 .16 , получаем требуемый результат. 
5.2. Объекты, представляющие эквивалентности, и группы автоэквивалентностей. Из предложений 5.1.12 и 5.1.15 следует существование гомоморфизма из групшы точных автоэквивалентностей Auteq $\mathbf{D}^{b}(A)$ в группу изометричных автоморфизмов $U(A \times \widehat{A})$. В этом разделе дается описание ядра этого гомоморфизма. Нам уже известно из предложения 5.1.11, что все эквивалентности $\Phi_{(a, \alpha)}[n]$ принадлежат ядру. Покажем, что они в точности и составляют это ядро. Для доказательства этого факта нам понадобится утверждение, которое само по себе представляет интерес. Будет показано, что в случае абелевых многообразий если функтор $\Phi_{\mathscr{E}}$ является эквивалентностью, то объект $\mathscr{E}$ на самом деле есть пучок на произведении, с точностью до сдвига в производной категории. Отметим, что, например, для К3 поверхностей это не верно.

Лемма 5.2.1. Пусть $\mathscr{E}$ - оббект на произведении $A \times B$, который задает эквивалентность $\Phi_{\mathscr{E}}: \mathbf{D}^{b}(A) \longrightarrow \mathbf{D}^{b}(B)$. Рассмотрим проекцию $q:(A \times \widehat{A}) \times(B \times \widehat{B})$ $\longrightarrow A \times B$ и обозначим через $K$ прямой образ $\mathbf{R} q_{*} \mathscr{J}(\mathscr{E})$, где $\mathscr{J}(\mathscr{E})$ - облект, определенный в 5.1.6. Тогда $K$ изоморфен обгекту $\mathscr{E} \otimes\left(\left.\mathscr{E} \vee\right|_{(0,0)}\right)$, где $\left.\mathscr{E} \vee\right|_{(0,0)}$ обозначение для комплекса векторных пространств, который есть обратный образ обгекта $\mathbf{R}: \underline{\mathscr{H} о т}\left(\mathscr{E}, \mathscr{O}_{A \times B}\right)$ при вложении точки $(0,0)$ в абелево многообразие $A \times B$.

ДокаЗАТЕЛЬСтво. Рассмотрим абелево многообразие

$$
Z=(A \times \widehat{A}) \times(A \times A) \times(B \times B) \times(B \times \widehat{B})
$$

и объект

$$
H=p_{1234}^{*} S_{A} \otimes p_{35}^{*} \mathscr{E}^{\vee}[n] \otimes p_{46}^{*} \mathscr{E} \otimes p_{5678}^{*} S_{B}^{\vee}[2 n] .
$$

Из предложения 2.1.2 о композиции функторов и диаграммы $(51)$ следует, что $\mathscr{J}(\mathscr{E}) \cong$ $p_{1278 *} H$, и, значит, объект $K$ есть $p_{17 *} H$. Чтобы вычислить последний, сначала рассмотрим проекцию $Z$ на

$$
V=A \times(A \times A) \times(B \times B) \times B
$$

и обозначим ее через $v$. Теперь, чтобы вьгислить $v_{*} H$, вспомним, что функтор $\Phi_{S_{A}}-$ это композиция $\Phi_{\mathrm{P}_{A}}$ и $\mathbf{R} \mu_{A *}$, где

$$
\mathrm{P}_{A}=p_{14}^{*} \mathscr{O}_{\Delta} \otimes p_{23}^{*} \mathscr{P} \in \mathbf{D}^{b}((A \times \widehat{A}) \times(A \times A)) .
$$

Легко видеть, что $p_{134 *} \mathrm{P}_{A} \cong \mathscr{O}_{T_{A}}[-n]$, где $T \subset A \times A \times A$ - подмногообразие, изоморфное $A$ и состояшее из точек $(a, 0, a)$. Далее, учитьвая равенство $\mu_{A}\left(a_{1}, a_{2}\right)=$ $\left(a_{1}, m\left(a_{1}, a_{2}\right)\right)$, находим, что $p_{134 *} S_{A}$ также изоморфен $\mathscr{O}_{T_{A}}[-n]$. Аналогично проверяется равенство $p_{134 *} S_{B}^{\vee}[2 n]=\mathscr{O}_{T_{B}}$.

Таким образом, имеем, что

$$
v_{*} H \cong p_{123}^{*} \mathscr{O}_{T_{A}} \otimes p_{24}^{*} \mathscr{E}^{\vee} \otimes p_{35}^{*} \mathscr{E} \otimes p_{456}^{*} \mathscr{O}_{T_{B}}
$$

на $V$. Рассмотрим вложение

$$
j: A \times A \times B \times B \longrightarrow V, \quad\left(a_{1}, a_{2}, b_{1}, b_{2}\right) \mapsto\left(a_{1}, 0, a_{2}, 0, b_{1}, b_{2}\right) .
$$

Объект $v_{*} H$ изоморфен $j_{*} \mathscr{M}$, где

$$
\mathscr{M}=\left(\left.\mathscr{E}^{\vee}\right|_{(0,0)}\right) \otimes p_{12}^{*} \mathscr{O}_{\Delta_{A}} \otimes p_{23}^{*} \mathscr{E} \otimes p_{34}^{*} \mathscr{O}_{\Delta_{B}} .
$$

И окончательно получаем, что $K \cong p_{14 *} \mathscr{M} \cong\left(\left.\mathscr{E} \vee\right|_{(0,0)}\right) \otimes \mathscr{E}$. 
ПРЕДЛОЖЕНИЕ 5.2.2. Пусть $A$ и $B$ - абелевы многообразия и $\mathscr{E}$ - обгект категории $\mathbf{D}^{b}(A \times B)$ такой, что функтор $\Phi_{\mathscr{E}}: \mathbf{D}^{b}(A) \stackrel{\sim}{\longrightarrow} \mathbf{D}^{b}(B)$ есть точная әквивалентность. Тогда $\mathscr{E}$ имеет только одну нетривиальную когомологию, т.е. он изоморфен обгекту $\mathscr{F}[n]$, где $\mathscr{F}$ является пучком на $A \times B$.

ДокАЗАТЕЛЬСтво. Рассмотрим проекцию

$$
q:(A \times \widehat{A}) \times(B \times \widehat{B}) \longrightarrow A \times B
$$

и обозначим через $q^{\prime}$ ее ограничение на абелево подмногообразие $X$, которое есть носитель пучка $\mathscr{J}(\mathscr{E})$ и график изоморфизма $f_{\mathscr{E}}$. По теореме 5.1 .7 пучок $\mathscr{J}(\mathscr{E})$ есть $i_{*}(L)$, где $L$ - линейное расслоение на $X$.

Обозначим через $K$ объект $\mathbf{R} \cdot q_{*} \mathscr{J}(\mathscr{E})=\mathbf{R} \cdot q_{*}^{\prime} L$. Морфизм $q^{\prime}$ является гомоморфизмом абелевых многообразий. Пусть $d-$ это размерность $\operatorname{Ker}\left(q^{\prime}\right)$. Тогда $\operatorname{dim} \operatorname{Im}\left(q^{\prime}\right)=2 n-d$, и, значит, пучки когомологий $H^{j}(K)$ тривиальны для $j \notin[0, d]$.

С другой стороны, по лемме 5.2.1 объект $K$ изоморфен $\mathscr{E} \otimes\left(\left.\mathscr{E} \vee\right|_{(0,0)}\right)$.

Сдвигая, если необходимо, объект $\mathscr{E}$ в производной категории, мы можем предполагать, что самая правая ненулевая когомология $\mathscr{E}$ есть $H^{0}(\mathscr{E})$. Пусть $H^{-i}(\mathscr{E}), i \geqslant 0$, - это крайняя слева ненулевая когомология $\mathscr{E}$, а $H^{k}(\mathscr{E} \vee)$ - старшая ненулевая когомология объекта $\mathscr{E} \vee$. Заменяя, если необходимо, $\mathscr{E}$ на $T_{(a, b)}^{*} \mathscr{E}$, можно считать, что точка $(0,0)$ принадлежит носителю пучка $H^{k}\left(\mathscr{E}^{\vee}\right)$. Так как носитель $\mathscr{E}$ совпадает с носителем $K$, то носители всех пучков когомологий $\mathscr{E}$ принадлежат $\operatorname{Im}\left(q^{\prime}\right)$. В частности, имеем неравенство codim $\operatorname{Supp} H^{-i}(\mathscr{E}) \geqslant d$. Следовательно, все когомологии объекта $\left(H^{-i}(\mathscr{E})\right)^{\vee}[-i]$ степени, меньшей $i+d$, тривиальны.

Канонический морфизм $H^{-i}(\mathscr{E})[i] \longrightarrow \mathscr{E}$ индуцирует нетривиальньй морфизм

$$
\mathscr{E}^{\vee} \longrightarrow\left(H^{-i}(\mathscr{E})\right)^{\vee}[-i]
$$

Так как номера нетривиальных когомологий второго объекта принадлежат лучу $[i+d, \infty)$, получаем, что $k \geqslant i+d$, где $H^{k}(\mathscr{E} \vee)$, как и раньше, - старшая ненулевая когомология $\mathscr{E} \vee$. Таким образом, объект

$$
K=\left.\mathscr{E}^{\vee}\right|_{(0,0)} \otimes \mathscr{E}
$$

имеет нетривиальную когомологию с тем же самым номером $k \geqslant i+d$. С другой стороны, нам уже известно, что все пучки когомологий $H^{j}(K)$ тривиальны для $j \notin$ $[0, d]$. Это возможно только при условии $i=0$. И значит, объект $\mathscr{E}$ имеет только одну нетривиальную когомологию с номером 0 , следовательно, он изоморфен пучку.

Рассмотрим теперь случай $B \cong A$. Пусть $\mathscr{E}$-пучок на $A \times A$ такой, что $\Phi_{\mathscr{E}}$ является автоэквивалентностью. Мы хотим описать все такие $\mathscr{E}$, для которых $f_{\mathscr{E}}$ - тождественное отображение, т.е. его график $X$ является диагональю в $(A \times \widehat{A}) \times(A \times \widehat{A})$. Таким образом, получаем, что объект

$$
K=\left.\mathscr{E}^{\vee}\right|_{(0,0)} \otimes \mathscr{E}=\mathbf{R} \cdot q_{*} \mathscr{J}(\mathscr{E})
$$

имеет вид $\Delta_{*}(\mathscr{M})$, где $\mathscr{M}$ - объект на $A$, а $\Delta: A \longrightarrow A \times A$ - диагональное вложение. 
Вначале предположим, что точка $(0,0)$ принадлежит носителю пучка $\mathscr{E}$. Следовательно, $\left.\mathscr{E} \vee\right|_{(0,0)}$ является нетривиальным комплексом векторных пространств. Тогда условие $K=\Delta_{*}(\mathscr{M})$ влечет сушествование пучка $E$ на $A$ такого, что $\mathscr{E} \cong \Delta_{*}(E)$. Следовательно, $\Phi_{\mathscr{E}}(\cdot) \cong E \otimes(\cdot)$. Так как $\Phi_{\mathscr{E}}-$ автоэквивалентность, то $E$ является линейным расслоением. Нетрудно проверить, что условие $f_{\mathscr{E}}=\mathrm{id}$ может вьполняться, только если $E \in \operatorname{Pic}^{0}(A)$.

Если точка $(0,0)$ не принадлежит $\operatorname{Supp} \mathscr{E}$, можно заменить $\mathscr{E}$ на пучок $\mathscr{E} \prime:=$ $T_{\left(a_{1}, a_{2}\right) *} \mathscr{E}$ так, что его носитель уже содержит $(0,0)$. Из предложения 5.1.11 следует, что $f_{\mathscr{E}}{ }_{\mathscr{E}^{\prime}}=f_{\mathscr{E}}$. Как было показано выше, имеется изоморфизм $\mathscr{E} \prime \cong \Delta_{*}\left(E^{\prime}\right)$, где $E^{\prime} \in \operatorname{Pic}^{0}(A)$. Следовательно, $\mathscr{E} \cong T_{\left(a_{1}-a_{2}, 0\right) *} \Delta_{*}\left(E^{\prime}\right)$. Таким образом, получается следствие.

ПРЕДЛОЖЕНИЕ 5.2.4. Пусть $A$ - абелево многообразие. Тогда ядро гомоморфизма

$$
\gamma_{A}: \text { Auteq } \mathbf{D}^{b}(A) \longrightarrow U(A \times \widehat{A})
$$

состоит из автоэквивалентностей вида $\Phi_{(a, \alpha)}[i]=T_{a *}(\cdot) \otimes \mathscr{P}_{\alpha}[i]$ u, следовательно, изоморфно группе $\mathbb{Z} \oplus(A \times \widehat{A})_{k}$, где $(A \times \widehat{A})_{k}$ - группа $k$-точек абелева многообразия $A \times \widehat{A}$.

СлЕДСТВИЕ 5.2.4. Пусть $A$ и $B$ - два абелевых многообразия, а $\mathscr{E}_{1}$ и $\mathscr{E}_{2}-$ обгекты на произведении $A \times B$, которые задают әквивалентности между производными категориями когерентных пучков. Тогда, если $f_{\mathscr{E}_{1}}=f_{\mathscr{E}_{2}}$, то

$$
\mathscr{E}_{2} \cong T_{a * \mathscr{E}_{1}} \otimes \mathscr{P}_{\alpha}[i]
$$

для некоторой $k$-точки $(a, \alpha) \in A \times \widehat{A}$.

5.3. Полуоднородные векторные расслоения. В предыдущих разделах было показано, что эквивалентность $\Phi_{\mathscr{E}}$ из $\mathbf{D}^{b}(A)$ в $\mathbf{D}^{b}(B)$ индуцирует изометричный изоморфизм многообразий $A \times \widehat{A}$ и $B \times \widehat{B}$. В этом разделе предполагается, что поле $k$ алгебраически замкнуто и $\operatorname{ch} \operatorname{ar}(k)=0$. И в этих предположениях, используя технику статьи [30] и результаты из [7], будет показано, что каждый изометричный изоморфизм $f: A \times \widehat{A} \longrightarrow B \times \widehat{B}$ может быть реализован таким образом. Тот факт, что существование изометричного изоморфизма между многообразиями $A \times \widehat{A}$ и $B \times \widehat{B}$ влечет эквивалентность производных категорий $\mathbf{D}^{b}(A)$ и $\mathbf{D}^{b}(B)$, был доказан в статье [38]. Мы, таким образом, даем другое доказательство этого результата.

Вначале напомним, что любое линейное расслоение $\mathscr{L}$ на абелевом многообразии $D$ дает отображение $\varphi \mathscr{L}$ из $D$ в $\widehat{D}$, которое посылает точку $d$ в точку, соответствующую расслоению $T_{d}^{*} \mathscr{L} \otimes \mathscr{L}^{-1} \in \operatorname{Pic}^{0}(D)$. Это соответствие задает вложение $\mathrm{NS}(D)$ в $\operatorname{Hom}(D, \widehat{D})$. Более того, известно, что отображение $\varphi: D \longrightarrow \widehat{D}$ принадлежит образу $\mathrm{NS}(D)$ тогда и только тогда, когда $\widehat{\varphi}=\varphi$.

Полуоднородные расслоения на абелевом многообразии позволяют обобшить описанный вьше феномен следуюшим образом. С каждым элементом из $\mathrm{NS}(D) \otimes \mathbb{Q}$ можно связать некоторое соответствие на $D \times \widehat{D}$, и любое такое соответствие получается из полуоднородного расслоения (см. предложение 5.3.6 и лемму 5.1.10 ниже).

Вначале напомним определения однородных и полуоднородных расслоений на абелевых многообразиях и некоторые факты про них. 
ОПРЕДЕЛЕНИЕ 5.3.1. Векторное расслоение $\mathscr{E}$ на абелевом многообразии $D$ называется однородным, если $T_{d}^{*}(\mathscr{E}) \cong \mathscr{E}$ для каждой точки $d \in D$.

ОПреДЕлЕниЕ 5.3.2. Векторное расслоение $\mathscr{F}$ на абелевом многообразии $D$ называется унипотентным, если существует фильтрация

$$
0=\mathscr{F}_{0} \subset \mathscr{F}_{1} \subset \cdots \subset \mathscr{F}_{n}=\mathscr{F}
$$

такая, что $\mathscr{F}_{i} / \mathscr{F}_{i-1} \cong \mathscr{O}_{D}$ для всех $i=1, \ldots, n$.

Следующее предложение дает характеризацию однородных векторных расслоений.

ПРЕДЛОЖЕНИЕ 5.3 .3 [28], [30]. Пусть $\mathscr{E}$ - векторное расслоение на абелевом многообразии D. Тогда следующие условия әквивалентны:

(i) $\mathscr{E}-$ однородное,

(ii) существуют линейные расслоения $\mathscr{P}_{i} \in \mathrm{Pic}^{0}(D)$ и унипотентные расслоения $\mathscr{F}_{i}$ такие, что $\mathscr{E} \cong \bigoplus_{i}\left(\mathscr{F}_{i} \otimes \mathscr{P}_{i}\right)$.

ОПРЕДЕЛЕНИЕ 5.3.4. Векторное расслоение $\mathscr{E}$ на абелевом многообразии $D$ называется полуоднородным, если для каждой точки $d \in D$ существует линейное расслоение $\mathscr{L}$ на $D$ такое, что $T_{d}^{*}(\mathscr{E}) \cong \mathscr{E} \otimes \mathscr{L}$. (Отметим, что $\mathscr{L}$ в этом случае принадлежит $\operatorname{Pic}^{0}(D)$.)

Напомним, что векторное расслоение на многообразии назьвается простым, если его алгебра эндоморфизмов совпадает с полем $k$.

Следуюшее утверждение доказано в [30].

ПРЕДЛОЖЕНИЕ 5.3.5 [30; теорема 5.8]. Пусть $\mathscr{E}$ - простое векторное расслоение на абелевом многообразии D. Тогда следующие условия әквивалентнь:

(1) $\operatorname{dim} \mathrm{H}^{j}(D, \underline{\mathscr{E} n d}(\mathscr{E}))=\left(\begin{array}{c}n \\ j\end{array}\right)$ для любого $j=0, \ldots, n$,

(2) $\mathscr{E}$ - полуоднородное расслоение,

(3) $\mathscr{E}$ 站 $(\mathscr{E})$ - однородное расслоение,

(4) существуют изогения $\pi: Y \longrightarrow D$ и линейное расслоение $\mathscr{L}$ на $Y$ такие, что $\mathscr{E} \cong \pi_{*}(\mathscr{L})$.

Пусть $\mathscr{E}$ - векторное расслоение на абелевом многообразии $D$. Обозначим через $\mu(\mathscr{E})$ класс эквивалентности $\frac{\operatorname{det}(\mathscr{E})}{r(\mathscr{E})}$ в $\mathrm{NS}(D) \otimes_{\mathbb{Z}} \mathbb{Q}$.

С любым элементом $\mu=\frac{[\mathscr{L}]}{l} \in \mathrm{NS}(D) \otimes_{\mathbb{Z}} \mathbb{Q}$, и значит, с любым расслоением $\mathscr{E}$, можно связать некоторое соответствие $\Phi_{\mu} \subset D \times \widehat{D}$, заданное по правилу $\Phi_{\mu}=$ $\operatorname{Im}\left[D \stackrel{\left(l, \varphi_{\mathscr{L}}\right)}{\longrightarrow} D \times \widehat{D}\right]$, где $\varphi_{\mathscr{L}}-$ хорошо известное отображение из $D$ в $\widehat{D}$, которое посылает точку $d$ в точку, соответствующую расслоению $T_{d}^{*} \mathscr{L} \otimes \mathscr{L}^{-1} \in \operatorname{Pic}^{0}(D)$. Обозначим через $q_{1}$ и $q_{2}$ проекции $\Phi_{\mu}$ на $D$ и $\widehat{D}$ соответственно. В частном случае, когда расслоение является линейным расслоением $\mathscr{L}$, получается график отображения $\varphi \mathscr{L}: D \longrightarrow \widehat{D}$.

В работе [30] дается полное описание всех простых полуоднородных расслоений. 


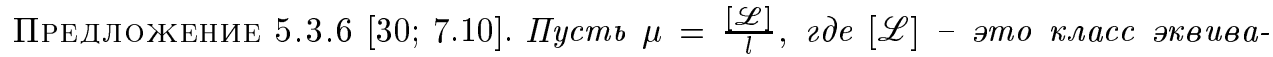
лентности расслоения $\mathscr{L}$ в $\mathrm{NS}(D)$ и l некоторое полохсительное целое число. Тогда

(1) существует простое полуоднородное векторное расслоение Е с наклоном $\mu(\mathscr{E})=\mu$;

(2) всякое простое полуоднородное векторное расслоение हू' с наклоном $\mu\left(\mathscr{E}^{\prime}\right)=\mu$ имеет вид $\mathscr{E} \otimes \mathscr{M}$ для некоторого линейного расслоения $\mathscr{M} \in \operatorname{Pic}^{0}(D)$

(3) выполнены равенства $r(\mathscr{E})^{2}=\operatorname{deg}\left(q_{1}\right)$ и $\chi(\mathscr{E})^{2}=\operatorname{deg}\left(q_{2}\right)$.

Следующее утверждение позволяет охарактеризовать все полуоднородные векторные расслоения в терминах простых расслоений.

ПРЕДЛОЖЕНИЕ 5.3 .7 [30; 6.15,6.16]. Всякое полуоднородное векторное расслоение $\mathscr{F}$ с наклоном $\mu$ имеет фильтрацию

$$
0=\mathscr{F}_{0} \subset \mathscr{F}_{1} \subset \cdots \subset \mathscr{F}_{n}=\mathscr{F}
$$

такую, что $\mathscr{E}_{i}=\mathscr{F}_{i} / \mathscr{F}_{i-1}-$ простые полуоднородные векторные расслоения с тем же самым наклоном $\mu$. И всякое простое полуоднородное расслоение стабильно.

Следующие две леммы про полуоднородные расслоения, которые являются прямыми следствиями предыдуших утверждений, будут полезны нам в дальнейшем.

Лемма 5.3.8. Два простых полуоднородных расслоения $\mathscr{E}_{1}$ u $\mathscr{E}_{2}$ с одним $и$ тем же наклоном $\mu$ либо изоморфны, либо ортогональнь друг другу, т.е. либо $\mathscr{E}_{1}=\mathscr{E}_{2}$, либo

$$
\operatorname{Ext}^{i}\left(\mathscr{E}_{1}, \mathscr{E}_{2}\right)=0, \quad \operatorname{Ext}^{i}\left(\mathscr{E}_{2}, \mathscr{E}_{1}\right)=0
$$

для всех $i$.

ДоКАЗАТЕЛЬСТВо. Из предложения 5.3.6 следует, что $\mathscr{E}_{2} \cong \mathscr{E}_{1} \otimes \mathscr{M}$, и, следовательно, $\underline{\mathscr{H} о \mathrm{~m}}\left(\mathscr{E}_{1}, \mathscr{E}_{2}\right)$ является однородным расслоением. Всякое однородное расслоение по предложению 5.3.3 представляется в виде суммы унипотентных расслоений, подкрученных на линейные из $\operatorname{Pic}^{0}(D)$. Поэтому либо все когомологии $\underline{\mathscr{H} o m}\left(\mathscr{E}_{1}, \mathscr{E}_{2}\right)$ равны нулю и, значит, расслоения $\mathscr{E}_{1}$ и $\mathscr{E}_{2}$ ортогональны, либо у $\underline{\mathscr{H} o m}\left(\mathscr{E}_{1}, \mathscr{E}_{2}\right)$ существует ненулевое сечение. В последнем случае получаем ненулевой гомоморфизм из $\mathscr{E}_{1}$ в $\mathscr{E}_{2}$. Но эти два расслоения стабильны и имеют одинаковый наклон. Значит, любой ненулевой гомоморфизм на самом деле является изоморфизмом.

ЛЕмМА 5.3.9. Пусть $\mathscr{E}$ - простое полуоднородное векторное расслоение на абелевом многообразии D. Тогда $T_{d}^{*}(\mathscr{E}) \cong \mathscr{E} \otimes \mathscr{P}_{\delta}$, если и только если $(d, \delta) \in \Phi_{\mu}$.

ДокАЗАТЕльство. Сначала покажем, что для каждой точки $(d, \delta) \in \Phi_{\mu}$ имеется изоморфизм $T_{d}^{*}(\mathscr{E}) \cong \mathscr{E} \otimes \mathscr{P}_{\delta}$. Действительно, положим $l=r(\mathscr{E})$ и $\mathscr{L}=\operatorname{det}(\mathscr{E})$. Мы знаем, что по определению $\Phi_{\mu}$ можно записать $(d, \delta)=(l x, \varphi \mathscr{L}(x))$ для некоторой точки $x \in D$. Так как $\mathscr{E}$ полуоднородно, найдется линейное расслоение $\mathscr{M} \in \mathrm{Pic}^{0}(D)$ такое, что

$$
T_{x}^{*}(\mathscr{E}) \cong \mathscr{E} \otimes \mathscr{M}
$$


Сравнивая детерминанты, получаем равенство $T_{x}^{*}(\mathscr{L}) \cong \mathscr{L} \otimes \mathscr{M}^{\otimes l}$. По определению отображения $\varphi_{\mathscr{L}}$, это значит, что $\mathscr{P}_{\varphi_{\mathscr{L}}(x)}=\mathscr{M}^{\otimes} l$. С другой стороны, итерируя равенство (53) $l$ раз, получаем

$$
T_{l x}^{*}(\mathscr{E}) \cong \mathscr{E} \otimes \mathscr{M}^{\otimes l}=\mathscr{E} \otimes \mathscr{P}_{\varphi \mathscr{L}(x)}
$$

И, следовательно, $T_{d}^{*}(\mathscr{E}) \cong \mathscr{E} \otimes \mathscr{P}_{\delta}$, так как $(d, \delta)=(l x, \varphi \mathscr{L}(x))$.

В обратную сторону. Давайте введем подгруппу $\Sigma^{0}(\mathscr{E}) \subset \widehat{D}$, заданную условием

$$
\Sigma^{0}(\mathscr{E}):=\left\{\delta \in \widehat{D} \mid \mathscr{E} \otimes \mathscr{P}_{\delta} \cong \mathscr{E}\right\}
$$

Так как $\mathscr{E}$ полуоднородно, то расслоение $\underline{\mathscr{E} n d}(\mathscr{E})$ однородно по предложению 5.3.5. Таким образом, $\underline{\mathscr{E} n d}(\mathscr{E})$ можно представить как сумму $\bigoplus_{i}\left(\mathscr{F}_{i} \otimes \mathscr{P}_{i}\right)$, где все $\mathscr{F}_{i}$ унипотентны. Следовательно, $\mathrm{H}^{0}(\underline{\mathscr{E} n d}(\mathscr{E}) \otimes \mathscr{P}) \neq 0$ не более чем для $r^{2}$ линейных расслоений $\mathscr{P} \in \mathrm{Pic}^{0}(D)$. То есть порядок групшы $\Sigma^{0}(\mathscr{E})$ не больше $r^{2}$. С другой стороны, известно, что $q_{2}\left(\operatorname{Ker}\left(q_{1}\right)\right) \subset \Sigma^{0}(\mathscr{E})$. Следовательно, получаем равенства ord $\Sigma^{0}(\mathscr{E})=r^{2}$ и $q_{2}\left(\operatorname{Ker}\left(q_{1}\right)\right)=\Sigma^{0}(\mathscr{E})$.

Предположим теперь, что $T_{d}^{*}(\mathscr{E}) \cong \mathscr{E} \otimes \mathscr{P}_{\delta}$ для некоторой точки $(d, \delta) \in D \times \widehat{D}$. Рассмотрим некоторую точку $\delta^{\prime} \in \widehat{D}$ такую, что $\left(d, \delta^{\prime}\right) \in \Phi_{\mu}$. Как уже было показано, в этом случае имеется изоморфизм $T_{d}^{*}(\mathscr{E}) \cong \mathscr{E} \otimes \mathscr{P}_{\delta^{\prime}}$. Следовательно, $\mathscr{E} \otimes \mathscr{P}_{\left(\delta-\delta^{\prime}\right)} \cong \mathscr{E}$, и, значит, $\left(\delta-\delta^{\prime}\right) \in \Sigma^{0}(\mathscr{E})$. Но так как $\Sigma^{0}(\mathscr{E})=q_{2}\left(\operatorname{Ker}\left(q_{1}\right)\right)$, то точка $\left(0, \delta-\delta^{\prime}\right)$ принадлежит $\Phi_{\mu}$. И значит, точка $(d, \delta)$ также принадлежит $\Phi_{\mu}$.

Теперь приведем конструкцию, которая показьвает, как по изометричному изоморфизму $f$ можно построить объект $\mathscr{E}$ на произведении такой, что он задает эквивалентность производных категорий, и для которого $f_{\mathscr{E}}$ совпадает с $f$.

КонстрУкция 5.3.10. Зафиксируем изометричньй изоморфизм $f: A \times \widehat{A} \longrightarrow$ $B \times \widehat{B}$. Обозначим через $\Gamma$ его график. Изоморфизм $f$, как и раньше, будем записьвать в матричной форме

$$
f=\left(\begin{array}{cc}
x & y \\
z & w
\end{array}\right)
$$

Мы предположим, что $y: \widehat{A} \longrightarrow B$ является изогенией. В этом случае можно сопоставить отображению $f$ элемент $g \in \operatorname{Hom}(A \times B, \widehat{A} \times \widehat{B}) \otimes_{\mathbb{Z}} \mathbb{Q}$, которьй имеет следуюшую форму:

$$
g=\left(\begin{array}{cc}
y^{-1} x & -y^{-1} \\
-\widehat{y}^{-1} & w y^{-1}
\end{array}\right)
$$

Элемент $g$ задает некоторое соответствие на $(A \times B) \times(\widehat{A} \times \widehat{B})$. Легко проверить, что изометричность $f$ влечет равенство $\widehat{g}=g$. Это значит, что элемент $g$ на самом деле принадлежит образу $\mathrm{NS}(A \times B) \otimes_{\mathbb{Z}} \mathbb{Q}$ при каноническом вложении в $\operatorname{Hom}(A \times B$, $\widehat{A} \times \widehat{B}) \otimes_{\mathbb{Z}} \mathbb{Q}\left(\right.$ см., например, [32]). Следовательно, сушествует $\mu=\frac{[\mathscr{L}]}{l} \in \mathrm{NS}(A \times B)$ такое, что $\Phi_{\mu}$ совпадает с графиком соответствия $g$. Предложение 5.3.6 говорит нам, что по каждому $\mu$ можно построить простое полуоднородное расслоение $\mathscr{E}$ на $A \times B$ с наклоном $\mu(\mathscr{E})=\mu$. 
Чуть ниже будет показано, что функтор $\Phi_{\mathscr{E}}$ из $\mathbf{D}^{b}(A)$ в $\mathbf{D}^{b}(B)$ является эквивалентностью и $f_{\mathscr{E}}=f$. Но сначала давайте сравним графики $Г$ и $\Phi_{\mu}$. Если точка $(a, \alpha, b, \beta)$ принадлежит $\Gamma$, то

$$
\begin{aligned}
b & =x(a)+y(\alpha), \\
\beta & =z(a)+w(\alpha),
\end{aligned} \quad \text { и, следовательно, } \quad \begin{array}{ll}
\alpha=-y^{-1} x(a)+y^{-1}(b), \\
\beta & =\left(z-w y^{-1} x\right)(a)+w y^{-1}(b) .
\end{array}
$$

Изометричность $f$ влечет равенство $\left(z-w y^{-1} x\right)=-\widehat{y}^{-1}$. И значит, точка $(a, \alpha, b, \beta)$ принадлежит графику $Г$ тогда и только тогда, когда $(a,-\alpha, b, \beta)$ принадлежит $\Phi_{\mu}$. Таким образом, мы находим, что

$$
\Phi_{\mu}=\left(1_{A},-1_{\widehat{A}}, 1_{B}, 1_{\widehat{B}}\right) \Gamma
$$

В частности, из-за того, что $f$ изоморфизм, следует, что проекции $\Phi_{\mu}$ на $A \times \widehat{A}$ и $B \times \widehat{B}$ являются изоморфизмами.

ПРЕДЛОЖЕНИЕ 5.3.11. Пусть $\mathscr{E}$ - полуоднородное расслоение на $A \times B$, построенное по изометричному изоморфизму $f$ описанным выше способом. Тогда функтор $\Phi_{\mathscr{E}}: \mathbf{D}^{b}(A) \longrightarrow \mathbf{D}^{b}(B)$ является әквивалентностью.

ДокАЗАТЕЛЬСТВо. Обозначим через $\mathscr{E}_{a}$ ограничение расслоения $\mathscr{E}$ на слой $\{a\} \times B$. По теореме 2.1.5 для того, чтобы доказать, что $\Phi_{\mathscr{E}}$ вполне строгий, достаточно проверить, что все расслоения $\mathscr{E}_{a}$ простые и ортогональны друг другу для разных точек.

Во-первых, заметим, что ранг расслоения $\mathscr{E}$ равен по предложению 5.3.6 квадратному корню из степени отображения $\Phi_{\mu} \longrightarrow A \times B$, т.е. есть $\left.\sqrt{\operatorname{deg}(\beta}\right)$.

Из полуоднородности $\mathscr{E}$ немедленно следует, что все расслоения $\mathscr{E}_{a}$ также полуоднородны. Кроме того, наклон ограничения $\mu\left(\mathscr{E}_{a}\right)$ равен $\delta \beta^{-1} \in \mathrm{NS}(B) \otimes \mathbb{Q} \subset$ $\operatorname{Hom}(B, \widehat{B}) \otimes \mathbb{Q}$. Обозначим $\delta \beta^{-1}$ для краткости через $\nu$, рассматривая его как элемент $\mathrm{NS}(B) \otimes \mathbb{Q}$. Предложение 5.3.6 утверждает существование простого полуоднородного расслоения $\mathscr{F}$ на $B$ с данным наклоном $\mu(\mathscr{F})=\nu$. Очевидно, что $\Phi_{\nu}$ в этом случае есть $\operatorname{Im}[\widehat{A} \stackrel{(\beta, \delta)}{\longrightarrow} B \times \widehat{B}]$. Так как $f$-изоморфизм, то отображение $\widehat{A} \stackrel{(\beta, \delta)}{\longrightarrow} B \times \widehat{B}$ является вложением. Следовательно, опять применяя предложение 5.3.6, получаем равенство $r(\mathscr{F})=\sqrt{\operatorname{deg}(\beta)}=r\left(\mathscr{E}_{a}\right)$. Таким образом, два расслоения $\mathscr{F}$ и $\mathscr{E}_{a}$ полуоднородны и имеют одинаковые наклон и ранг. Кроме того, расслоение $\mathscr{F}$ простое. Из предложений 5.3.7 и 5.3.6 (2) следует, что $\mathscr{E}_{a}$ также простое расслоение.

Далее, из леммы 5.3 .8 следует, что расслоения $\mathscr{E}_{a_{1}}$ и $\mathscr{E}_{a_{2}}$ для двух точек $a_{1}, a_{2} \in A$ либо ортогональны, либо изоморфны. Предположим, что они изоморфны. Так как расслоение $\mathscr{E}$ полуоднородно, то

$$
T_{\left(a_{2}-a_{1}, 0\right)}^{*} \mathscr{E} \cong \mathscr{E} \otimes \mathscr{P}_{(\alpha, \beta)}
$$

для некоторой точки $(\alpha, \beta) \in \widehat{A} \times \widehat{B}$. В частности, получаем

$$
\mathscr{E}_{a_{2}} \otimes \mathscr{P}_{\beta} \cong \mathscr{E}_{a_{1}} \cong \mathscr{E}_{a_{2}}
$$

Следовательно, $\mathscr{P}_{\beta} \in \Sigma^{0}\left(\mathscr{E}_{a}\right)($ см. $(54))$. 
По лемме 5.3.9 и предложению 5.3.6 порядки $\Sigma^{0}(\mathscr{E})$ и $\Sigma^{0}\left(\mathscr{E}_{a}\right)$ равны $r^{2}$. Мы утверждаем, что естественное отображение $\sigma: \Sigma^{0}(\mathscr{E}) \longrightarrow \Sigma^{0}\left(\mathscr{E}_{a}\right)$ является изоморфизмом. Действительно, в противном случае нашлась бы точка $\alpha^{\prime} \in \widehat{A}$ такая, что $\mathscr{E} \otimes \mathscr{P}_{\alpha^{\prime}} \cong \mathscr{E}$. И по лемме 5.3 .9 тогда $\left(0, \alpha^{\prime}, 0,0\right) \in \Phi_{\mu}$. А это противоречит тому, что проекция $\Phi_{\mu} \longrightarrow B \times \widehat{B}$ есть изоморфизм.

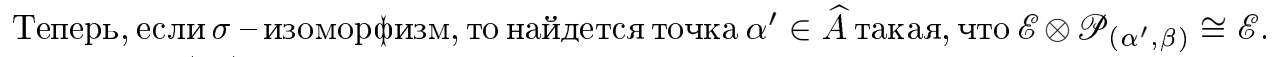
Из равенства (55) следует, что

$$
T_{\left(a_{2}-a_{1}, 0\right)}^{*} \mathscr{E} \cong \mathscr{E} \otimes \mathscr{P}\left(\alpha-\alpha^{\prime}, 0\right)
$$

По лемме 5.3.9 это значит, что точка $\left(a_{2}-a_{1}, \alpha-\alpha^{\prime}, 0,0\right)$ принадлежит $\Phi_{\mu}$. Опять, так как проекция $\Phi_{\mu} \longrightarrow B \times \widehat{B}$ есть изоморфизм, то получаем равенство $a_{2}-a_{1}=0$. Таким образом, для двух разных точек $a_{1}$ и $a_{2}$ расслоения $\mathscr{E}_{a_{1}}$ и $\mathscr{E}_{a_{2}}$ ортогональны. И, следовательно, функтор $\Phi_{\mathscr{E}}: \mathbf{D}^{b}(A) \longrightarrow \mathbf{D}^{b}(B)$ является вполне строгим. По тем же соображениям сопряженный функтор $\Psi_{\mathscr{E}} \vee$ также является вполне строгим. Следовательно, $\Phi_{\mathscr{E}}-$ эквивалентность.

ПРЕДЛОЖЕНИЕ 5.3.12. Пусть $\mathscr{E}$ - полуоднородное расслоение, построенное описанным выше способом по изометричному изоморфизму $f: A \times \widehat{A} \longrightarrow B \times \widehat{B}$. Тогда имеется равенство $f_{\mathscr{E}}=f$.

ДокаЗАТЕльство. Обозначим через $X$ график морфизма $f_{\mathscr{E}}$. Из следствия 5.1.10 следует, что точка $(a, \alpha, b, \beta)$ принадлежит $X$ тогда и только тогда, когда

$$
T_{b *} \mathscr{E} \otimes \mathscr{P}_{\beta} \cong T_{a}^{*} \mathscr{E} \otimes \mathscr{P}_{\alpha},
$$

что равносильно равенству

$$
T_{(a, b)}^{*} \mathscr{E} \cong \mathscr{E} \otimes \mathscr{P}_{(-\alpha, \beta)}
$$

Следовательно, по лемме 5.3.9 получаем, что $X=\left(1_{A},-1_{\widehat{A}}, 1_{B}, 1_{\widehat{B}}\right) \Phi_{\mu}$ где $\mu=\mu(\mathscr{E})$ это наклон $\mathscr{E}$. С другой стороны, по конструкции 5.3.10 график Г отображения $f$ также есть $\left(1_{A},-1_{\widehat{A}}, 1_{B}, 1_{\widehat{B}}\right) \Phi_{\mu}$. Значит, изоморфизмы $f_{\mathscr{E}}$ и $f$ совпадают.

При построении расслоения $\mathscr{E}$ по изоморфизму $f$ мы предположили, что отображение $y: \widehat{A} \longrightarrow B$ является изогенией. Если это не так, то представим $f$ как композицию двух отображений $f_{1} \in U(A \times \widehat{A}, B \times \widehat{B})$ и $f_{2} \in U(A \times \widehat{A})$, для которых $y_{1}$ и $y_{2}$ являются изогениями. Легко видеть, что это всегда можно сделать. Теперь для каждого $f_{i}$ найдем свой объект $\mathscr{E}_{i}$, далее рассмотрим композицию функторов $\Phi_{\mathscr{E}_{i}}$ и возьмем объект, ее представляющий. Утверждения, доказанные в этом и предыдущих разделах, мы можем объединить в следуюшие теоремы.

ТеОРема 5.3.13. Пусть $A$ и $B$-два абелевых многообразия над алгебраически замкнутым полем характеристики 0 . Тогда ограниченные производные категории когерентных пучков $\mathbf{D}^{b}(A)$ и $\mathbf{D}^{b}(B)$ эквивалентны как триангулированные категории тогда и только тогда, когда существует изометричный изоморфизм $f: A \times \widehat{A} \stackrel{\sim}{\longrightarrow} B \times \widehat{B}$. 
Теорема 5.3.14. Пусть $A$ - абелево многообразие над алгебраически замкнутылм полем характеристики 0. Тогда группа точных автоэквивалентностей производной категории Auteq $\mathbf{D}^{b}(A)$ может быть включена в следующую короткую точную последовательность групп:

$$
0 \longrightarrow \mathbb{Z} \oplus(A \times \widehat{A})_{k} \longrightarrow \text { Auteq } \mathbf{D}^{b}(A) \longrightarrow U(A \times \widehat{A}) \longrightarrow 1 .
$$

Таким образом, группа Auteq $\mathbf{D}^{b}(A)$ имеет нормальную подгруппу $(A \times \widehat{A})_{k}$, которая состоит из функторов вида $T_{a *}(\cdot) \otimes \mathscr{P}_{\alpha}$, где $(a, \alpha) \in A \times \widehat{A}$. Фактор по этой подгруппе является центральным расширением $U(A \times \widehat{A})$ с помощью $\mathbb{Z}$.

Это центральное расширение описьвается некоторьм 2-коциклом, формулу для которого можно найти в [37].

ПримеР 5.3.15. Рассмотрим абелево многообразие $A$, для которого кольцо эндоморфизмов $\operatorname{End}(A)$ изоморфно кольцу $\mathbb{Z}$. Тогда группа Нерона-Севери $\operatorname{NS}(A)$ изоморфна групше $\mathbb{Z}$. Обозначим через $\mathscr{L}$ и $\mathscr{M}$ образуюшие $\operatorname{NS}(A)$ и $\mathrm{NS}(\widehat{A})$ соответственно. Композиция $\varphi \mathscr{M} \circ \varphi_{\mathscr{L}}$ есть $N \cdot \mathrm{id}_{A}$ с некоторым $N>0$. В этом случае группа $U(A \times \widehat{A})$ совпадает с конгруенц-подгруппой $\Gamma_{0}(N) \subset \operatorname{SL}(2, \mathbb{Z})$. Далее, пусть $B-$ это другое абелево многообразие такое, что $B \times \widehat{B}$ изоморфин $A \times \widehat{A}$. Легко проверить, что любой такой изоморфизм является изометричным. Абелево многообразие $B$ можно представить как образ некоторого морфизма $A \stackrel{(k \cdot \mathrm{id}, m \varphi \mathscr{L})}{\longrightarrow} A \times \widehat{A}$. Можно считать, что НОД $(k, m)=1$. Обозначим через $\psi$ этот морфизм из $A$ в $B$. Ядро $\psi$ есть $\operatorname{Ker}\left(m \varphi_{\mathscr{L}}\right) \cap A_{k}$. Так как НОД $(k, m)=1$, то на самом деле $\operatorname{Ker}(\psi)=\operatorname{Ker}(\varphi \mathscr{L}) \cap A_{k}$. $\mathrm{C}$ другой стороны, имеет место включение $\operatorname{Ker}(\varphi) \subset A_{N}$. Таким образом, без потери обшности можно считать, что $k$ является делителем $N$. Каждый $k$ - делитель $N-$ индуцирует абелево многообразие $B:=A /\left(\operatorname{Ker}(\varphi \mathscr{L}) \cap A_{k}\right)$. Очевидно, что два разных делителя $N$ - неизоморфные абелевы многообразия. Более того, легко проверить, что вложение $B$ в $A \times \widehat{A}$ расшепляется тогда и только тогда, когда $\mathrm{HOД}(k, N / k)=1$. Следовательно, число абелевых многообразий $B$ таких, что $\mathbf{D}^{b}(B) \simeq \mathbf{D}^{b}(A)$, совпадает с $2^{s}$, где $s$ - число простых делителей $N$.

\section{СПИСОК ЛИТЕРАТУРЫ}

[1] J. Backelin. On the rates of growth of the homologies of Veronese subrings // Lecture Notes in Math. 1985. V. 1183. P. 79-100.

[2] А. А. Бейлинсон. Когерентные пучки на $\mathbb{P}^{n}$ и проблемы линейной алгебры $/ /$ Функц. анализ и его прил. 1978. Т. 12. № 3. С. 68-69.

[3] A. Beı̌linson, J. Bernstein, P. Deligne. Faisceaux pervers // Astérisque. 1982. V. 100. P. 5-171.

[4] И. Бернштейн, И. М. Гельфанд, С. И. Гельфанд. Алгебраические векторные расслоения на $\mathbb{P}^{n}$ и проблемы линейной алгебры // Функц. анализ и его прил. 1978. Т. 12. № 3. C. $66-67$.

[5] А.И. Бондал. Представления ассоциативных алгебр и когерентные пучки // Изв. АН СССР. Сер. матем. 1989. Т. 53. №1. С. 25-44.

[6] А. И. Бондал, М. Капранов. Представимые функторы, функторы Серра и перестройки // Изв. АН СССР. Сер. матем. 1989. Т. 53. №6. С. 1183-1205.

[7] A. Bondal, D. Orlov. Semiorthogonal decomposition for algebraic varieties // Preprint. Bonn: Max-Planck-Institut für Mathematik, 1995; alg-geom/9506006.

[8] A. Bondal, D. Orlov. Reconstruction of a variety from the derived category and groups of autoequivalences // Compositio Math. 2001. V. 125. №3. P. 327-344. 
[9] T. Bridgeland. Equivalences of triangulated categories and Fourier-Mukai transforms // Bull. London Math. Soc. 1999. V. 31. № 1. P. 25-34.

[10] T. Bridgeland. Flops and derived categories // Invent. Math. 2002. V. 147. № 3. P. 613-632.

[11] А. Картан, С. Эйленберг. Гомологическая алгебра. М.: ИЛ, 1960.

[12] P. Deligne. Cohomologie à supports propres // Théorie des Topos et Cohomologie Étale des Schémas (SGA 4). Berlin: Springer-Verlag, 1973. P. 252-480. (Lecture Notes in Math. V. 305.)

[13] П. Габриель, М. Цисман. Категории частных и теория гомотопий. М.: Мир, 1971.

[14] С. И. Гельфанд, Ю. И. Манин. Методы гомологической алгебры. Введение в теорию когомологий и производных категорий. М.: Наука, 1988.

[15] A. Grothendieck. Sur quelques points d'algèbre homologique // Tohoku Math. J. (2). 1957. V. 9. P. 119-221.

[16] A. Grothendieck. Éléments de géométrie algébrique // Publ. Math. Inst. Hautes Études Sci. 1961-1967. № 4, 8, 11, 17, 20, 24, 28, 32 .

[17] R. Hartshorne. Residues and Duality. Berlin: Springer-Verlag, 1966. (Lecture Notes in Math. V. 20.)

[18] S. P. Inamdar, V. B. Mehta. Frobenius splitting of Schubert varieties and linear syzygies // Amer. J. Math. 1994. V. 116. №6. P. 1569-1586.

[19] В.А. Исковских. Лекции по трехмерным алгебраическим многообразиям: Многообразия Фано. М.: Изд-во Моск. ун-та, 1988.

[20] М. Капранов. Производная категория когерентных пучков на многообразии Грассмана // Изв. АН СССР. Сер. матем. 1984. Т. 48. № 1. С. 192-202.

[21] М. Капранов. Производная категория когерентных пучков на квадрике // Функц. анализ и его прил. 1986. Т. 20. № 2. С. 67.

[22] M. Kapranov. On the derived categories of coherent sheaves on some homogeneous spaces // Invent. Math. 1988. V. 92. № 2. P. 479-508.

[23] M. Kashiwara. The Riemann-Hilbert problem for holonomic systems // Publ. Res. Inst. Math. Sci. 1984. V. 20. № 2. P. 319-365.

[24] М. Кашивара, П. Шапира. Пучки на многообразиях. М.: Мир, 1994.

[25] B. Keller. Derived categories and their uses // Handbook of Algebra / ed. M. Hazewinkel. V. 1. Amsterdam: North-Holland, 1996. P. 671-701.

[26] H. W. Lenstra, Jr, F. Oort, Yu. G. Zarhin. Abelian subvarieties // J. Algebra. 1996. V. 180. P. 513-516.

[27] E. Looijenga, C. Peters. Torelli theorem for Kähler K3 surfaces // Compositio Math. 1980/81. V. 42. № 2. P. 145-186.

[28] M. Miyanishi. Some remarks on algebraic homogeneous vector bundles // Number Theory, Algebraic Geometry and Commutative Algebra. Tokyo: Kinokuniya, 1973. P. 71-93.

[29] S. Mukai. Duality between $D(X)$ and $D(\widehat{X})$ with its application to Picard sheaves // Nagoya Math. J. 1981. V. 81. P. 153-175.

[30] S. Mukai. Semi-homogeneous vector bundles on an Abelian variety // J. Math. Kyoto Univ. 1978. V. 18. № 2. P. 239-272.

[31] S. Mukai. On the moduli space of bundles on a K3 surface. I. Vector bundles on algebraic varieties // Tata Inst. Fund. Res. Stud. Math. 1987. V. 11. P. 341-413.

[32] D. Mumford. Abelian Varieties. London: Oxford Univ. Press, 1974. (Tata Inst. Fund. Res. Stud. Math. V. 5.)

[33] В.В.Никулин. Целочисленные симметрические билинейные формы и некоторые их геометрические приложения // Изв. АН СССР. Сер. матем. 1979. Т. 43. № 1. С. 111-177.

[34] Д. О. Орлов. Проективные расслоения, моноидальные преобразования и производные категории когерентных пучков // Изв. РАН. Сер. матем. 1992. Т. 56. № 4. С. 852-862.

[35] D. Orlov. Equivalences of derived categories and K3 surfaces // J. Math. Sci. (New York). 1997. V. 85. № 5. P. 1361-1381.

[36] Д. О. Орлов. Квазикогерентные пучки в коммутативной и некоммутативной геометрии // Изв. РАН. Сер. матем. 2003. Т. 67. № 3. С. 119-138. 
[37] Д. О. Орлов. Производные категории когерентных пучков на абелевых многообразиях и эквивалентности между ними // Изв. РАН. Сер. матем. 2002. Т. 66. № 3. С. 131-158.

[38] A. Polishchuk. Symplectic biextensions and a generalization of the Fourier-Mukai transform // Math. Res. Lett. 1996. V. 3. №6. P. 813-828.

[39] И.И. Пятецкий-Шапиро, И.Р. Шаффаревич. Теорема Торелли для алгебраических поверхностей типа К3 // Изв. АН СССР. Сер. матем. 1971. Т. 35. № 3. С. 530-572.

[40] R. G. Swan. Hochschild cohomology of quasiprojective schemes // J. Pure Appl. Algebra. 1996. V. 110. № 1. P. 57-80.

[41] Théorie des intersections et théorème de Riemann-Roch. Séminaire de Géométrie Algebrique du Bois-Marie 1966-1967 (SGA 6). Dirigé par P. Berthelot, A. Grothendieck, L. Illusie. Alec la collaboration de D. Ferrand, J. P. Jouanolou, O. Jussila, S. Kleiman, M. Raynaud et J. P. Serre. Berlin: Springer-Verlag, 1971. (Lecture Notes in Math. V. 225.)

[42] Théorie des Topos et Cohomologie Étale des Schémas. Séminaire de Géométrie Algebrique du Bois-Marie 1963-1964 (SGA 4). Dirigé par M. Artin, A. Grothendieck et J. L. Verdier. Avec la collaboration de N. Bourbaki, P. Deligne et B. Saint-Donat. V. 1-3. Berlin: Springer-Verlag, 1972-1973. (Lecture Notes in Math. V. 269, 270, 305.)

[43] R. Thomason, T. Trobaugh. Higher algebraic K-theory of schemes and of derived categories // The Grothendieck Festschrift. V. III. Boston: Birkhäuser, 1990. P. 247-435. (Progr. Math. V. 88.)

[44] J. L. Verdier. Categories dérivées // Séminaire de Géométrie Algebrique du Bois-Marie (SGA 4 $\frac{1}{2}$ ). Berlin: Springer-Verlag, 1977. P. 262-311. (Lecture Notes in Math. V. 569.)

Математический институт

Поступила в редакцию

им. В.А. Стеклова РАН

05.02 .2003

E-mail: orlov@mi.ras.ru 